\title{
On the lifting of hermitian modular forms
}

\author{
Tamotsu Ikeda
}

To my parents

\begin{abstract}
Let $K$ be an imaginary quadratic field with discriminant $-D$. We denote by $\mathcal{O}$ the ring of integers of $K$. Let $\chi$ be the primitive Dirichlet character corresponding to $K / \mathbb{Q}$. Let $\Gamma_{K}^{(m)}=\mathrm{U}(m, m)(\mathbb{Q}) \cap \mathrm{GL}_{2 m}(\mathcal{O})$ be the hermitian modular group of degree $m$. We construct a lifting from $S_{2 k}\left(\mathrm{SL}_{2}(\mathbb{Z})\right)$ to $S_{2 k+2 n}\left(\Gamma_{K}^{(2 n+1)}\right.$, $\left.\operatorname{det}^{-k-n}\right)$ and a lifting from $S_{2 k+1}\left(\Gamma_{0}(D), \chi\right)$ to $S_{2 k+2 n}\left(\Gamma_{K}^{(2 n)}\right.$, det $\left.^{-k-n}\right)$. We give an explicit Fourier coefficient formula of the lifting. This is a generalization of the Maass lift considered by Kojima, Krieg and Sugano. We also discuss its extension to the adele group of $\mathrm{U}(m, m)$.
\end{abstract}

\section{Introduction}

In this paper, we are going to discuss a lifting of elliptic cusp forms to hermitian modular forms. This is a hermitian modular analogue of the lifting constructed in [Ike01]. In [Ike01], the author constructed a Siegel cusp form whose Fourier coefficients are closely related to the Fourier coefficients of Eisenstein series of Siegel type.

Let us describe our results. Let $K=\mathbb{Q}\left(\sqrt{-D_{K}}\right)$ be an imaginary quadratic field with discriminant $-D_{K}$. We denote the ring of integers of $K$ by $\mathcal{O}$. The primitive Dirichlet character corresponding to $K / \mathbb{Q}$ is denoted by $\chi$. The hermitian modular group $\Gamma_{K}^{(m)}=\mathrm{U}(m, m)(\mathbb{Q}) \cap \mathrm{GL}_{2 m}(\mathcal{O})$ is the group of all elements

$$
\left(\begin{array}{ll}
A & B \\
C & D
\end{array}\right) \in \mathrm{GL}_{2 m}(\mathcal{O})
$$

such that

$$
A^{\mathrm{t}} \bar{B}=B^{\mathrm{t}} \bar{A}, \quad C^{\mathrm{t}} \bar{D}=D^{\mathrm{t}} \bar{C}, \quad A^{\mathrm{t}} \bar{D}-B^{\mathrm{t}} \bar{C}=\mathbf{1}_{m} .
$$

We let $\Gamma_{K, \infty}^{(m)}$ be the subset of elements

$$
\left(\begin{array}{ll}
A & B \\
C & D
\end{array}\right) \in \Gamma_{K}^{(m)}
$$

such that $C=0$. The hermitian upper half space of degree $m$ is defined by

$$
\mathcal{H}_{m}=\left\{Z \in M_{m}(\mathbb{C}) \mid \frac{1}{2 \sqrt{-1}}\left(Z-{ }^{\mathrm{t}} \bar{Z}\right)>0\right\} .
$$

For

$$
g=\left(\begin{array}{ll}
A & B \\
C & D
\end{array}\right) \in \mathrm{U}(m, m)(\mathbb{R})
$$

Received 22 May 2007, accepted in final form 9 January 2008. 2000 Mathematics Subject Classification 11F55, 11F30.

Keywords: hermitian modular forms, Maass lift.

This journal is (C) Foundation Compositio Mathematica 2008. 


\section{T. IKEDA}

and $Z \in \mathcal{H}_{m}$, we put

$$
g\langle Z\rangle=(A Z+B)(C Z+D)^{-1}, \quad j(g, Z)=\operatorname{det}(C Z+D) .
$$

Let $\sigma$ be a character of $\Gamma_{K}^{(m)}$, which is trivial on

$$
\left\{\left(\begin{array}{cc}
\mathbf{1}_{m} & B \\
0 & \mathbf{1}_{m}
\end{array}\right) \in \Gamma_{K}^{(m)}\right\} \text {. }
$$

A holomorphic function $F$ on $\mathcal{H}_{m}(m \geqslant 2)$ is called a hermitian modular form of weight $l$ with character $\sigma$ if $F(g\langle Z\rangle)=\sigma(g) F(Z) j(g, Z)^{l}$ for any $g \in \Gamma_{K}^{(m)}$.

Recall that a semi-integral hermitian matrix is a hermitian matrix $H \in\left(\sqrt{-D_{K}}\right)^{-1} \mathrm{M}_{m}(\mathcal{O})$ whose diagonal entries are integral. We denote the set of semi-integral hermitian matrices by $\Lambda_{m}(\mathcal{O})$. The set of positive definite elements of $\Lambda_{m}(\mathcal{O})$ is denoted by $\Lambda_{m}(\mathcal{O})^{+}$. For $H \in \Lambda_{m}(\mathcal{O})$, we put $\gamma(H)=\left(-D_{K}\right)^{[m / 2]} \operatorname{det} H$. Note that $\gamma(H) \in \mathbb{Z}$. A hermitian modular form $F$ is called a cusp form if it has a Fourier expansion of the form

$$
F(Z)=\sum_{H \in \Lambda_{m}(\mathcal{O})^{+}} A(H) \exp (2 \pi \sqrt{-1} \operatorname{tr}(H Z)) .
$$

We denote the space of cusp forms of weight $l$ with character $\sigma$ by $S_{l}\left(\Gamma_{K}^{(m)}, \sigma\right)$.

The Eisenstein series $E_{2 l}^{(m)}(Z)$ of weight $2 l$ with character $\operatorname{det}^{-l}$ is defined by

$$
E_{2 l}^{(m)}(Z)=\sum_{g \in \Gamma_{K, \infty}^{(m)} \backslash \Gamma_{K}^{(m)}}(\operatorname{det} g)^{l} j(g, Z)^{-2 l}
$$

This is absolutely convergent for $l>m$. We define the normalized Eisenstein series $\mathcal{E}_{2 l}^{(m)}(Z)$ by

$$
\mathcal{E}_{2 l}^{(m)}(Z)=2^{-m} \prod_{i=1}^{m} L\left(i-2 l, \chi^{i-1}\right) \cdot E_{2 l}^{(m)}(Z) .
$$

If $H \in \Lambda_{m}(\mathcal{O})^{+}$, then the $H$ th Fourier coefficient of $\mathcal{E}_{2 l}^{(m)}(Z)$ is equal to (see $\S 4$ )

$$
|\gamma(H)|^{l-(m / 2)} \prod_{p \mid \gamma(H)} \tilde{F}_{p}\left(H ; p^{-l+(m / 2)}\right) .
$$

Here, $\tilde{F}_{p}(H ; X)$ is a certain Laurent polynomial arising from the Siegel series for $H$.

Then our main theorem can be stated as follows. For simplicity, we assume that $m=2 n$ is even. Let

$$
f(\tau)=\sum_{N=1}^{\infty} a(N) q^{N} \in S_{2 k+1}\left(\Gamma_{0}\left(D_{K}\right), \chi\right)
$$

be a primitive form, whose $L$-function is given by

$$
L(f, s)=\prod_{p \nmid D_{K}}\left(1-a(p) p^{-s}+\chi(p) p^{2 k-2 s}\right)^{-1} \prod_{p \mid D_{K}}\left(1-a(p) p^{-s}\right)^{-1} .
$$

For each prime $p \nmid D_{K}$, we define the Satake parameter $\left\{\alpha_{p}, \beta_{p}\right\}=\left\{\alpha_{p}, \chi(p) \alpha_{p}^{-1}\right\}$ by

$$
\left(1-a(p) X+\chi(p) p^{2 k} X^{2}\right)=\left(1-p^{k} \alpha_{p} X\right)\left(1-p^{k} \beta_{p} X\right) .
$$




\section{LIFTING OF HERMITIAN MODULAR FORMS}

For $p \mid D_{K}$, we put $\alpha_{p}=p^{-k} a(p)$. Put

$$
\begin{aligned}
& A(H)=|\gamma(H)|^{k} \prod_{p \mid \gamma(H)} \tilde{F}_{p}\left(H ; \alpha_{p}\right), \quad H \in \Lambda_{2 n}(\mathcal{O})^{+}, \\
& F(Z)=\sum_{H \in \Lambda_{2 n}(\mathcal{O})^{+}} A(H) \exp (2 \pi \sqrt{-1} \operatorname{tr}(H Z)), \quad Z \in \mathcal{H}_{2 n} .
\end{aligned}
$$

Then our first main theorem in the even case is as follows.

Theorem 5.1. Assume that $m=2 n$ is even. Let $f(\tau), A(H)$ and $F(Z)$ be as above. Then we have $F \in S_{2 k+2 n}\left(\Gamma_{K}^{(2 n)}, \operatorname{det}^{-k-n}\right)$.

(In the case when $m$ is odd, we consider a similar lifting for a normalized Hecke eigenform $f \in S_{2 k}\left(\mathrm{SL}_{2}(\mathbb{Z})\right)$; see Theorem 5.2.) To prove this theorem, we use the theory of a compatible family of Eisenstein series as in [Ike01]. The theory of a compatible family of Eisenstein series is a method to prove that a certain Fourier series is a modular form of one variable. The Fourier series we consider can be regarded as the sum of Whittaker functions, and the behavior of a Whittaker function is determined by Fourier coefficients of Eisenstein series. In [Ike01], the author considered the compatible family of Eisenstein series of half-integral weight. In this paper, we consider the compatible family of Eisenstein series of integral weight. This case is a little more complicated than that of [Ike01] because the automorphic representation of $\mathrm{SL}_{2}(\mathbb{A})$ generated by $f$ may be reducible. Instead of using a Whittaker function on $\mathrm{SL}_{2}(\mathbb{A})$, we extend it to $\mathrm{GL}_{2}(\mathbb{A})$. We have to show that this extension to $\mathrm{GL}_{2}(\mathbb{A})$ is possible in a compatible way for a family of Eisenstein series arising from the Fourier-Jacobi coefficients of $\mathcal{E}_{2 k^{\prime}+2 n}^{(2 n)}$. This problem is treated in $\S \S 8$ and 9 . Using this theory, we prove Theorems 5.1 and 5.2 in $\S 10$.

In $\S \S 12$ and 13 , we prove that the lift $F$ can be extended to an automorphic form on the adele group of the unitary group $\mathrm{U}(m, m)$. The extension Lift ${ }^{(m)}(f)$ is a common Hecke eigenform of all Hecke operators of the unitary group, if it is not identically zero (Theorem 13.6). Moreover, the standard $L$-function $L\left(s\right.$, Lift $^{(2 n)}(f)$, st) is given by (see Theorem 18.1)

$$
\prod_{i=1}^{m} L\left(s+k+n-i+\frac{1}{2}, f\right) L\left(s+k+n-i+\frac{1}{2}, f, \chi\right) .
$$

Following Kohnen [Koh02], we discuss the 'linearization' of the lifting. The case when $m$ is odd is fairly easy, and will be treated in $\S 14$. Assume now that $m=2 n$ is even. Then we reformulate the main theorem in terms of a certain linear map from a subspace $S_{2 k+1}^{*}\left(\Gamma_{0}\left(D_{K}\right), \chi\right) \subset$ $S_{2 k+1}\left(\Gamma_{0}\left(D_{K}\right), \chi\right)$ to $S_{2 k+2 n}\left(\Gamma_{K}^{(2 n)}\right.$, $\left.\operatorname{det}^{-k-n}\right)$. (In fact, we need to consider a certain twisting by an ideal $\mathfrak{c}$ of $K$, but for simplicity we consider the case $\mathfrak{c}=\mathcal{O}$ here.) Decompose the character $\chi$ into a product $\chi=\prod_{q \mid D_{K}} \chi_{q}$, where $\chi_{q}$ is a character whose conductor is a power of a prime $q$. Put

$$
\mathbf{a}_{D_{K}}(N)=\prod_{q \mid D_{K}}\left(1+\chi_{q}\left((-1)^{n} N\right)\right)
$$

Following Krieg [Kri91], we define $S_{2 k+1}^{*}\left(\Gamma_{0}\left(D_{K}\right), \chi\right)$ by the space of cusp forms

$$
f_{0}(\tau)=\sum_{N>0} a_{f_{0}}(N) q^{N} \in S_{2 k+1}\left(\Gamma_{0}\left(D_{K}\right), \chi\right)
$$

such that $a_{f_{0}}(N)=0$ whenever $\mathbf{a}_{D_{K}}(N)=0$. For each primitive form $f \in S_{2 k+1}\left(\Gamma_{0}\left(D_{K}\right), \chi\right)$, we define $f^{*} \in S_{2 k+1}^{*}\left(\Gamma_{0}\left(D_{K}\right), \chi\right)$ as the unique element of $S_{2 k+1}\left(\Gamma_{0}\left(D_{K}\right), \chi\right)$ such that $a_{f^{*}}(N)=$ $\mathbf{a}_{D_{K}}(N) a_{f}(N)$ whenever $\left(N, D_{K}\right)=1$. Then we can show that there exists an injective linear map

$$
\iota: S_{2 k+1}^{*}\left(\Gamma_{0}\left(D_{K}\right), \chi\right) \rightarrow S_{2 k+2 n}\left(\Gamma_{K}^{(2 n)}, \operatorname{det}^{-k-n}\right)
$$




\section{T. IKEDA}

such that $F(Z)$ is equal to $\iota\left(f^{*}\right)$ (Theorem 15.18). It follows that $F=0$ if and only if $f^{*}=0$. It is easy to prove that $f^{*}=0$ if and only if $n$ is odd and $f$ comes from a Hecke character of some imaginary quadratic field (Corollary 15.20). As for the lifting Lift ${ }^{(2 n)}(f)$ to the adele group $\mathrm{U}(2 n, 2 n)(\mathbb{A})$, we show that Lift $^{(2 n)}(f)=0$ if and only if $n$ is odd and $f$ comes from a Hecke character of $K$ (Corollary 15.21).

We discuss the case $m=2$ in $\S 16$. In this case, the theory of the lifting has already been treated by Kojima [Koj82], Gritsenko [Gri90], Krieg [Kri91], Sugano [Sug85], and Klosin [Klo07a, Klo07b]. In $\S 17$, we calculate the Petersson inner product of the hermitian Maass lift in the case $m=2$, by using the results of Sugano [Sug85].

In $\S 18$, we discuss the relation to the Arthur conjecture. The Arthur parameter associated to our lift can be described as follows. We now admit the Arthur conjecture and the existence of the hypothetical Langlands group $\mathcal{L}_{\mathbb{Q}}$. Let $m$ be a positive integer. Recall that the $L$-group of $\mathcal{G}=$ $\mathrm{U}(m, m)$ is a semi-direct product $\mathrm{GL}_{2 m}(\mathbb{C}) \rtimes W_{\mathbb{Q}}$, where $W_{\mathbb{Q}}$ is the Weil group of $\mathbb{Q}$. The canonical homomorphism $\mathcal{L}_{\mathbb{Q}} \rightarrow W_{\mathbb{Q}}$ is denoted by pr. Let $f$ be a primitive form of $S_{2 k+1}\left(\Gamma_{0}\left(D_{K}\right), \chi\right)$ or a normalized Hecke eigenform of $S_{2 k}\left(\mathrm{SL}_{2}(\mathbb{Z})\right)$ according as $m$ is even or odd. Let $\tau$ be an irreducible cuspidal automorphic representation of $\mathrm{GL}_{2}\left(\mathbb{A}_{\mathbb{Q}}\right)$ generated by $f$. Note that the central character $\omega_{\tau}$ is equal to $\chi^{m-1}$. We denote the Langlands parameter of $\tau$ by $\rho_{\tau}: \mathcal{L}_{\mathbb{Q}} \rightarrow \mathrm{GL}_{2}(\mathbb{C})$. Let $\operatorname{Sym}^{m-1}:$ $\mathrm{SL}_{2}(\mathbb{C}) \rightarrow \mathrm{SL}_{m}(\mathbb{C})$ be the $m$-dimensional irreducible representation of $\mathrm{SL}_{2}(\mathbb{C})$. We put

$$
\rho_{\tau}^{(m)}(u)=\left(\begin{array}{ll}
\omega_{\tau}(u) a \cdot \mathbf{1}_{m} & b \cdot \mathbf{1}_{m} \\
\omega_{\tau}(u) c \cdot \mathbf{1}_{m} & d \cdot \mathbf{1}_{m}
\end{array}\right) \rtimes \operatorname{pr}(u)
$$

for $u \in \mathcal{L}_{\mathbb{Q}}$,

$$
\rho_{\tau}(u)=\left(\begin{array}{ll}
a & b \\
c & d
\end{array}\right)
$$

and we put

$$
\rho_{\tau}^{(m)}(x)=\left(\begin{array}{cc}
\operatorname{Sym}^{m-1}(x) & 0 \\
0 & \operatorname{Sym}^{m-1}(x)
\end{array}\right) \rtimes 1
$$

for $x \in \mathrm{SL}_{2}(\mathbb{C})$. Then, $\rho_{\tau}^{(m)}: \mathcal{L}_{\mathbb{Q}} \times \mathrm{SL}_{2}(\mathbb{C}) \rightarrow{ }^{L} \mathcal{G}=\mathrm{GL}_{2 m}(\mathbb{C}) \rtimes W_{\mathbb{Q}}$ should be the Arthur parameter for $\operatorname{Lift}^{(m)}(f)$. By using this $A$-parameter, we will show that our result is compatible with the conjectural Arthur multiplicity formula.

\section{Notation}

Let $K$ be an imaginary quadratic field with discriminant $-D=-D_{K}$. When there is no fear of confusion, we drop the subscript $K$. We denote by $\mathcal{O}=\mathcal{O}_{K}$ the ring of integers of $K$. The number of roots of unity contained in $K$ is denoted by $w_{K}$. The non-trivial automorphism of $K$ is denoted by $x \mapsto \bar{x}$. The primitive Dirichlet character corresponding to $K / \mathbb{Q}$ is denoted by $\chi$. We denote by $\mathcal{O}^{\sharp}=(\sqrt{-D})^{-1} \mathcal{O}$ the inverse different ideal of $K / \mathbb{Q}$. For each prime $p$, we set $K_{p}=K \otimes \mathbb{Q}_{p}$ and $\mathcal{O}_{p}=\mathcal{O} \otimes \mathbb{Z}_{p}$. The sets of hermitian matrices of size $m$ with entries in $K, K_{p}, \mathcal{O}$, and $\mathcal{O}_{p}$ are denoted by $\mathcal{H}_{m}(K), \mathcal{H}_{m}\left(K_{p}\right), \mathcal{H}_{m}(\mathcal{O})$, and $\mathcal{H}_{m}\left(\mathcal{O}_{p}\right)$, respectively. The adele ring of $\mathbb{Q}$ is denoted by $\mathbb{A}_{\mathbb{Q}}$ or $\mathbb{A}$. The finite part of the adele ring is denoted by $\mathbb{A}_{\mathrm{f}}$. Similarly, we denote the adele ring and the finite adele ring of $K$ by $\mathbb{A}_{K}$ and $\mathbb{A}_{K, \mathrm{f}}$, respectively.

Let $\underline{\chi}=\bigotimes_{v} \underline{\chi}_{v}$ be the character of the idele class group $\mathbb{A}^{\times} / \mathbb{Q}^{\times}$determined by $\chi$. Then $\underline{\chi}_{v}$ is the character of $\mathbb{Q}_{v}^{\times}$corresponding to $\mathbb{Q}_{v}(\sqrt{-D}) / \mathbb{Q}_{v}$ and is given by the Hilbert symbol

$$
\underline{\chi}_{v}(t)=\left(\frac{-D, t}{\mathbb{Q}_{v}}\right)
$$

for $t \in \mathbb{Q}_{v}^{\times}$. 


\section{LIFTING OF HERMITIAN MODULAR FORMS}

We set $\mathbf{e}(T)=\exp (2 \pi \sqrt{-1} \operatorname{tr}(T))$ if $T$ is a square matrix with entries in $\mathbb{C}$. When $p$ is a prime, $\mathbf{e}_{p}$ is the unique additive character of $\mathbb{Q}_{p}$ such that $\mathbf{e}_{p}(x)=\exp (-2 \pi \sqrt{-1} x)$ for $x \in \mathbb{Z}\left[p^{-1}\right]$. Note that $\mathbf{e}_{p}$ is of order 0 . We put $\mathbf{e}_{\mathbb{A}}(x)=\mathbf{e}\left(x_{\infty}\right) \prod_{p<\infty} \mathbf{e}_{p}\left(x_{p}\right)$ for an adele $x=\left(x_{v}\right)_{v} \in \mathbb{A}$.

In $\S \S 3$ and $8, F$ will denote a non-archimedean local field. When $\psi$ is an additive character of $F$ and $\rho$ is a quasi-character of $F^{\times}$, the $L$-factor $L(s, \rho)$ and the $\varepsilon$-factor $\varepsilon(s, \rho, \psi)$ are defined as in Tate [Tat79]. We set $\varepsilon^{\prime}(s, \rho, \psi)=\varepsilon(s, \rho, \psi) L\left(1-s, \rho^{-1}, \psi\right) L(s, \rho)^{-1}$.

\section{Unitary groups and hermitian modular forms}

We recall some basic facts about hermitian modular forms (cf. Braun [Bra51]). The unitary group $\mathcal{G}^{(m)}=\mathrm{U}(m, m)$ is an algebraic group defined over $\mathbb{Q}$, whose group of $R$-valued points is given by

$$
\left\{g \in \mathrm{GL}_{2 m}(R \otimes K) \mid g\left(\begin{array}{cc}
0 & -\mathbf{1}_{m} \\
\mathbf{1}_{m} & 0
\end{array}\right) \mathrm{t} \bar{g}=\left(\begin{array}{cc}
0 & -\mathbf{1}_{m} \\
\mathbf{1}_{m} & 0
\end{array}\right)\right\}
$$

for any $\mathbb{Q}$-algebra $R$. When there is no fear of confusion, we drop the superscript $(m)$. We define the hermitian modular group by $\Gamma_{K}^{(m)}=\mathcal{G}^{(m)}(\mathbb{Q}) \cap \mathrm{GL}_{2 m}(\mathcal{O})$. Put

$$
\Gamma_{K, \infty}^{(m)}=\left\{\left(\begin{array}{ll}
A & B \\
C & D
\end{array}\right) \in \Gamma_{K}^{(m)} \mid C=0\right\} .
$$

Note that $\Gamma_{K}^{(1)}=\mathrm{SL}_{2}(\mathbb{Z}) \cdot\left\{\alpha \cdot \mathbf{1}_{2} \mid \alpha \in \mathcal{O}^{\times}\right\}$. A hermitian matrix $H \in \mathcal{H}_{m}(K)$ is semi-integral if $\operatorname{tr}(H R) \in \mathbb{Z}$ for any $R \in \mathcal{H}_{m}(\mathcal{O})$. Note that $H \in \mathcal{H}_{m}(K)$ is semi-integral if and only if the diagonal entries of $H$ are integral and $\sqrt{-D_{K}} \cdot H \in \mathrm{M}_{m}(\mathcal{O})$. We denote the set of semi-integral hermitian matrices of size $m$ by $\Lambda_{m}(\mathcal{O})$. Similarly, we define $\Lambda_{m}\left(\mathcal{O}_{p}\right)$. Then we have $\Lambda_{m}\left(\mathcal{O}_{p}\right)=\Lambda(\mathcal{O}) \otimes_{\mathbb{Z}} \mathbb{Z}_{p}$. The subset of $\Lambda_{m}(\mathcal{O})$ consisting of all positive definite elements is denoted by $\Lambda_{m}(\mathcal{O})^{+}$.

The hermitian upper half space $\mathcal{H}_{m}$ is defined by

$$
\mathcal{H}_{m}=\left\{Z \in M_{m}(\mathbb{C}) \mid \frac{1}{2 \sqrt{-1}}\left(Z-{ }^{\mathrm{t}} \bar{Z}\right)>0\right\} .
$$

Note that $\mathcal{H}_{1}=\mathfrak{H}_{1}$ is the usual upper half plane. Then the unitary group $\mathcal{G}^{(m)}(\mathbb{R})$ acts on $\mathcal{H}_{m}$ by

$$
g\langle Z\rangle=(A Z+B)(C Z+D)^{-1}, \quad Z \in \mathcal{H}_{m}, g=\left(\begin{array}{ll}
A & B \\
C & D
\end{array}\right) \in \mathcal{G}^{(m)}(\mathbb{R}) .
$$

We put $j(g, Z)=\operatorname{det}(C Z+D)$ for $Z \in \mathcal{H}_{m}$ and

$$
g=\left(\begin{array}{ll}
A & B \\
C & D
\end{array}\right) \in \mathcal{G}^{(m)}(\mathbb{R})
$$

If $F(Z)$ is a function on $\mathcal{H}_{m}$, we put

$$
\left(\left.F\right|_{l} g\right)(Z)=F(g\langle Z\rangle) j(g, Z)^{-l}
$$

for $g \in \mathcal{G}^{(m)}(\mathbb{R})$. When $l$ is clear from the context, we sometimes drop it. Let $\sigma$ be a character of $\Gamma_{K}^{(m)}$, which is trivial on

$$
\left\{\left(\begin{array}{cc}
\mathbf{1}_{m} & B \\
0 & \mathbf{1}_{m}
\end{array}\right) \in \Gamma_{K}^{(m)}\right\} .
$$

A holomorphic function $F$ on $\mathcal{H}_{m}$ is called a hermitian modular form of weight $l$ with character $\sigma$ if $\left.F\right|_{l} g=\sigma(g) F$ for any $g \in \Gamma_{K}^{(m)}$. When $m=1$, the usual holomorphy condition at the cusp is required. A hermitian modular form $F(Z)$ has a Fourier expansion of the form

$$
F(Z)=\sum_{\substack{H \in \Lambda_{m}(\mathcal{O}) \\ H \geqslant 0}} A(H) \mathbf{e}(H Z)
$$




\section{T. IKEDA}

Here, $H \geqslant 0$ means that the hermitian matrix $H$ is positive semi-definite. A hermitian modular form $F$ is called a cusp form if the Fourier coefficients $A(H)$ vanish unless $H \in \Lambda(\mathcal{O})^{+}$. The space of hermitian modular forms (respectively hermitian cusp forms) of weight $l$ with character $\sigma$ is denoted by $M_{l}\left(\Gamma_{K}^{(m)}, \sigma\right)$ (respectively $S_{l}\left(\Gamma_{K}^{(m)}, \sigma\right)$ ).

\section{Siegel series for unitary groups}

In this section, we consider Siegel series associated to non-degenerate semi-integral hermitian matrices. Fix a prime $p$. Let $\mathbf{e}_{p}$ be the unique additive character of $\mathbb{Q}_{p}$ such that $\mathbf{e}_{p}(x)=\exp (-2 \pi \sqrt{-1} x)$ for $x \in \mathbb{Z}\left[p^{-1}\right]$. Note that $\mathbf{e}_{p}$ is of order 0 . Put $\xi_{p}=\chi(p)$, i.e.

$$
\xi_{p}= \begin{cases}1 & \text { if } K_{p} \simeq \mathbb{Q}_{p} \oplus \mathbb{Q}_{p}, \\ -1 & \text { if } K_{p} / \mathbb{Q}_{p} \text { is an unramified quadratic extension } \\ 0 & \text { if } K_{p} / \mathbb{Q}_{p} \text { is a ramified quadratic extension }\end{cases}
$$

For $H \in \Lambda_{m}\left(\mathcal{O}_{p}\right)$, det $H \neq 0$, we put

$$
\gamma(H)=\left(-D_{K}\right)^{[m / 2]} \operatorname{det} H .
$$

It is easily seen that $\gamma(H) \in \mathbb{Z}_{p}$. Similarly, we have $\gamma(H) \in \mathbb{Z}$ for $H \in \Lambda_{m}(\mathcal{O})$.

The Siegel series for $H$ is defined by

$$
b_{p}(H, s)=\sum_{R \in \mathcal{H}_{m}\left(K_{p}\right) / \mathcal{H}_{m}\left(\mathcal{O}_{p}\right)} \mathbf{e}_{p}(\operatorname{tr}(H R)) p^{-\operatorname{ord}_{p}(\nu(R)) s}, \quad \operatorname{Re}(s) \gg 0 .
$$

The ideal $\nu(R) \subset \mathbb{Z}_{p}$ is defined as follows. Choose an element

$$
\left(\begin{array}{ll}
A & B \\
C & D
\end{array}\right) \in \mathcal{G}^{(m)}\left(\mathbb{Q}_{p}\right) \cap \mathrm{SL}_{2 m}\left(\mathcal{O}_{p}\right)
$$

such that $\operatorname{det} D \neq 0, D^{-1} C=R$. Then $\nu(R)=(\operatorname{det} D) \mathbb{Z}_{p}$. Note that $\operatorname{det} D \in \mathbb{Q}_{p}$.

We define a polynomial $t_{p}(K / \mathbb{Q} ; X) \in \mathbb{Z}[X]$ by

$$
t_{p}(K / \mathbb{Q} ; X)=\prod_{i=1}^{[(m+1) / 2]}\left(1-p^{2 i} X\right) \prod_{i=1}^{[m / 2]}\left(1-p^{2 i-1} \xi_{p} X\right)
$$

There exists a polynomial $F_{p}(H ; X) \in \mathbb{Z}[X]$ with constant term 1 such that

$$
F_{p}\left(H ; p^{-s}\right)=b_{p}(H, s) t_{p}\left(K / \mathbb{Q} ; p^{-s}\right)^{-1} \text {. }
$$

For a proof of this fact, see [Shi97]. Clearly, $F_{p}\left({ }^{\mathrm{t}} \bar{A} H A ; X\right)=F_{p}(H ; X)$ for any $A \in \mathrm{GL}_{m}\left(\mathcal{O}_{p}\right)$. Moreover, $F_{p}(H ; X)$ satisfies the functional equation

$$
F_{p}\left(H ; p^{-2 m} X^{-1}\right)=\underline{\chi}_{p}(\gamma(H))^{m-1}\left(p^{m} X\right)^{-\operatorname{ord}_{p} \gamma(H)} F_{p}(H ; X),
$$

which follows from the results of Kudla and Sweet [KS97]. We will discuss it in $\S 3$.

The functional equation implies that $\operatorname{deg} F_{p}(H ; X)=\operatorname{ord}_{p} \gamma(H)$. In particular, if $p \nmid \gamma(H)$, then $F_{p}(H ; X)=1$.

Definition 2.1. For $H \in \Lambda_{m}\left(\mathcal{O}_{p}\right)$, $\operatorname{det} H \neq 0$, we put

$$
\tilde{F}_{p}(H ; X)=X^{\operatorname{ord}_{p} \gamma(H)} F_{p}\left(H ; p^{-m} X^{-2}\right) .
$$

Note that the highest term and the lowest term of $\tilde{F}_{p}(H ; X)$ are $X^{\operatorname{ord}_{p} \gamma(H)}$ and $\underline{\chi}_{p}(\gamma(H))^{m-1}$ $X^{-\operatorname{ord}_{p} \gamma(H)}$, respectively. The following lemma follows immediately from the functional equation of $F_{p}(H ; X)$. 


\section{LIFTING OF HERMITIAN MODULAR FORMS}

Lemma 2.2. For $H \in \Lambda_{m}\left(\mathcal{O}_{p}\right)$, $\operatorname{det} H \neq 0$, we have:

$$
\begin{aligned}
\tilde{F}_{p}\left(H ; X^{-1}\right) & =\tilde{F}_{p}(H ; X) & & \text { if } m \text { is odd, } \\
\tilde{F}_{p}\left(H ; X^{-1}\right) & =\underline{\chi}_{p}(\gamma(H)) \tilde{F}_{p}(H ; X) & & \text { if } m \text { is even, } \\
\tilde{F}_{p}\left(H ; \xi_{p} X^{-1}\right) & =\tilde{F}_{p}(H ; X) & & \text { if } m \text { is even and } p \nmid D_{K} .
\end{aligned}
$$

We will need the following lemma later.

LEMma 2.3. There exists a constant $M>0$ such that

$$
\left|\tilde{F}_{p}(H ; \omega)\right| \leqslant p^{M \cdot \operatorname{ord}_{p}(\gamma(H))}
$$

for any $H \in \Lambda_{m}\left(\mathcal{O}_{p}\right)$, $\operatorname{det} H \neq 0$ and any $\omega \in \mathbb{C},|\omega|=1$. The constant $M$ does not depend on $p$. Proof. We may assume that $p \mid \gamma(H)$. Put $d=\operatorname{ord}_{p}(\gamma(H))=\operatorname{deg} F_{p}(H ; X)$. For each

$$
\varphi(X)=\sum_{N=0}^{\infty} a_{N} X^{N} \in \mathbb{C}[[X]],
$$

we put $H_{d}(\varphi)=\max \left(\left|a_{0}\right|, \ldots,\left|a_{d}\right|\right)$. Then

$$
H_{d}\left(\varphi_{1} \varphi_{2}\right) \leqslant(d+1) H_{d}\left(\varphi_{1}\right) H_{d}\left(\varphi_{2}\right)
$$

for $\varphi_{1}, \varphi_{2} \in \mathbb{C}[[X]]$. For each positive integer $l$, we define a formal power series $\alpha_{H}^{l}(t)$ such that

$$
\alpha_{H}^{l}\left(p^{-s}\right)=\sum_{R \in p^{-l} \Lambda_{m}\left(\mathcal{O}_{p}\right) / \Lambda_{m}\left(\mathcal{O}_{p}\right)} \mathbf{e}_{p}(\operatorname{tr}(H R)) p^{-\operatorname{ord}_{p}(\nu(R)) s} .
$$

Then it has been proved by Shimura [Shi97] that, if $l \geqslant 2 d+1$, then $\alpha_{H}^{l}\left(p^{-s}\right)=b_{p}(H, s)$. Since $b_{p}(H, s)=t_{p}\left(K / \mathbb{Q} ; p^{-s}\right) F_{p}\left(H ; p^{-s}\right)$, we have

$$
H_{d}\left(t_{p}(K / \mathbb{Q} ; X) F_{p}(H ; X)\right) \leqslant p^{(2 d+1) m^{2}} \leqslant p^{3 d m^{2}} .
$$

On the other hand, we also have

$$
\begin{aligned}
H_{d}\left(t_{p}(K / \mathbb{Q} ; X)^{-1}\right) & \leqslant H_{d}\left(\prod_{i=1}^{m}\left(1-p^{i} X\right)^{-1}\right) \\
& \leqslant(d+1)^{m-1} \prod_{i=1}^{m} H_{d}\left(\left(1-p^{i} X\right)^{-1}\right) \\
& \leqslant(d+1)^{m-1} p^{d m(m+1) / 2} .
\end{aligned}
$$

By the obvious estimate $(d+1) \leqslant p^{d}$, we have

$$
H_{d}\left(F_{p}(H ; X)\right) \leqslant(d+1)^{m} p^{d m(m+1) / 2} p^{3 d m^{2}} \leqslant p^{d\left(7 m^{2}+3 m\right) / 2} .
$$

Since $|\omega|=1$, we have

$$
\left|\tilde{F}_{p}(H ; \omega)\right|=\left|F_{p}\left(H ; p^{-m} \omega^{-2}\right)\right| \leqslant(d+1) p^{d\left(7 m^{2}+3 m\right) / 2} \leqslant p^{d\left(7 m^{2}+3 m+2\right) / 2} .
$$

It follows that $\left|\tilde{F}_{p}(H ; \omega)\right| \leqslant p^{d\left(7 m^{2}+3 m+2\right) / 2}$.

\section{A proof of the functional equation}

In this section, $F=F_{v}$ is a non-archimedean local field. We denote the ring of integers, the absolute value, and the order of the residue field by $\mathfrak{o},||$ and $q$, respectively. Let $E$ be either a quadratic extension of $F$ or $F \oplus F$. We denote the character corresponding to $E / F$ by $\underline{\chi}$. We denote the 


\section{T. IKEDA}

discriminant ideal of $E / F$ by $\mathfrak{D}$. Put $G=\mathrm{SU}(m, m)_{E / F}$. For a quasi-character $\rho: F^{\times} \rightarrow \mathbb{C}^{\times}$, we denote the degenerate principal series $\operatorname{Ind}_{P}^{G}\left(\rho \cdot||^{s}\right)$ by $I(\rho, s)$. Here $P$ is the Siegel parabolic subgroup of $G$. The space of $I(\rho, s)$ consists of all locally constant functions $\Phi(g)$ on $G$ such that

$$
\Phi\left(\left(\begin{array}{cc}
A & B \\
0 & \mathrm{t} \\
\bar{A}^{-1}
\end{array}\right) g\right)=|\operatorname{det} A|^{s+m} \Phi(g)
$$

for any

$$
\left(\begin{array}{cc}
A & B \\
0 & { }^{\mathrm{t}} \bar{A}^{-1}
\end{array}\right) \in P
$$

and any $g \in G$.

Fix an additive character $\psi$ of $F$. For $\Phi(g) \in I(\rho, s)$ and a non-degenerate hermitian matrix $H \in \mathcal{H}_{m}(E)$, put

$$
\begin{aligned}
M(s, \rho) \Phi(g) & =\int_{\mathcal{H}_{m}(E)} \Phi\left(w\left(\begin{array}{cc}
\mathbf{1}_{m} & x \\
0 & \mathbf{1}_{m}
\end{array}\right) g, s\right) d x \\
\mathrm{Wh}_{H}(s) \Phi(g) & =\int_{\mathcal{H}_{m}(E)} \Phi\left(w\left(\begin{array}{cc}
\mathbf{1}_{m} & x \\
0 & \mathbf{1}_{m}
\end{array}\right) g, s\right) \overline{\psi(\operatorname{tr} H x)} d x .
\end{aligned}
$$

Here, the Haar measure $d x$ is the self-dual measure for the pairing $(x, y) \mapsto \psi(\operatorname{tr}(x y))$. These integrals are absolutely convergent for $\operatorname{Re}(s) \gg 0$ and can be meromorphically continued to the whole complex plane. If $s$ is not a pole of $M(s, \rho)$, then $M(s, \rho) \Phi(g) \in I\left(\rho^{-1},-s\right)$. Moreover, it is known that $\mathrm{Wh}_{H}(s)$ is entire.

Proposition 3.1 (Kudla and Sweet). The following functional equation holds:

$$
\mathrm{Wh}_{H}(-s) \circ M(s, \rho)=\kappa_{H}(s, \rho, \psi) \mathrm{Wh}_{H}(s),
$$

where

$$
\kappa_{H}(s, \rho, \psi)=\rho(\operatorname{det} H)^{-1}|\operatorname{det} H|^{-s} \gamma(E / F, \psi)^{m(m-1) / 2} \underline{\chi}(\operatorname{det} H)^{m-1} \prod_{r=1}^{m} \varepsilon^{\prime}\left(s-m+r, \rho \underline{\chi}^{r-1}, \psi\right)^{-1} .
$$

Here $\gamma(E / F, \psi)$ is the Weil factor for $E / F$ with respect to $\psi$, and $\varepsilon^{\prime}(s, \rho, \psi)=\varepsilon(s, \rho, \psi) L(1-$ $\left.s, \rho^{-1}\right) L(s, \rho)^{-1}$. This proposition is Proposition 3.1 of Kudla and Sweet [KS97] when $E$ is a quadratic extension of $F$. When $E=F \oplus F$, see [KS97, p. 303].

We assume that $\psi$ has order 0 and $\rho=\mathbf{1}$ is the trivial character. Let $\Phi_{0}^{(s)} \in I(s, \mathbf{1})$ be the unique element such that $\Phi_{0}(g)=1$ for $g \in G \cap \mathrm{SL}_{2 m}(\mathfrak{o})$. Then $\mathrm{Wh}_{H}(s) \Phi_{0}^{(s)}\left(\mathbf{1}_{2 m}\right)$ is an analogue of the Siegel series considered in the last section. It is known (cf. Shimura [Shi97]) that there exists a polynomial $F_{v}(H ; X) \in \mathbb{Z}[X]$ such that $\mathrm{Wh}_{H}(s) \Phi_{0}^{(s)}\left(\mathbf{1}_{2 m}\right)$ is equal to

$$
|\mathfrak{D}|^{m(m-1) / 4} \prod_{r=1}^{m} \frac{1}{L\left(s+m+1-r, \underline{\chi}^{r-1}\right)} \cdot F_{v}\left(H ; q^{-s-m}\right) .
$$

By standard Gindikin-Karpelevich argument, we have

$$
M(s, 1) \Phi_{0}^{(s)}=|\mathfrak{D}|^{m(m-1) / 2} \prod_{r=1}^{m} \frac{L\left(s-m+r, \underline{\chi}^{r-1}\right)}{L\left(s+m+1-r, \underline{\chi}^{r-1}\right)} \Phi_{0}^{(-s)} .
$$


It follows that

$$
\begin{aligned}
\mathrm{Wh}_{H}(-s) \circ M(s, 1) \Phi_{0}^{(s)}\left(\mathbf{1}_{2 m}\right)= & |\mathfrak{D}|^{m(m-1) / 2} \prod_{r=1}^{m} \frac{L\left(s-m+r, \underline{\chi}^{r-1}\right)}{L\left(s+m+1-r, \underline{\chi}^{r-1}\right)} \\
& \times \prod_{r=1}^{m} \frac{1}{L\left(-s+m-r+1, \underline{\chi}^{r-1}\right)} \cdot F_{v}\left(H ; q^{s-m}\right) .
\end{aligned}
$$

By Proposition 3.1, we have

$$
\begin{aligned}
& |\operatorname{det} H|^{-s} \gamma(E / F, \psi)^{m(m-1) / 2} \underline{\chi}(\operatorname{det} H)^{m-1} \prod_{r=1}^{m} \varepsilon\left(s-m+r, \underline{\chi}^{r-1}, \psi\right)^{-1} \cdot F_{v}\left(H ; q^{-s-m}\right) \\
& \quad=|\mathfrak{D}|^{m(m-1) / 4} F_{v}\left(H ; q^{s-m}\right) .
\end{aligned}
$$

Since $\varepsilon(s, \underline{\chi}, \psi)=\gamma(E / F, \psi)^{-1}|\mathfrak{D}|^{s-(1 / 2)}$ and $\gamma(E / F, \psi)^{2}=\underline{\chi}(-1)$, we obtain the following functional equation for $F_{v}(H ; X)$.

Corollary 3.2. The polynomial $F_{v}(H ; X)$ satisfies the following functional equation:

(i) if $m=2 n$,

$$
F_{v}\left(H ; q^{-2 m} X^{-1}\right)=\underline{\chi}\left((-1)^{n} \operatorname{det} H\right)\left(q^{m} X\right)^{-\operatorname{ord}\left(\mathfrak{D}^{n} \operatorname{det} H\right)} F_{v}(H ; X) ;
$$

(ii) if $m=2 n+1$,

$$
F_{v}\left(H ; q^{-2 m} X^{-1}\right)=\left(q^{m} X\right)^{-\operatorname{ord}\left(\mathfrak{D}^{n} \operatorname{det} H\right)} F_{v}(H ; X)
$$

Remark 3.3. When $E / F=K_{p} / \mathbb{Q}_{p}$, we obtain the functional equation of $F_{p}(H ; X)$. Note that $\underline{\chi}_{p}(-1)=\underline{\chi}_{p}(-D)$, since

$$
\underline{\chi}_{p}(D)=\left(\frac{-D, D}{\mathbb{Q}_{p}}\right)=1
$$

\section{Fourier coefficients of hermitian Eisenstein series}

For simplicity, we assume that $l$ is a sufficiently large integer. Let $E_{2 l}^{(m)}(Z, s)$ be the Eisenstein series of weight $2 l$ on the hermitian upper half space $\mathcal{H}_{m}$. For $Z \in \mathcal{H}_{m}$, we put $X=\left(Z+{ }^{\mathrm{t}} \bar{Z}\right) / 2$ and $Y=\left(Z-{ }^{\mathrm{t}} \bar{Z}\right) /(2 \sqrt{-1})$. Then the hermitian Eisenstein series $E_{2 l}^{(m)}(Z, s)$ is defined by

$$
E_{2 l}^{(m)}(Z, s)=(\operatorname{det} Y)^{s-l} \sum_{g \in \Gamma_{K, \infty}^{(m)} \backslash \Gamma_{K}^{(m)}}(\operatorname{det} g)^{l} j(g, Z)^{-2 l}|j(g, Z)|^{-2 s+2 l} .
$$

The series $E_{2 l}^{(m)}(Z, s)$ is absolutely convergent for $\operatorname{Re}(s)>m$ and

$$
\left.E_{2 l}^{(m)}(*, s)\right|_{2 l} g=(\operatorname{det} g)^{-l} E_{2 l}^{(m)}(*, s)
$$

for any $g \in \Gamma_{K}^{(m)}$. Then the Fourier expansion of $E_{2 l}^{(m)}(Z, s)$ is

$$
E_{2 l}^{(m)}(Z, s)=\sum_{H \in \Lambda_{m}(\mathcal{O})} c_{2 l}^{(m)}(H ; Y, s) \mathbf{e}(H X)
$$




\section{T. IKEDA}

If $H$ is non-degenerate, we have

$$
\begin{aligned}
c_{2 l}^{(m)}(H ; Y, s) & =\left(\frac{D}{4}\right)^{-m(m-1) / 4}(\operatorname{det} Y)^{s-l} \Xi(Y, H ; s+l, s-l) \prod_{p} b_{p}\left(H, p^{-2 s}\right) \\
& =\left(\frac{D}{4}\right)^{-m(m-1) / 4}(\operatorname{det} Y)^{s-l} \frac{\Xi(Y, H ; s+l, s-l)}{\prod_{i=1}^{m} L\left(2 s+1-i, \chi^{i-1}\right)} \prod_{p \mid \gamma(H)} F_{p}\left(H ; p^{-2 s}\right) .
\end{aligned}
$$

Here (see Shimura [Shi97]),

$$
\Xi\left(g, h ; s, s^{\prime}\right)=\int_{\mathcal{H}_{m}(\mathbb{C})} \mathbf{e}(-h x) \operatorname{det}(x+\sqrt{-1} g)^{-s} \operatorname{det}(x-\sqrt{-1} g)^{-s^{\prime}} d x .
$$

If $s=l>m$, we get the Fourier expansion of the holomorphic hermitian Eisenstein series $E_{2 l}^{(m)}(Z) \in$ $M_{2 l}\left(\Gamma_{K}^{(m)} ; \operatorname{det}^{-l}\right)$. If $H \in \Lambda_{m}(\mathcal{O})^{+}$, then

$$
\Xi(Y, H ; 2 l, 0)=\frac{(-1)^{m l} 2^{m(2 l-m+1)} \pi^{2 m l}}{\Gamma_{m}(2 l)}(\operatorname{det} H)^{2 l-m} \mathbf{e}(\sqrt{-1} H Y),
$$

where

$$
\Gamma_{m}(s)=\pi^{m(m-1) / 2} \prod_{i=1}^{m} \Gamma(s+1-i) .
$$

The $H$ th Fourier coefficient of $E_{2 l}^{(m)}(Z)$ for $H \in \Lambda_{m}(\mathcal{O})^{+}$is equal to

$$
\frac{(-1)^{m l} 2^{\left(4 m l-m^{2}+m\right) / 2} D^{-m(m-1) / 4} \pi^{2 m l}}{\Gamma_{m}(2 l) \prod_{i=1}^{m} L\left(2 l+1-i, \chi^{i-1}\right)}(\operatorname{det} H)^{(2 l-m)} \prod_{p \mid \gamma(H)} F_{p}\left(H ; p^{-2 l}\right) .
$$

It follows that the $H$ th Fourier coefficient of $E_{2 l}^{(m)}(Z)$ for $H \in \Lambda_{m}(\mathcal{O})^{+}$is equal to the product of

$$
A_{m} 2^{m} \prod_{i=1}^{m} L\left(i-2 l, \chi^{i-1}\right)^{-1}
$$

and

$$
|\gamma(H)|^{2 l-m} \prod_{p \mid \gamma(H)} F_{p}\left(H ; p^{-2 l}\right)=|\gamma(H)|^{l-(m / 2)} \prod_{p \mid \gamma(H)} \tilde{F}_{p}\left(H ; p^{l-(m / 2)}\right) .
$$

Here

$$
A_{m}= \begin{cases}1 & \text { if } m=2 n+1 \\ (-1)^{n} & \text { if } m=2 n\end{cases}
$$

Observe that

$$
\begin{aligned}
\prod_{p \mid \gamma(H)} \tilde{F}_{p}\left(H ; p^{l-(m / 2)}\right) & =\prod_{p \mid \gamma(H)} \underline{\chi}_{p}(\gamma(H))^{m-1} \tilde{F}_{p}\left(H ; p^{-l+(m / 2)}\right) \\
& =A_{m} \prod_{p \mid \gamma(H)} \tilde{F}_{p}\left(H ; p^{-l+(m / 2)}\right)
\end{aligned}
$$

by Lemma 2.2. We define the normalized Eisenstein series by

$$
\mathcal{E}_{2 l}^{(m)}(Z)=2^{-m} \prod_{i=1}^{m} L\left(i-2 l, \chi^{i-1}\right) \cdot E_{2 l}^{(m)}(Z) \in M_{2 l}\left(\Gamma_{K}^{(m)}, \operatorname{det}^{-l}\right) .
$$




\section{LIFTING OF HERMITIAN MODULAR FORMS}

When $m=2 n+1$, the $H$ th Fourier coefficient of $\mathcal{E}_{2 k^{\prime}+2 n}^{(2 n+1)}(Z)$ is equal to

$$
|\gamma(H)|^{k^{\prime}-(1 / 2)} \prod_{p \mid \gamma(H)} \tilde{F}_{p}\left(H ; p^{-k^{\prime}+(1 / 2)}\right)
$$

for any $H \in \Lambda_{2 n+1}(\mathcal{O})^{+}$and any sufficiently large integer $k^{\prime}$. When $m=2 n$, the $H$ th Fourier coefficient of $\mathcal{E}_{2 k^{\prime}+2 n}^{(2 n)}(Z)$ is equal to

$$
|\gamma(H)|^{k^{\prime}} \prod_{p \mid \gamma(H)} \tilde{F}_{p}\left(H ; p^{-k^{\prime}}\right)
$$

for any $H \in \Lambda_{2 n}(\mathcal{O})^{+}$and any sufficiently large integer $k^{\prime}$.

\section{Main theorems}

We first consider the case when $m=2 n$ is even. We refer to this case as Case E. In this case, let $f(\tau)=\sum_{N=1}^{\infty} a(N) q^{N} \in S_{2 k+1}\left(\Gamma_{0}(D), \chi\right)$ be a primitive form, whose $L$-function is given by

$$
L(f, s)=\prod_{p \nmid D}\left(1-a(p) p^{-s}+\chi(p) p^{2 k-2 s}\right)^{-1} \prod_{q \mid D}\left(1-a(q) q^{-s}\right)^{-1} .
$$

For each prime $p \nmid D$, we define the Satake parameter $\left\{\alpha_{p}, \beta_{p}\right\}=\left\{\alpha_{p}, \chi(p) \alpha_{p}^{-1}\right\}$ by

$$
\left(1-a(p) X+\chi(p) p^{2 k} X^{2}\right)=\left(1-p^{k} \alpha_{p} X\right)\left(1-p^{k} \beta_{p} X\right) .
$$

For $q \mid D$, we put $\alpha_{q}=q^{-k} a(q)$.

For each $H \in \Lambda_{2 n}(\mathcal{O})^{+}$, we put

$$
A(H)=|\gamma(H)|^{k} \prod_{p \mid \gamma(H)} \tilde{F}_{p}\left(H ; \alpha_{p}\right) .
$$

Here $p$ extends over all primes that divide $\gamma(H)$. We put

$$
F(Z)=\sum_{H \in \Lambda_{2 n}(\mathcal{O})^{+}} A(H) \mathbf{e}(H Z), \quad Z \in \mathcal{H}_{2 n}
$$

Then our main theorem for Case $\mathrm{E}$ is as follows.

Theorem 5.1 (Case E). Assume that $m=2 n$. Let $f(\tau), A(H)$ and $F(Z)$ be as above. Then we have $F \in S_{2 k+2 n}\left(\Gamma_{K}^{(2 n)}\right.$, $\left.\operatorname{det}^{-k-n}\right)$.

Now we consider the case when $m=2 n+1$ is odd. We refer to this case as Case O. In this case, let $f(\tau)=\sum_{N=1}^{\infty} a(N) q^{N} \in S_{2 k}\left(\mathrm{SL}_{2}(\mathbb{Z})\right)$ be a normalized Hecke eigenform, whose $L$-function is given by

$$
L(f, s)=\prod_{p}\left(1-a(p) p^{-s}+p^{2 k-1-2 s}\right)^{-1} .
$$

For each prime $p$, we define the Satake parameter $\left\{\alpha_{p}, \alpha_{p}^{-1}\right\}$ by

$$
\left(1-a(p) X+p^{2 k-1} X^{2}\right)=\left(1-p^{k-(1 / 2)} \alpha_{p} X\right)\left(1-p^{k-(1 / 2)} \alpha_{p}^{-1} X\right) .
$$

Put

$$
\begin{gathered}
A(H)=|\gamma(H)|^{k-(1 / 2)} \prod_{p \mid \gamma(H)} \tilde{F}_{p}\left(H ; \alpha_{p}\right), \quad H \in \Lambda_{2 n+1}(\mathcal{O})^{+}, \\
F(Z)=\sum_{H \in \Lambda_{2 n+1}(\mathcal{O})^{+}} A(H) \mathbf{e}(H Z), \quad Z \in \mathcal{H}_{2 n+1} .
\end{gathered}
$$




\section{T. IKEDA}

Then our main theorem for Case $\mathrm{O}$ is as follows.

Theorem 5.2 (Case O). Assume that $m=2 n+1$. Let $f(\tau), A(H)$ and $F(Z)$ be as above. Then we have $F \in S_{2 k+2 n}\left(\Gamma_{K}^{(2 n+1)}\right.$, $\left.\operatorname{det}^{-k-n}\right)$.

In both Case $\mathrm{E}$ and Case $\mathrm{O}$, the definition of $F(Z)$ is independent of the choice of $\alpha_{p}$ by Lemma 2.2. Observe that $F(Z)$ is absolutely convergent on $\mathcal{H}_{m}$ by Lemma 2.3 for any $m$. Since $F_{p}\left({ }^{\mathrm{t}} \bar{A} H A ; X\right)=F_{p}(H ; X)$ for any $H \in \Lambda_{m}(\mathcal{O})$ and any $A \in \mathrm{GL}_{m}(\mathcal{O})$, we have $\left.F\right|_{2 k+2 n} g=$ $(\operatorname{det} g)^{-k-n} F$ for any $g \in \Gamma_{K, \infty}^{(m)}$. We call $F(Z)$ the lift of $f(\tau)$ to $S_{2 k+2 n}\left(\Gamma_{K}^{(m)}\right.$, $\left.\operatorname{det}^{-k-n}\right)$ and denote it by $\operatorname{Lift}^{(m)}(f)$.

\section{Fourier-Jacobi expansions}

We define the Jacobi group $J_{m, r}(\mathcal{O})$ by

$$
\left\{M=\left(\begin{array}{ll|ll}
\mathbf{1}_{r} & * & * & * \\
0 & * & * & * \\
\hline 0 & 0 & \mathbf{1}_{r} & 0 \\
0 & * & * & *
\end{array}\right) \in \Gamma_{K}^{(m)} \mid \operatorname{det} M=1\right\} .
$$

We consider only the case $r=m-1$. Fix $S \in \Lambda_{m-1}(\mathcal{O})^{+}$.

For a holomorphic function $\phi\left(\tau, z_{1}, z_{2}\right)$ on $\mathfrak{H}_{1} \times \mathbb{C}^{m-1} \times \mathbb{C}^{m-1}$, we define a function on $\mathcal{H}_{m-1} \times$ $\mathfrak{H}_{1} \times \mathbb{C}^{m-1} \times \mathbb{C}^{m-1}$ by $\widetilde{\phi}(Z)=\mathbf{e}(S \omega) \phi\left(\tau, z_{1}, z_{2}\right)$. Here

$$
Z=\left(\begin{array}{cc}
\omega & z_{1} \\
{ }^{\mathrm{t}} z_{2} & \tau
\end{array}\right), \quad \omega \in \mathcal{H}_{m-1}, \tau \in \mathfrak{H}_{1}, z_{1}, z_{2} \in \mathbb{C}^{m-1} .
$$

We shall say that the function $\phi$ is a weak Jacobi form of index $S$ and weight $l$ if and only if

$$
\left.\widetilde{\phi}\right|_{l} M=\widetilde{\phi}
$$

for any $M \in J_{m, m-1}(\mathcal{O})$. A weak Jacobi form is called a Jacobi form if $\phi$ has a Fourier expansion

$$
\phi\left(\tau, z_{1}, z_{2}\right)=\sum_{x \in\left(\mathcal{O}^{\sharp}\right)^{m-1}} \sum_{N \in \mathbb{Z}} c(x, N) \mathbf{e}\left({ }^{\mathrm{t}} \bar{x} z_{1}+{ }^{\mathrm{t}} x z_{2}\right) \mathbf{e}(N \tau)
$$

such that $c(x, N)=0$ unless $N-{ }^{\mathrm{t}} \bar{x} S^{-1} x \geqslant 0$.

For each $\xi \in K^{m-1}$, we define the theta function $\theta_{[\xi]}\left(S ; \tau, z_{1}, z_{2}\right)$ by

$$
\theta_{[\xi]}\left(S ; \tau, z_{1}, z_{2}\right)=\sum_{x \in \mathcal{O}^{m-1}} \mathbf{e}\left({ }^{\mathrm{t}} \overline{(x+\xi)} S(x+\xi) \tau+{ }^{\mathrm{t}} \overline{(x+\xi) S} z_{1}+{ }^{\mathrm{t}}(x+\xi) S z_{2}\right) .
$$

Choose a complete representative $\Xi=\Xi(S)$ for $S^{-1}\left(\mathcal{O}^{\sharp}\right)^{m-1} / \mathcal{O}^{m-1}$.

Then a Jacobi form $\phi\left(\tau, z_{1}, z_{2}\right)$ of index $S$ can be expressed as the sum

$$
\begin{aligned}
\phi\left(\tau, z_{1}, z_{2}\right) & =\sum_{\xi \in \Xi} \theta_{[\xi]}\left(S ; \tau, z_{1}, z_{2}\right) \phi_{\xi}(\tau), \\
\phi_{\xi}(\tau) & =\sum_{\substack{N \in \mathbb{Z} \\
N-{ }^{\mathrm{t}} \bar{\xi} S \xi \geqslant 0}} c(S \xi, N) \mathbf{e}\left(\left(N-{ }^{\mathrm{t}} \bar{\xi} S \xi\right) \tau\right) .
\end{aligned}
$$

It is well known that, for each

$$
\gamma=\left(\begin{array}{ll}
a & b \\
c & d
\end{array}\right) \in \mathrm{SL}_{2}(\mathbb{Z})
$$




\section{LIFTING OF HERMITIAN MODULAR FORMS}

there exists a unitary representation $u_{S}: \mathrm{SL}_{2}(\mathbb{Z}) \rightarrow \mathrm{GL}\left(\mathbb{C}^{\Xi}\right)$ with kernel containing some congruence subgroup $\Gamma \subset \mathrm{SL}_{2}(\mathbb{Z})$ such that

$$
\theta_{[\xi]}\left(S ; \tau^{\prime}, z_{1}^{\prime}, z_{2}^{\prime}\right)=(c \tau+d)^{m-1} \sum_{\eta \in \Xi} \overline{u_{S}(\gamma)_{\eta \xi}} \mathbf{e}\left(S z_{1}(c \tau+d)^{-1} c^{\mathrm{t}} z_{2}\right) \theta_{[\eta]}\left(S ; \tau, z_{1}, z_{2}\right),
$$

where $\tau^{\prime}=(a \tau+b)(c \tau+d)^{-1}, z_{i}^{\prime}=z_{i}(c \tau+d)^{-1}, i=1,2$. It follows that

$$
\phi_{\xi}(\gamma\langle\tau\rangle)=(c \tau+d)^{l-m+1} \sum_{\eta \in \Xi} u_{S}(\gamma)_{\eta \xi} \phi_{\eta}(\tau)
$$

for any

$$
\gamma=\left(\begin{array}{ll}
a & b \\
c & d
\end{array}\right) \in \mathrm{SL}_{2}(\mathbb{Z})
$$

and any $\xi \in \Xi$.

Let $\mathfrak{F}(Z)$ be a holomorphic function on $\mathcal{H}_{m}$ that has a Fourier expansion

$$
\mathfrak{F}(Z)=\sum_{H \geqslant 0} \mathfrak{A}(H) \mathbf{e}(H Z) .
$$

Here $H$ extends over positive semi-definite elements of $\Lambda_{m}(\mathcal{O})$. We assume that $\mathfrak{A}\left({ }^{\mathrm{t}} \bar{X} H X\right)=\mathfrak{A}(H)$ for any $X \in \mathrm{GL}_{m}(\mathcal{O})$ and any $H \in \Lambda_{m}(\mathcal{O})$. For each $S \in \Lambda_{m-1}(\mathcal{O})$, we put

$$
\mathfrak{F}_{S}\left(\tau, z_{1}, z_{2}\right)=\sum_{H=\left(\begin{array}{cc}
S & x \\
\mathrm{t} \bar{x} & N
\end{array}\right)} \mathfrak{A}(H) \mathbf{e}(N \tau) \mathbf{e}\left({ }^{\mathrm{t}} \bar{x} z_{1}+{ }^{\mathrm{t}} x z_{2}\right) .
$$

Here $H$ extends over all positive semi-definite elements of $\Lambda_{m}(\mathcal{O})$ that are of the form

$$
H=\left(\begin{array}{cc}
S & x \\
{ }^{\mathrm{t}} \bar{x} & N
\end{array}\right) .
$$

As in [Ike01], we have an expansion

$$
\mathfrak{F}_{S}\left(\tau, z_{1}, z_{2}\right)=\sum_{\xi \in \Xi} \theta_{[\xi]}\left(S ; \tau, z_{1}, z_{2}\right) \mathfrak{F}_{S, \xi}(\tau)
$$

where

$$
\mathfrak{F}_{S, \xi}(\tau)=\sum_{\substack{N \in \mathbb{Z} \\
N-{ }^{\mathrm{t}} \bar{\xi} S \xi \geqslant 0}} \mathfrak{A}\left(\left(\begin{array}{cc}
S & S \xi \\
\mathrm{t} \frac{\bar{\xi}}{\xi} & N
\end{array}\right)\right) \mathbf{e}\left(\left(N-{ }^{\mathrm{t}} \bar{\xi} S \xi\right) \tau\right) .
$$

We call $\mathfrak{F}_{S, \xi}(\tau)$ the $(S, \xi)$-component of the Fourier-Jacobi expansion of $\mathfrak{F}(Z)$. Since the theta functions $\left\{\theta_{[\xi]}\left(S ; \tau, z_{1}, z_{2}\right) \mid \xi \in \Xi\right\}$ are linearly independent, $\mathfrak{F}_{S}\left(\tau, z_{1}, z_{2}\right)$ is a Jacobi form of index $S$ and weight $2 l$ if and only if

$$
\mathfrak{F}_{S, \xi}(\gamma\langle\tau\rangle)=(c \tau+d)^{l-m+1} \sum_{\eta \in \Xi} u_{S}(\gamma)_{\eta \xi} \mathfrak{F}_{S, \eta}(\tau)
$$

for any

$$
\gamma=\left(\begin{array}{ll}
a & b \\
c & d
\end{array}\right) \in \mathrm{SL}_{2}(\mathbb{Z})
$$

and any $\xi \in \Xi$.

\section{Vector-valued modular forms}

Let $\mathcal{K}_{p}=\mathrm{SL}_{2}\left(\mathbb{Z}_{p}\right)$ be the standard maximal compact subgroup of $\mathrm{SL}_{2}\left(\mathbb{Q}_{p}\right)$. We put $\mathcal{K}=\prod_{p} \mathcal{K}_{p}$. Let $(u, V)$ be a finite-dimensional continuous representation of $\mathcal{K}$. A $V$-valued modular form $\vec{h}(\tau)$ with 


\section{T. IKEDA}

type $u$ is a holomorphic function of $\tau \in \mathfrak{H}_{1}$ with values in $V$ that satisfies the following conditions (1) and (2):

(1) $\vec{h}(g\langle\tau\rangle)=(c \tau+d)^{\kappa} u(g)^{-1} \vec{h}(\tau)$ for any $g \in \mathrm{SL}_{2}(\mathbb{Z})$;

(2) $\vec{h}(\tau)$ has a Fourier expansion of the form

$$
\vec{h}(\tau)=\sum_{N=0}^{\infty} \vec{c}(N) q^{N / M}
$$

for some positive integer $M$.

We define vector-valued cusp forms similarly.

For each prime $p$, let $\mathcal{R}_{p}=\mathbb{C}\left[X_{p}, X_{p}^{-1}\right]$ be a copy of the Laurent polynomial ring. Put $\mathcal{R}=\bigotimes_{p} \mathcal{R}_{p}$. Then $\mathcal{R}$ is the ring of Laurent polynomials $\Phi(\mathbb{X})=\Phi\left(X_{2}, X_{3}, \ldots\right) \in \mathbb{C}\left[X_{2}, X_{2}^{-1}, X_{3}\right.$, $X_{3}^{-1}, \ldots$. Note that $\mathcal{R}$ is a unique factorization domain (UFD), although it is not noetherian. Let $a_{2}, a_{3}, \ldots, a_{p}, \ldots$ be non-zero complex numbers. Then the value of $\Phi(\mathbb{X})$ at $\left(X_{2}, X_{3}, \ldots, X_{p}, \ldots\right)=$ $\left(a_{2}, a_{3}, \ldots, a_{p}, \ldots\right)$ is denoted by $\Phi\left(\left\{a_{p}\right\}\right)$.

Lemma 7.1. Let $\Phi(\mathbb{X})$ be an element of $\mathcal{R}$. Assume that $\Phi\left(\left\{p^{-s}\right\}\right)=0$ for an infinite number of $s \in \mathbb{R}$. Then $\Phi(\mathbb{X})$ is identically 0 .

Proof. Write $\Phi(\mathbb{X})$ as a sum of monomials:

$$
\Phi(\mathbb{X})=\sum_{i=1}^{r} a_{i} \prod_{p} X_{p}^{e_{i, p}}
$$

Here $e_{i, p}=0$ for almost all $p$. Put $N_{i}=\prod_{p} p^{e_{i, p}}$. Then our assumption implies that $\sum a_{i} N_{i}^{-s}=0$ for infinitely many real numbers $s$. Since $N_{1}, \ldots, N_{r}$ are mutually distinct, we have $a_{1}=\cdots=a_{r}$ $=0$.

Definition 7.2. Let $h(\tau)$ be a modular form of weight $\kappa$ for some congruence subgroup $\Gamma$. Then we denote by $\mathcal{V}(h)$ the $\mathbb{C}$-vector space spanned by $\left\{h|\gamma| \gamma \in \mathrm{GL}_{2}(\mathbb{Q})^{+}\right\}$. If $u: \mathcal{K} \rightarrow \mathrm{GL}_{d}(\mathbb{C})$ is a representation of rank $d$, then $\mathcal{I}\left(\mathcal{V}(h)^{d}, u\right)$ is the space of $\mathbb{C}^{d}$-valued modular forms of type $u$ whose entries belong to $\mathcal{V}(h)$.

We first consider Case E. For Case E, we put

$$
E_{2 k+1, \chi}(\tau)=-\frac{B_{2 k+1, \chi}}{4 k+2}+\sum_{N=1}^{\infty}\left(\sum_{d \mid N} \chi(d) d^{2 k}\right) q^{N} \in M_{2 k+1}\left(\Gamma_{0}(D), \chi\right) .
$$

Note that $L\left(s, E_{2 k+1, \chi}\right)=\zeta(s) L(s-2 k, \chi)$. In particular, the Satake parameter of $E_{2 k+1, \chi}(\tau)$ is $\left\{p^{-k}, \chi(p) p^{k}\right\}$ for $p \nmid D$ and $\left\{q^{-k}\right\}$ for $q \mid D$.

Definition 7.3 (Case E). Let $k_{0}^{\prime}$ be some fixed large integer. We define a compatible family of Eisenstein series $\left\{\mathcal{F}_{2 k^{\prime}+1}(\tau)\right\}_{k^{\prime}} \geqslant k_{0}^{\prime}$ as follows. A compatible family of Eisenstein series is a family of modular forms

$$
\mathcal{F}_{2 k^{\prime}+1}(\tau)=b\left(2 k^{\prime}+1 ; 0\right)+\sum_{N \in \mathbb{Q}_{+}^{\times}} N^{k^{\prime}} b\left(2 k^{\prime}+1 ; N\right) q^{N}
$$

satisfying the following conditions (1), (2), and (3):

(1) $\mathcal{F}_{2 k^{\prime}+1} \in \mathcal{V}\left(E_{2 k^{\prime}+1, \chi}\right)$ for any integer $k^{\prime} \geqslant k_{0}^{\prime}$;

(2) for each $N \in \mathbb{Q}_{+}^{\times}$, there exists an element $\Phi_{N}(\mathbb{X}) \in \mathcal{R}$ such that

$$
b\left(2 k^{\prime}+1 ; N\right)=\Phi_{N}\left(\left\{p^{-k^{\prime}}\right\}\right) ;
$$

(3) there exists a congruence subgroup $\Gamma \subset \mathrm{SL}_{2}(\mathbb{Z})$ such that $\mathcal{F}_{2 k^{\prime}+1} \in M_{2 k^{\prime}+1}(\Gamma)$ for all $k^{\prime} \geqslant k_{0}^{\prime}$. 


\section{LIFTING OF HERMITIAN MODULAR FORMS}

As in [Ike01], we have to prove the following lemma.

LEMma 7.4. Let

$$
f(\tau)=\sum_{N>0} a(N) q^{N} \in S_{2 k+1}\left(\Gamma_{0}(D), \chi\right)
$$

be a primitive form and $\alpha_{p}$ a Satake parameter of $f(\tau)$. Assume that there are a finite-dimensional representation $\left(u, \mathbb{C}^{d}\right)$ of $\mathcal{K}$ and $\vec{\Phi}_{N}(\mathbb{X})={ }^{t}\left(\Phi_{1, N}(\mathbb{X}), \ldots, \Phi_{d, N}(\mathbb{X})\right) \in \mathcal{R}^{d}\left(N \in \mathbb{Q}_{+}^{\times}\right)$satisfying the following conditions (1) and (2).

(1) For each integer $k^{\prime} \geqslant k_{0}^{\prime}$, there exists a vector-valued modular form

$$
\overrightarrow{\mathcal{F}}_{2 k^{\prime}+1}(\tau)=\vec{b}\left(2 k^{\prime}+1 ; 0\right)+\sum_{N \in \mathbb{Q}_{+}^{\times}} N^{k^{\prime}} \vec{b}\left(2 k^{\prime}+1 ; N\right) q^{N}
$$

of type $u$.

(2) For each $i(1 \leqslant i \leqslant d)$, the $i$ th component $\mathcal{F}_{i, 2 k^{\prime}+1}(\tau)$ of $\overrightarrow{\mathcal{F}}_{2 k^{\prime}+1}(\tau)$ is a compatible family of Eisenstein series such that

$$
b_{i}\left(2 k^{\prime}+1 ; N\right)=\Phi_{i, N}\left(\left\{p^{-k^{\prime}}\right\}\right) .
$$

Here $\vec{b}\left(2 k^{\prime}+1 ; N\right)={ }^{\mathrm{t}}\left(b_{1}\left(2 k^{\prime}+1 ; N\right), \ldots, b_{d}\left(2 k^{\prime}+1 ; N\right)\right)$.

Put

$$
\vec{h}(\tau)=\sum_{N \in \mathbb{Q}_{+}^{\times}} N^{k} \vec{\Phi}_{N}\left(\left\{\alpha_{p}\right\}\right) q^{N} .
$$

Then we have $\vec{h}(\tau) \in \mathcal{I}\left(\mathcal{V}(f)^{d} ; u\right)$.

The lemma is proved at the end of $\S 9$, using results in the following two sections.

\section{Behavior of the Whittaker functions}

In this section, we will investigate the behavior of Whittaker functions on $\mathrm{GL}_{2}$, which will be used to prove Lemma 7.4 .

In this section, $F=F_{v}$ will denote a non-archimedean local field. We fix a non-trivial additive character $\psi$ of $F$. The maximal order of $F$, the prime ideal, and the order of the residue field are denoted by $\mathfrak{o}, \mathfrak{p}$, and $q$, respectively. We put $\mathcal{R}_{v}=\mathbb{C}\left[q^{s}, q^{-s}\right]$ and $\tilde{\mathcal{K}}_{v}=\mathrm{GL}_{2}(\mathfrak{o})$. The Borel subgroup of $\mathrm{GL}_{2}$ that consists of all upper triangular matrices is denoted by $\tilde{B}$. We put $B=\tilde{B} \cap \mathrm{SL}_{2}$ and $\mathcal{K}_{v}=\tilde{\mathcal{K}}_{v} \cap \mathrm{SL}_{2}$.

Let $\underline{\chi}, \underline{\chi}_{1}$ and $\underline{\chi}_{2}$ be characters of $F^{\times}$. The principal series representation $\tilde{I}\left(\underline{\chi}_{1} \otimes \underline{\chi}_{2}, s\right)=$ $\operatorname{Ind}_{\tilde{B}}^{\mathrm{GL}}\left(\underline{\chi}_{1}||^{s} \otimes \underline{\chi}_{2}||^{-s}\right)$ is the representation of $\mathrm{GL}_{2}$ that is induced from the character of $\tilde{B}$ given by

$$
\left(\begin{array}{cc}
a & b \\
0 & d
\end{array}\right) \mapsto \underline{\chi}_{1}(a) \underline{\chi}_{2}(d)\left|a d^{-1}\right|^{s}
$$

Similarly, the principal series representation $I(\underline{\chi}, s)=\operatorname{Ind}_{B}^{\mathrm{SL}_{2}}\left(\underline{\chi}||^{s}\right)$ is the representation of $\mathrm{SL}_{2}$ that is induced from the character of $B$ given by

$$
\left(\begin{array}{cc}
a & b \\
0 & a^{-1}
\end{array}\right) \mapsto \underline{\chi}(a)|a|^{s} .
$$

Then the restriction of $\tilde{I}\left(\underline{\chi}_{1} \otimes \underline{\chi}_{2}, s\right)$ to $\mathrm{SL}_{2}$ is canonically isomorphic to $I\left(\underline{\chi}_{1} \underline{\chi}_{2}^{-1}, 2 s\right)$.

In this section, we assume that $\underline{\chi}$ is a unitary character. 


\section{T. IKEDA}

An $\mathcal{R}_{v}$-valued function on $\mathrm{GL}_{2}$ can be regarded as a function $f(g, s)$ on $\mathrm{GL}_{2} \times \mathbb{C}$ such that $f(g, s) \in \mathcal{R}_{v}=\mathbb{C}\left[q^{s}, q^{-s}\right]$ for each $g \in \mathrm{GL}_{2}$. We say that a function on $\mathrm{GL}_{2} \times \mathbb{C}$ or on $\mathrm{SL}_{2} \times \mathbb{C}$ is right $\mathcal{K}_{v}$-finite if there is an open subgroup $\mathcal{K}_{v}^{c}$ of $\mathcal{K}_{v}$ such that $f(g, s)$ is right $\mathcal{K}_{v}^{c}$-invariant for any $s$. We also consider $\mathcal{R}_{v}$-valued functions and right $\mathcal{K}_{v}$-finiteness for $\mathrm{SL}_{2}$. Let $u$ be an irreducible representation of $\mathcal{K}_{v}$. Then we shall say that a right $\mathcal{K}_{v}$-finite function $f(g, s)$ has $\mathcal{K}_{v}$-type $u$ if $f(g, s)$ has $\mathcal{K}_{v}$-type $u$ for each $s \in \mathbb{C}$.

DeFinition 8.1. A right $\mathcal{K}_{v}$-finite $\mathcal{R}_{v}$-valued function $f(g, s)$ on $\mathrm{GL}_{2}$ is called a holomorphic section of $\tilde{I}\left(\underline{\chi}_{1} \underline{\nabla}_{\underline{\chi}_{2}}, s\right)$, if $f(g, s) \in \tilde{I}\left(\underline{\chi}_{1} \mathbb{\chi}_{2}, s\right)$ for each $s \in \mathbb{C}$. A holomorphic section $f(g, s)$ of $\tilde{I}\left(\underline{\chi}_{1} \underline{\chi}_{2}, s\right)$ is called a standard section if the restriction of $f(g, s)$ to $\mathcal{K}_{v} \times \mathbb{C}$ does not depend on $s \in \mathbb{C}$. We define holomorphic sections and standard sections of $I(\underline{\chi}, 2 s)$ similarly. We also define vector-valued holomorphic and standard sections of $I(\underline{\chi}, 2 s)$ in an obvious way.

For $f \in I(\underline{\chi}, 2 s)$ or $f \in \tilde{I}\left(\underline{\chi}_{1} \otimes \underline{\chi}_{2}, s\right)$, we put

$$
\mathrm{Wh}_{\psi}(f)(g)=\int_{F} f\left(w\left(\begin{array}{ll}
1 & x \\
0 & 1
\end{array}\right) g\right) \overline{\psi(x)} d x,
$$

where

$$
w=\left(\begin{array}{rr}
0 & -1 \\
1 & 0
\end{array}\right)
$$

The Haar measure $d x$ is the self-dual measure with respect to the additive character $\psi$. This integral is absolutely convergent for $\operatorname{Re}(s)>0$. If $f(g, s)$ is a holomorphic section of $I(\underline{\chi}, 2 s)$, then $\mathrm{Wh}_{\psi}(f(g, s))$ is an $\mathcal{R}_{v}$-valued function on $\mathrm{SL}_{2}$. Thus $\mathrm{Wh}_{\psi}(f)$ is meaningful for any $s \in \mathbb{C}$. We denote $\mathcal{W}_{\psi}(I(\underline{\chi}, 2 s))=\left\{\mathrm{Wh}_{\psi}(f) \mid f \in I(\underline{\chi}, 2 s)\right\}$ for each $s \in \mathbb{C}$. It is known that $\mathcal{W}_{\psi}(I(\underline{\chi}, 2 s)) \neq(0)$ for any $s \in \mathbb{C}$. If $I(\underline{\chi}, 2 s)$ is irreducible, then $\mathcal{W}_{\psi}(I(\underline{\chi}, 2 s))$ is equal to the Whittaker space of $I(\underline{\chi}, 2 s)$.

We need to investigate the behavior of Whittaker functions at the points of reducibility of $I(\underline{\chi}, 2 s)$. Since $I\left(\underline{\chi}||^{2 s^{\prime}}, 2 s\right) \simeq I\left(\underline{\chi}, 2\left(s+s^{\prime}\right)\right)$, we have only to consider real $s$ by changing $\underline{\chi}$ if necessary. If $\underline{\chi}^{2} \neq 1$, then there are no real points of reducibility of $I(\underline{\chi}, 2 s)$. When $\underline{\chi}=1$, the real points of reducibility of $I(\mathbf{1}, 2 s)$ are $s= \pm 1 / 2$. Then $I(\mathbf{1}, 1)$ contains the Steinberg representation St, and the quotient of $I(\mathbf{1}, 1) /$ St is the trivial representation $\mathbf{1}$; and $I(\mathbf{1},-1)$ contains $\mathbf{1}$, and $I(\mathbf{1},-1) / \mathbf{1} \simeq$ St. We have

$$
\begin{aligned}
\operatorname{Ker}\left(\mathrm{Wh}_{\psi}: I(\mathbf{1}, 1) \rightarrow \mathcal{W}_{\psi}(I(\mathbf{1}, 1))\right) & =\{0\}, \\
\operatorname{Ker}\left(\mathrm{Wh}_{\psi}: I(\mathbf{1},-1) \rightarrow \mathcal{W}_{\psi}(I(\mathbf{1},-1))\right) & =\mathbf{1} .
\end{aligned}
$$

If $\underline{\chi}^{2}=1, \underline{\chi} \neq 1$, then the real point of reducibility of $I(\underline{\chi}, 2 s)$ is $s=0$. In this case, $I(\underline{\chi}, 0)$ is the direct sum of two irreducible representations $I(\underline{\chi}, 0)^{+} \oplus \bar{I}(\underline{\chi}, 0)^{-}$. This decomposition is described in terms of the normalized intertwining operator $M^{*}(2 s, \underline{\bar{\chi}}, \psi)=\varepsilon(2 s, \underline{\chi}, \psi) M(2 s, \underline{\chi})$. Note that $M^{*}\left(2 s, \underline{\chi}, \psi_{a}\right)=\underline{\chi}(a)|a|^{2 s} M^{*}(2 s, \underline{\chi}, \psi)$, where $\psi_{a}(x)=\psi(a x)$. The irreducible constituents $I(\underline{\chi}, 0)^{+}$ and $I(\underline{\chi}, 0)^{-}$are the spaces of elements of $I(\underline{\chi}, 0)$ on which $M^{*}(0, \underline{\chi}, \psi)$ acts by 1 and by -1 , respectively. Furthermore,

$$
\operatorname{Ker}\left(\mathrm{Wh}_{\psi}: I(\underline{\chi}, 0) \rightarrow \mathcal{W}_{\psi}(I(\underline{\chi}, 0))\right)=I(\underline{\chi}, 0)^{-} .
$$

Lemma 8.2. Let $\underline{\chi}$ be a character of $F^{\times}$such that $\underline{\chi}^{2}=1$. Any irreducible representation of $\mathcal{K}_{v}$ is multiplicity-free in $\tilde{I}\left(1 \otimes \underline{\chi}^{-1}, s\right)$. Moreover, the set of irreducible representations of $\mathcal{K}_{v}$ that occur in $\tilde{I}\left(\mathbf{1} \otimes \underline{\chi}^{-1}, s\right)$ is independent of $s$.

Proof. It is enough to consider the restriction of $\tilde{I}\left(\mathbf{1} \otimes \underline{\chi}^{-1}, s\right)$ to $\mathrm{SL}_{2}$. Note that the restriction is isomorphic to $I(\underline{\chi}, 2 s)$. Put $\mathcal{B}=B \cap \mathcal{K}_{v}$ and

$$
\mathcal{N}=\left\{\left(\begin{array}{ll}
1 & n \\
0 & 1
\end{array}\right) \mid n \in \mathfrak{o}\right\} .
$$




\section{LIFTING OF HERMITIAN MODULAR FORMS}

We are going to prove that the induced representation $\operatorname{Ind}_{\mathcal{B}}^{\mathcal{K}_{v}} \underline{\chi}$ is multiplicity-free. Let $\mathcal{H}\left(\mathcal{B} \backslash \mathcal{K}_{v} / \mathcal{B} ; \underline{\chi}\right)$ be the Hecke algebra that consists of all locally constant functions $\phi$ on $\mathcal{K}_{v}$ such that $\phi\left(b_{1} k b_{2}\right)=$ $\underline{\chi}\left(b_{1} b_{2}\right) \phi(k)$ for any $b_{1}, b_{2} \in \mathcal{B}, k \in \mathcal{K}_{v}$. It is enough to prove that $\mathcal{H}\left(\mathcal{B} \backslash \mathcal{K}_{v} / \mathcal{B} ; \underline{\chi}\right)$ is commutative.

We consider the anti-involution

$$
\tau\left(\begin{array}{cc}
x & y \\
z & w
\end{array}\right)=\left(\begin{array}{ll}
w & y \\
z & x
\end{array}\right)
$$

of $\mathcal{K}_{v}$. We shall prove that, for any

$$
k=\left(\begin{array}{cc}
x & y \\
z & w
\end{array}\right) \in \mathcal{K}_{v},
$$

there exist $b_{1}, b_{2} \in \mathcal{B}$ such that ${ }^{\tau} k=b_{1} k b_{2}$ and $\underline{\chi}\left(b_{1} b_{2}\right)=1$. If $z \in \mathfrak{o}^{\times}$, then one can easily find $n_{1}, n_{2} \in \mathcal{N}$ such that ${ }^{\tau} k=n_{1} k n_{2}$. If $z \in \mathfrak{p}$, then

$$
k=b\left(\begin{array}{cc}
1 & 0 \\
z^{\prime} & 1
\end{array}\right)
$$

for some $z^{\prime} \in \mathfrak{p}$ and $b \in \mathcal{B}$. In this case one can choose $b_{1}=b^{-1}, b_{2}={ }^{\tau} b$. This completes the proof of the first assertion. The last assertion of the lemma is clear.

Let $\left(u, \mathbb{C}^{d}\right)$ be an irreducible representation of $\mathcal{K}_{v}$, which occurs in $I(\underline{\chi}, 2 s)$. There is a vectorvalued standard section

$$
f_{u}(g, s)=\left(f_{u, 1}(g, s), \ldots, f_{u, d}(g, s)\right)
$$

of $I(\underline{\chi}, 2 s)$ such that $f_{u}(g k, s)=f_{u}(g, s) u(k)$ for any $k \in \mathcal{K}_{v}$. By Lemma 8.2, such a section is unique up to a scalar multiplication. The standard section $f_{u}(g, s)$ can be uniquely extended to a standard section $\tilde{f}_{u}(g, s)$ of $\tilde{I}(\mathbf{1} \otimes \underline{\chi}, s)$. Set

$$
\begin{aligned}
& W_{u}(g, s)=\mathrm{Wh}_{\psi}\left(f_{u}(g, s)\right)=\left(\mathrm{Wh}_{\psi}\left(f_{u, 1}(g, s)\right), \ldots, \mathrm{Wh}_{\psi}\left(f_{u, d}(g, s)\right)\right), \\
& \tilde{W}_{u}(g, s)=\mathrm{Wh}_{\psi}\left(\tilde{f}_{u}(g, s)\right)=\left(\mathrm{Wh}_{\psi}\left(\tilde{f}_{u, 1}(g, s)\right), \ldots, \mathrm{Wh}_{\psi}\left(\tilde{f}_{u, d}(g, s)\right)\right) .
\end{aligned}
$$

Let $\alpha_{\psi, u}(s) \in \mathcal{R}_{v}$ be the generators of the ideal of $\mathcal{R}_{v}$ generated by $\left\{W_{u, i}(g, s) \mid g \in \mathrm{SL}_{2}, 1 \leqslant i \leqslant d\right\}$. Set $W_{u}^{\prime}(g, s)=\alpha_{\psi, u}(s)^{-1} W_{u}(g, s)$. Then we have $W_{u}^{\prime}\left(g k, s_{0}\right)=W_{u}^{\prime}\left(g, s_{0}\right) u(k)$ for any $k \in \mathcal{K}_{v}$ and $s_{0} \in \mathbb{C}$. In particular, $\left\{W_{u, i}^{\prime}(g, s) \mid 1 \leqslant i \leqslant d\right\}$ is linearly independent. It follows that $f_{u, i}\left(g, s_{0}\right) \in$ $\operatorname{Ker}\left(\mathrm{Wh}_{\psi}\right)$ if and only if $\alpha_{\psi, u}\left(s_{0}\right)=0$ for any $1 \leqslant i \leqslant d$. Note that $I\left(\underline{\chi}, 2 s_{0}\right)$ is reducible in this case, since $\operatorname{Ker}\left(\mathrm{Wh}_{\psi}\right) \neq I\left(\underline{\chi}, 2 s_{0}\right)$.

Similarly, let $\tilde{\alpha}_{u}(s) \in \mathcal{R}_{v}$ be the generators of the ideal of $\mathcal{R}_{v}$ generated by $\left\{\tilde{W}_{u, i}(g, s) \mid g \in\right.$ $\left.\mathrm{GL}_{2}, i=1, \ldots, d\right\}$. Then, $\tilde{\alpha}_{u}\left(s_{0}\right)=0$ if and only if $u=1$ and $q^{2 s_{0}}=q^{-1}$. Note that $\tilde{\alpha}_{u}(s)$ does not depend on the choice of $\psi$.

Lemma 8.3. If $u=\mathbf{1}$ and $\psi$ is of order 0 , then $\tilde{\alpha}_{u}(s)=\alpha_{\psi, u}(s)$. For each irreducible representation $u$ of $\mathcal{K}_{v}$, there is an element $a \in k^{\times}$such that $\tilde{\alpha}_{u}(s)=\alpha_{\psi_{a}, u}(s)$. Here, $\psi_{a}(x)=\psi(a x)$.

Proof. The first part can be checked by direct calculation. For the proof of the latter part, we may assume that $u \neq 1$. We may also assume that $\underline{\chi}$ is either the trivial character or a ramified character of order 2. If $\underline{\chi}=\mathbf{1}$ and $\alpha_{\psi, u}(s) \neq \tilde{\alpha}_{u}(s)$, then the $\mathcal{K}_{v^{-}}$type $u$ occur in $I\left(\underline{\chi}^{\mathrm{ur}}, 0\right)^{-}$, where $\underline{\chi}^{\mathrm{ur}}$ is the unramified character of order 2 . Note that $I\left(\underline{\chi}^{\mathrm{ur}}, 0\right)=I(\mathbf{1}, \pi \sqrt{-1} / \log q)$. In this case, one can choose $a \in k^{\times}$such that $\underline{\chi}^{\mathrm{ur}}(a)=-1$.

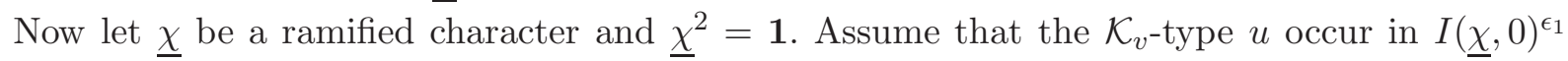
and $I\left(\underline{\chi \chi}^{\mathrm{ur}}, \overline{0}\right)^{\epsilon_{2}}$, where $\epsilon_{1}, \epsilon_{2} \in\{ \pm 1\}$. Choose $a \in k^{\times}$such that $\underline{\chi}(a)=\epsilon_{1}$ and $\underline{\chi \chi}^{\mathrm{ur}}(a)=\epsilon_{2}$. Then $\psi_{a}$ satisfies the condition of the lemma. 


\section{T. IKEDA}

Definition 8.4. A right $\mathcal{K}_{v}$-finite $\mathcal{R}_{v}$-valued function $W(g, s)$ on $\mathrm{SL}_{2}$ is an $\mathcal{R}_{v}$-valued Whittaker function if $W\left(g, s_{0}\right) \in \mathcal{W}_{\psi}\left(I\left(\underline{\chi}, 2 s_{0}\right)\right)$ unless $I\left(\underline{\chi}, 2 s_{0}\right)$ is reducible. Similarly, a right $\mathcal{K}_{v^{-}}$finite $\mathcal{R}_{v^{-}}$ valued function $\tilde{W}(g, s)$ on $\mathrm{GL}_{2}$ is an $\mathcal{R}_{v}$-valued Whittaker function if $\tilde{W}\left(g, s_{0}\right) \in \mathcal{W}_{\psi}\left(\tilde{I}\left(\mathbf{1} \otimes \underline{\chi}, s_{0}\right)\right)$ unless $\tilde{I}\left(\mathbf{1} \otimes \underline{\chi}, s_{0}\right)$ is reducible. When we need to refer to $\psi$, we shall say ' $\mathcal{R}_{v}$-valued $\psi$-Whittaker function'.

Lemma 8.5. Let $u$ be an irreducible representation of $\mathcal{K}_{v}$. Set

$$
W_{u, i}^{\prime}(g, s)=\alpha_{\psi, u}(s)^{-1} W_{u, i}(g, s), \quad \tilde{W}_{u, i}^{\prime}(g, s)=\tilde{\alpha}_{u}(s)^{-1} \tilde{W}_{u, i}(g, s) .
$$

Then the $\mathcal{R}_{v}$-module generated by the $\mathcal{R}_{v}$-valued Whittaker function of $I(\underline{\chi}, 2 s)$ with $\mathcal{K}_{v}$-type $u$ is generated by $\left\{W_{u, i}^{\prime}(g, s) \mid 1 \leqslant i \leqslant d\right\}$. Similarly, the $\mathcal{R}_{v}$-module generated by the $\mathcal{R}_{v^{-v a l u e d}}$ Whittaker function of $\tilde{I}(\mathbf{1} \otimes \underline{\chi}, s)$ with $\mathcal{K}_{v}$-type $u$ is generated by $\left\{\tilde{W}_{u, i}^{\prime}(g, s) \mid 1 \leqslant i \leqslant d\right\}$.

Proof. We prove only the first part. Note that $\left\{W_{u, i}^{\prime}\left(g, s_{0}\right) \mid 1 \leqslant i \leqslant d\right\}$ is linearly independent for any $s_{0} \in \mathbb{C}$. Let $W(g, s)$ be an $\mathcal{R}_{v}$-valued Whittaker function of $I(\underline{\chi}, 2 s)$ with $\mathcal{K}_{v}$-type $u$. Then there exists $c_{i}(s) \in \mathcal{Q}\left(\mathcal{R}_{v}\right)$ such that $W(g, s)=\sum_{i=1}^{d} c_{i}(s) W_{u, i}^{\prime}(g, s)$, where $\mathcal{Q}(\mathcal{R})$ is the quotient field of $\mathcal{R}_{v}$. Assume that $c_{i}(s) \notin \mathcal{R}_{v}$. Then there exists $s_{0} \in \mathbb{C}$ such that $\operatorname{ord}_{s=s_{0}} c_{i}(s)>0$, where ord is the order of the pole. We may assume that $e=\operatorname{ord}_{s=s_{0}} c_{1}(s) \geqslant \operatorname{ord}_{s=s_{0}} c_{i}(s)$ for $i=2, \ldots, d$. Then

$$
\sum_{i=1}^{d}\left(\left(s-s_{0}\right)^{e} c_{i}(s)\right)_{s=s_{0}} \cdot W^{\prime}\left(g, s_{0}\right)=0
$$

which contradicts the linear independence of $\left\{W_{u, i}^{\prime}\left(g, s_{0}\right) \mid 1 \leqslant i \leqslant d\right\}$.

Let $W(g, s)$ be an $\mathcal{R}_{v}$-valued $\psi$-Whittaker function of $I(\underline{\chi}, 2 s)$ with $\mathcal{K}_{v}$-type $u$. If $s$ is not a point of reducibility of $I(\underline{\chi}, 2 s)$, one can extend $W(g, s)$ to a $\psi$-Whittaker function $\tilde{W}(g, s)$ of $\tilde{I}(\mathbf{1} \otimes \underline{\chi}, s)$.

Lemma 8.6. Set $\beta_{\psi, u}(s)=\tilde{\alpha}_{u}(s)^{-1} \alpha_{\psi, u}(s)$. Then $\beta_{\psi, u}(s) \tilde{W}(g, s)$ is an $\mathcal{R}_{v^{-v a l u e d}}$ Whittaker function of $\tilde{I}(1 \otimes \underline{\chi}, s)$.

Proof. This lemma follows immediately from the fact that the $\mathcal{R}_{v^{-}}$module generated by the $\mathcal{R}_{v^{-}}$ valued Whittaker function of $I(\underline{\chi}, 2 s)$ with $\mathcal{K}_{v}$-type $u$ is generated by $\left\{\alpha_{\psi, u}(s)^{-1} W_{u, i}(g, s) \mid i=\right.$ $1, \ldots, d\}$.

Lemma 8.7. Let $\mathfrak{N}_{0} \subset \mathbb{R}$ be an infinite subset such that $I(\underline{\chi}, 2 s)$ is irreducible for any $s \in \mathfrak{N}_{0}$.

(1) Let $W(g, s)$ be a right $\mathcal{K}_{v}$-finite $\mathcal{R}_{v}$-valued function on $\mathrm{SL}_{2}$ such that $W(g, s) \in \mathcal{W}_{\psi}(I(\underline{\chi}, 2 s))$ for any $s \in \mathfrak{N}_{0}$. Then $W(g, s)$ is an $\mathcal{R}_{v}$-valued Whittaker function of $I(\underline{\chi}, 2 s)$.

(2) Let $\tilde{W}(g, s)$ be a right $\tilde{\mathcal{K}}_{v}$-finite $\mathcal{R}_{v}$-valued function on $\mathrm{GL}_{2}$ such that $\tilde{W}(g, s) \in \mathcal{W}_{\psi}(\tilde{I}(\mathbf{1} \otimes \underline{\chi}, s))$ for any $s \in \mathfrak{N}_{0}$. Then $\tilde{W}(g, s)$ is an $\mathcal{R}_{v}$-valued Whittaker function of $\tilde{I}(\mathbf{1} \otimes \underline{\chi}, s)$.

Proof. We will prove only part (1), as the proof of part (2) is similar. Let $\left(u, \mathbb{C}^{d}\right)$ be an irreducible representation of $\mathcal{K}_{v}$. By standard arguments, it is enough to prove the following.

$\left(1^{\prime}\right)$ Let $W(g, s)=\left(W_{1}(g, s), \ldots, W_{d}(g, s)\right)$ be a vector of $\mathcal{R}_{v}$-valued functions on $\mathrm{SL}_{2}$ such that $W_{i}\left(g, s_{0}\right) \in \mathcal{W}_{\psi}\left(I\left(\underline{\chi}, 2 s_{0}\right)\right)(1 \leqslant i \leqslant d)$ for any $s_{0} \in \mathfrak{N}_{0}$ and $W(g k, s)=W(g, s) u(k)$ for any $k \in \mathcal{K}_{v}$. Then $\left.W_{i} \overline{(g}, s\right) \in \mathcal{W}_{\psi}\left(I\left(\underline{\chi}, 2 s_{0}\right)\right)(1 \leqslant i \leqslant d)$ for any $s_{0} \in \mathbb{C}$ such that $I\left(\underline{\chi}, 2 s_{0}\right)$ is irreducible.

By Lemma 8.2, there exists an element $\varphi(s) \in \mathcal{R}_{v}$ such that $W(g, s)=\varphi(s) \alpha_{\psi, u}(s)^{-1} W_{u}(g, s)$. Since the zero of $\alpha_{\psi, u}(s)$ is a point of reducibility of $I(\underline{\chi}, 2 s)$, the lemma follows. 


\section{LIFTING OF HERMITIAN MODULAR FORMS}

\section{Adelic compatible family}

We put $\hat{\mathbb{Z}}=\prod_{p} \mathbb{Z}_{p}$ and $\hat{\mathbb{Z}}^{\times}=\prod_{p} \mathbb{Z}_{p}^{\times}$. Let

$$
\tilde{B}=\left\{\left(\begin{array}{ll}
* & * \\
0 & *
\end{array}\right) \in \mathrm{GL}_{2}\right\}
$$

be the standard Borel subgroup of $\mathrm{GL}_{2}$. Put $\mathcal{K}=\mathrm{SL}_{2}(\hat{\mathbb{Z}}), \tilde{\mathcal{K}}=\mathrm{GL}_{2}(\hat{\mathbb{Z}})$, and

$$
\tilde{\mathcal{K}}_{0}=\left\{\left(\begin{array}{ll}
a & b \\
c & d
\end{array}\right) \in \mathrm{GL}_{2}(\hat{\mathbb{Z}}) \mid c \in D \mathbb{Z}\right\} .
$$

Recall that, given $h \in M_{2 k+1}\left(\Gamma_{0}(D), \chi\right)$, one can define an adelic cusp form $h^{\sharp}$ on $\mathrm{GL}_{2}(\mathbb{A})$ by the formula

$$
h^{\sharp}(g)=\underline{\chi}(d)\left(f \mid g_{\infty}\right)(\sqrt{-1})
$$

for $g=\gamma g_{\infty} g_{0} \in \mathrm{GL}_{2}(\mathbb{A})$ with $\gamma \in \mathrm{GL}_{2}(\mathbb{Q}), g_{\infty} \in \mathrm{GL}_{2}^{+}(\mathbb{R})$, and

$$
g_{0}=\left(\begin{array}{ll}
a & b \\
c & d
\end{array}\right) \in \tilde{\mathcal{K}}_{0}
$$

For a primitive form $f \in S_{2 k+1}\left(\Gamma_{0}(D), \chi\right)$, we put $\mathbf{f}=f^{\sharp}$. We also put $\mathbf{E}_{2 k^{\prime}+1, \chi}=\left(E_{2 k^{\prime}+1, \chi}\right)^{\sharp}$. Let $\pi \simeq \bigotimes_{v}^{\prime} \pi_{v}$ be an irreducible cuspidal automorphic representation of $G_{2}(\mathbb{A})$ generated by $\mathbf{f}$. Then the central character of $\pi$ is $\underline{\chi}$, and $\pi_{\infty}$ is the (limit of the) discrete series representation of $\mathrm{GL}_{2}(\mathbb{R})$ with minimal weight $\pm(2 k+1)$. The $p$-component $\pi_{p}$ is isomorphic to

$$
\tilde{I}\left(1 \otimes \underline{\chi}_{p}, s_{0, p}\right)=\operatorname{Ind}_{\tilde{B}\left(\mathbb{Q}_{p}\right)}^{\mathrm{GL}_{2}\left(\mathbb{Q}_{p}\right)}\left(|\cdot|^{s_{0, p}} \otimes \underline{\chi}_{p}|\cdot|^{-s_{0, p}}\right),
$$

where $s_{0, p} \in \mathbb{C}$ is a complex number such that $e^{-s_{0, p} \cdot \log p}=\alpha_{p}$. Note that $\operatorname{Re}\left(s_{0, p}\right)=0$ by the Ramanujan conjecture.

Let $\mathcal{V}(\mathbf{f})$ be the $\mathbb{C}$-vector space spanned by the right translates of $\mathbf{f}$ by $\mathrm{GL}_{2}\left(\mathbb{A}_{\mathrm{f}}\right)$. Then $\mathcal{V}(\mathbf{f})$ can be regarded as the representation space of $\bigotimes_{p<\infty}^{\prime} \pi_{p}$. We define $\mathcal{V}\left(\mathbf{E}_{2 k^{\prime}+1, \chi}\right)$ similarly; $\mathcal{V}\left(\mathbf{E}_{2 k^{\prime}+1, \chi}\right)$ is isomorphic to $\bigotimes_{p<\infty}^{\prime} \tilde{I}\left(\mathbf{1} \otimes \underline{\chi}_{p}, k^{\prime}\right)$.

For each $\mathbf{h} \in \mathcal{V}(\mathbf{f})$ or $\mathbf{h} \in \mathcal{V}\left(\mathbf{E}_{2 k^{\prime}+1, \chi}\right)$, we can associate a function $\xi(\mathbf{h})$ on $\mathfrak{H}$ by

$$
\xi(\mathbf{h})(\tau)=\mathbf{h}\left(g_{\infty}\right) j\left(g_{\infty}, \sqrt{-1}\right)^{2 k+1}
$$

for $g_{\infty} \in \mathrm{GL}_{2}(\mathbb{R})^{+} \subset \mathrm{GL}_{2}(\mathbb{A}), g_{\infty}(\sqrt{-1})=\tau$.

Lemma 9.1. The map $\xi$ gives surjections $\mathcal{V}\left(\mathbf{E}_{2 k^{\prime}+1, \chi}\right) \rightarrow \mathcal{V}\left(E_{2 k^{\prime}+1, \chi}\right)$ and $\mathcal{V}(\mathbf{f}) \rightarrow \mathcal{V}(f)$.

Proof. This follows from the equality $\mathrm{GL}_{2}\left(\mathbb{A}_{\mathrm{f}}\right)=\mathrm{GL}_{2}(\mathbb{Q})^{+} \cdot \tilde{\mathcal{K}}_{0}$, which follows from the strong approximation of $\mathrm{SL}_{2}$.

One can define an action $\rho$ of $\mathrm{SL}_{2}\left(\mathbb{A}_{\mathrm{f}}\right)$ on $\mathcal{V}(f)$ or on $\mathcal{V}\left(E_{2 k^{\prime}+1, \chi}\right)$ by $\rho(g) h=h \mid \gamma^{-1}$, for $\gamma \in$ $\mathrm{SL}_{2}(\mathbb{Q})$ sufficiently close to $g \in \mathrm{SL}_{2}\left(\mathbb{A}_{\mathrm{f}}\right)$. Note that $\mathrm{SL}_{2}(\mathbb{Q})$ is dense in $\mathrm{SL}_{2}\left(\mathbb{A}_{\mathrm{f}}\right)$ by the strong approximation theorem.

The maps $\xi: \mathcal{V}\left(\mathbf{E}_{2 k^{\prime}+1, \chi}\right) \rightarrow \mathcal{V}\left(E_{2 k^{\prime}+1, \chi}\right)$ and $\xi: \mathcal{V}(\mathbf{f}) \rightarrow \mathcal{V}(f)$ are $\mathrm{SL}_{2}\left(\mathbb{A}_{\mathrm{f}}\right)$ equivariant. In particular, $\xi: \mathcal{V}\left(\mathbf{E}_{2 k^{\prime}+1, \chi}\right) \rightarrow \mathcal{V}\left(E_{2 k^{\prime}+1, \chi}\right)$ is an isomorphism for sufficiently large $k^{\prime}$, since the restriction of $\tilde{I}\left(\mathbf{1} \otimes \underline{\chi}_{p}, s\right)$ to $\mathrm{SL}_{2}\left(\mathbb{Q}_{p}\right)$ is irreducible for $\operatorname{Re}(s)>1 / 2$.

For $x=\left(x_{v}\right)_{v} \in \mathbb{A}$, we set $\mathbf{e}_{\mathbb{A}}(x)=\prod_{p} \mathbf{e}_{p}\left(x_{p}\right) \cdot \mathbf{e}\left(x_{\infty}\right)$. Then for any non-trivial additive character $\psi$ of $\mathbb{A} / \mathbb{Q}$, there is a rational number $t \in \mathbb{Q}^{\times}$such that $\psi(x)=\mathbf{e}_{\mathbb{A}}(t x)$. We assume that $t>0$. For $\mathbf{h} \in \mathcal{V}\left(\mathbf{E}_{2 k^{\prime}+1, \chi}\right)$ or $\mathbf{h} \in \mathcal{V}(\mathbf{f})$, the Whittaker function $W_{\psi, \mathbf{h}}$ of $\mathbf{h}$ is defined by

$$
W_{\psi, \mathbf{h}}(g)=\int_{\mathbb{Q} \backslash \mathbb{A}} \mathbf{h}\left(\left(\begin{array}{ll}
1 & x \\
0 & 1
\end{array}\right) g\right) \overline{\psi(x)} d x .
$$




\section{T. IKEDA}

If the weight of $h$ is $2 k+1$, then the Whittaker function $W_{\psi, \mathbf{h}}$ can be decomposed into a finite part and an infinite part:

$$
W_{\psi, \mathbf{h}}=W_{\psi, \mathbf{f}}^{0} W_{\psi, 2 k+1}^{\infty} .
$$

Here, the infinite part $W_{\psi, 2 k+1}^{\infty}$ is given by

$$
\begin{aligned}
& W_{\psi, 2 k+1}^{\infty}\left(\left(\begin{array}{ll}
a & x \\
0 & 1
\end{array}\right)\left(\begin{array}{cc}
\cos \theta & -\sin \theta \\
\sin \theta & \cos \theta
\end{array}\right)\right) \\
& = \begin{cases}e^{2 \pi \sqrt{-1} t x}(t a)^{k+(1 / 2)} e^{-2 \pi t a} e^{\sqrt{-1}(2 k+1) \theta} & \text { if } a>0, \\
0 & \text { if } a<0 .\end{cases}
\end{aligned}
$$

If $\psi^{\prime}(x)=\psi(a x)$ for $a \in \mathbb{Q}_{+}^{\times}$, then we have

$$
W_{\psi^{\prime}, \mathbf{h}}^{0}(g)=W_{\psi, \mathbf{h}}^{0}\left(\left(\begin{array}{cc}
a & 0 \\
0 & 1
\end{array}\right) g\right), \quad g \in \mathrm{GL}_{2}\left(\mathbb{A}_{\mathrm{f}}\right) .
$$

Definition 9.2. Let $k_{0}^{\prime}$ be some fixed large integer. An adelic compatible family of Eisenstein series is a family $\left\{\mathbf{F}_{2 k^{\prime}+1}\right\}_{k^{\prime}} \geqslant k_{0}^{\prime}$ that satisfies the following conditions (1) and (2):

(1) $\mathbf{F}_{2 k^{\prime}+1} \in \mathcal{V}\left(\mathbf{E}_{2 k^{\prime}+1, \chi}\right)$ for each $k^{\prime} \geqslant k_{0}^{\prime}$;

(2) there is an $\mathcal{R}$-valued function $\mathbb{W}_{\psi}(g ; \mathbb{X})$ on $\mathrm{GL}_{2}\left(\mathbb{A}_{\mathrm{f}}\right)$ such that $W_{\psi, \mathbf{F}_{2 k^{\prime}+1}}(g)=\mathbb{W}_{\psi}\left(g ;\left\{p^{-k^{\prime}}\right\}\right)$ for each $k^{\prime}$.

This definition does not depend on the choice of $\psi$. We call $\mathbb{W}_{\psi}(g ; \mathbb{X})$ the $\mathcal{R}$-valued $\psi$-Whittaker function associated to the family $\left\{\mathbf{F}_{2 k^{\prime}+1}\right\}_{k^{\prime}}$. It is easily seen that $\left\{\mathbf{E}_{2 k^{\prime}+1, \chi}\right\}_{k^{\prime}}$ is an adelic compatible family of Eisenstein series.

Lemma 9.3. Let $\left\{\mathcal{F}_{2 k^{\prime}+1}\right\}_{k^{\prime}}$ be a family such that $\left\{\mathcal{F}_{2 k^{\prime}+1} \mid \gamma\right\}_{k^{\prime}}$ is a compatible family of Eisenstein series for any $\gamma \in \mathrm{SL}_{2}(\mathbb{Z})$. Then there exists an adelic compatible family $\left\{\mathbf{F}_{2 k^{\prime}+1}\right\}_{k^{\prime}}$ such that $\xi\left(\mathbf{F}_{2 k^{\prime}+1}\right)=\mathcal{F}_{2 k^{\prime}+1}$.

Proof. Put $\mathbf{F}_{2 k^{\prime}+1}=\xi^{-1}\left(\mathcal{F}_{2 k^{\prime}+1}\right)$. Let $W_{\psi, \mathbf{F}_{2 k^{\prime}+1}}^{0}$ be the finite part of the Whittaker function of $\mathbf{F}_{2 k^{\prime}+1}$. We have to prove that $W_{\psi, \mathbf{F}_{2 k^{\prime}+1}}^{0}$ is an $\mathcal{R}$-valued function, i.e. there exists an element $\varphi_{g}(\mathbb{X}) \in \mathcal{R}$ such that $W_{\psi, \mathbf{F}_{2 k^{\prime}+1}}^{0}(g)=\varphi_{g}\left(\left\{p^{-k^{\prime}}\right\}\right)$ for each $g \in \mathrm{GL}_{2}\left(\mathbb{A}_{\mathrm{f}}\right)$ and $k^{\prime} \geqslant k_{0}^{\prime}$. First assume that $g \in \mathrm{SL}_{2}\left(\mathbb{A}_{\mathrm{f}}\right)$. Then $\left\{\rho(g) \mathcal{F}_{2 k^{\prime}+1}\right\}_{k^{\prime}}$ is a compatible family of Eisenstein series, since $\mathrm{SL}_{2}(\mathbb{Q})=B(\mathbb{Q})$. $\mathrm{SL}_{2}(\mathbb{Z})$ (cf. the proof of [Ike01, Lemma 10.3]). Then $W_{\psi, \mathbf{F}_{2 k^{\prime}+1}}^{0}(g)=W_{\psi, \rho(g) \mathbf{F}_{2 k^{\prime}+1}}^{0}\left(\mathbf{1}_{2}\right)$ is essentially the $t$ th Fourier coefficient of $\rho\left(\mathbf{F}_{2 k^{\prime}+1}\right)$, where $t$ is the rational number such that $\psi(x)=\mathbf{e}_{\mathbb{A}}(t x)$. By Lemma 8.7(1), the restriction of $W_{\psi, \mathbf{F}_{2 k^{\prime}+1}}^{0}$ to $\mathrm{SL}_{2}\left(\mathbb{A}_{\mathrm{f}}\right)$ is an $\mathcal{R}$-valued $\psi$-Whittaker function. We shall show that $W_{\psi, \mathbf{F}_{2 k^{\prime}+1}}^{0}$ is an $\mathcal{R}$-valued function on $\mathrm{GL}_{2}\left(\mathbb{A}_{\mathrm{f}}\right)$. We may assume that $\mathbf{F}_{2 k^{\prime}+1}$ has an irreducible $\mathcal{K}$-type $u=\prod_{v} u_{v}$. By Lemma 8.6, $\left(\prod_{p} \beta_{\psi_{p}, u_{p}}(s)\right) W_{\psi, \mathbf{F}_{2 k^{\prime}+1}}^{0}$ is an $\mathcal{R}$-valued Whittaker function of $\tilde{I}(\mathbf{1} \otimes \underline{\chi}, s)$. By Lemma 8.3, there exists a non-trivial additive character $\psi^{\prime}$ such that the greatest common divisor of $\beta_{\psi_{p}, u_{p}}(s)$ and $\beta_{\psi_{p}^{\prime}, u_{p}}(s)$ is 1 for any $p$. It follows that $W_{\psi, \mathbf{F}_{2 k^{\prime}+1}}^{0}$ is an $\mathcal{R}$-valued Whittaker function. Hence the lemma is proved.

Proof of Lemma 7.4. Put $\mathbf{F}_{i, 2 k^{\prime}+1}=\xi^{-1}\left(\mathcal{F}_{i, 2 k^{\prime}+1}\right)$. Then, by Lemma 9.3, $\left\{\mathbf{F}_{i, 2 k^{\prime}+1}\right\}_{k^{\prime}}$ is an adelic compatible family. Let

$$
\overrightarrow{\mathbb{W}}(g, \mathbb{X})={ }^{\mathrm{t}}\left(\mathbb{W}_{1}(g, \mathbb{X}), \ldots, \mathbb{W}_{d}(g, \mathbb{X})\right)
$$

be the $\mathcal{R}^{d}$-valued $\psi$-Whittaker function associated to $\left\{\overrightarrow{\mathbf{F}}_{2 k^{\prime}+1}\right\}_{k^{\prime}}$. Put $W_{i}(g)=\mathbb{W}_{i}\left(g_{\mathrm{f}},\left\{\alpha_{p}\right\}\right) W_{\psi, 2 k+1}^{\infty}$ $\left(g_{\infty}\right)$ for $g=g_{\mathrm{f}} g_{\infty}, g_{\mathrm{f}} \in \mathrm{GL}_{2}\left(\mathbb{A}_{\mathrm{f}}\right), g_{\infty} \in \mathrm{GL}_{2}(\mathbb{R})$. Note that $\mathbb{W}_{i}\left(g_{\mathrm{f}},\left\{\alpha_{p}\right\}\right)$ is a well-defined Whittaker 


\section{LIFTING OF HERMITIAN MODULAR FORMS}

function of $\bigotimes_{p}^{\prime} \tilde{I}\left(\mathbf{1} \otimes \underline{\chi}, s_{0, p}\right)$ by Lemma $8.7(2)$. Then

$$
\mathbf{h}_{i}(g)=\sum_{a \in \mathbb{Q}^{\times}} W_{i}\left(\left(\begin{array}{ll}
a & 0 \\
0 & 1
\end{array}\right) g\right) \in \mathcal{V}(\mathbf{f})
$$

for $i=1, \ldots, d$, since $\pi$ is an irreducible cuspidal automorphic representation. $\operatorname{Put} \overrightarrow{\mathbf{h}}={ }^{\mathrm{t}}\left(\mathbf{h}_{1}, \ldots, \mathbf{h}_{d}\right)$. Then we have $\vec{h}=\xi(\overrightarrow{\mathbf{h}}) \in \mathcal{I}\left(\mathcal{V}(f)^{d} ; u\right)$.

\section{Proofs of Theorems 5.1 and 5.2}

Now we can prove Theorem 5.1. Let $k^{\prime}$ be a sufficiently large integer. Then there is a vector-valued modular form of weight $2 k^{\prime}+1$ with values in $\mathbb{C}^{\Xi}=\operatorname{Map}(\Xi, \mathbb{C})$ whose $\xi$ th component is the $(S, \xi)$ component of the Fourier-Jacobi coefficient of the Eisenstein series

$$
b_{S, \xi, 2 k^{\prime}+1}+\sum_{N \in \mathbb{Z}_{+}} N^{k^{\prime}}\left[\prod_{p \mid N} \tilde{F}_{p}\left(H_{S, \xi}(N) ; p^{-k^{\prime}}\right)\right] q^{N / \Delta} .
$$

Here $b_{S, \xi, 2 k^{\prime}+1}$ is some rational number and

$$
\begin{aligned}
\Delta & =\left[\left(\mathcal{O}^{\sharp}\right)^{m-1}: S \mathcal{O}^{m-1}\right]=D^{m-1}(\operatorname{det} S)^{2}, \\
H_{S, \xi}(N) & =\left(\begin{array}{cc}
{ }_{\mathrm{t}} \frac{S}{\xi} S & N / \Delta+{ }^{\mathrm{t}} \bar{\xi} S \xi
\end{array}\right) .
\end{aligned}
$$

As we have seen in $\S 6$, this vector-valued automorphic form is of type $u_{S}$. Note that the type $u_{S}$ does not depend on $k^{\prime}$.

By [Ike94, Theorem 3.2], $\mathcal{E}_{S, \xi}$ belongs to the space $\mathcal{V}\left(E_{2 k^{\prime}+1, \chi}\right)$ for each sufficiently large integer $k^{\prime}$. It follows that, when $k^{\prime}$ extends over sufficiently large integers, $\left(\mathcal{E}_{k^{\prime}+n}^{(2 n)}\right)_{S, \xi}(\tau)$ make up a compatible family of Eisenstein series. By Lemma 7.4,

$$
\sum_{N=1}^{\infty} N^{k}\left(\prod_{p \mid N} \tilde{F}_{p}\left(H_{S, \xi}(N) ; \alpha_{p}\right)\right) q^{N / \Delta}
$$

is a vector-valued automorphic form with type $u_{S}$. It follows that

$$
\sum_{\xi \in \Xi} \theta_{[\xi]}(S ; \tau, z) \sum_{N=1}^{\infty} N^{k}\left(\prod_{p \mid N} \tilde{F}_{p}\left(H_{S, \xi}(N) ; \alpha_{p}\right)\right) q^{N / \Delta}
$$

is a Jacobi form with index $S$. For

$$
\gamma=\left(\begin{array}{ll}
a & b \\
c & d
\end{array}\right)
$$

we put

$$
\bar{\gamma}=\left(\begin{array}{cccc}
\mathbf{1}_{m-1} & 0 & \mathbf{0}_{m-1} & 0 \\
0 & a & 0 & b \\
\mathbf{0}_{m-1} & 0 & \mathbf{1}_{m-1} & 0 \\
0 & c & 0 & d
\end{array}\right) .
$$

Then we have $\left.F(Z)\right|_{2 k+2 n} \bar{\gamma}=F(Z)$ for any $\gamma \in \mathrm{SL}_{2}(\mathbb{Z})$. It is proved by Klingen [Kli56] that $\Gamma_{K}^{(m)}$ is generated by $\Gamma_{K, \infty}^{(m)}$ and

$$
\left(\begin{array}{cc}
\mathbf{0}_{m} & -\mathbf{1}_{m} \\
\mathbf{1}_{m} & \mathbf{0}_{m}
\end{array}\right)
$$




\section{T. IKEDA}

(see also $\S 12$ ). Therefore $\left.F\right|_{2 k+2 n} g=(\operatorname{det} g)^{-k-n} F$ for any $g \in \Gamma_{K}^{(m)}$. This completes the proof of Theorem 5.1.

Now we consider Case O. Since the proof for Case O is almost the same as for Case E, we just describe an outline. Let

$$
E_{2 k}(\tau)=-\frac{B_{2 k}}{4 k}+\sum_{N=1}^{\infty}\left(\sum_{d \mid N} d^{2 k-1}\right) q^{N} \in M_{2 k}\left(\mathrm{SL}_{2}(\mathbb{Z})\right)
$$

be the usual Eisenstein series. Note that the Satake parameter of $E_{2 k}$ is $\left\{p^{k-(1 / 2)}, p^{-k+(1 / 2)}\right\}$.

Definition 10.1 (Case O). We define a compatible family of Eisenstein series $\left\{\mathcal{F}_{2 k^{\prime}}(\tau)\right\}_{k^{\prime}} \geqslant k_{0}^{\prime}$ as follows. A compatible family of Eisenstein series is a family $\left\{\mathcal{F}_{2 k^{\prime}}(\tau)\right\}_{k^{\prime}}$ of modular forms

$$
\mathcal{F}_{2 k^{\prime}}(\tau)=b\left(2 k^{\prime} ; 0\right)+\sum_{N \in \mathbb{Q}_{+}^{\times}} N^{k^{\prime}-(1 / 2)} b\left(2 k^{\prime} ; N\right) q^{N}
$$

satisfying the following conditions (1), (2), and (3):

(1) $\mathcal{F}_{2 k^{\prime}} \in \mathcal{V}\left(E_{2 k^{\prime}}\right)$ for any integer $k^{\prime} \geqslant k_{0}^{\prime}$;

(2) for each $N \in \mathbb{Q}_{+}^{\times}$, there exists an element $\Phi_{N}(\mathbb{X}) \in \mathcal{R}$ such that

$$
b\left(2 k^{\prime} ; N\right)=\Phi_{N}\left(\left\{p^{-k^{\prime}+(1 / 2)}\right\}\right) ;
$$

(3) there exists a congruence subgroup $\Gamma \subset \mathrm{SL}_{2}(\mathbb{Z})$ such that $\mathcal{F}_{2 k^{\prime}} \in M_{2 k^{\prime}}(\Gamma)$ for all $k^{\prime} \geqslant k_{0}^{\prime}$.

As in Case E, one can prove the following lemma.

Lemma 10.2. Let

$$
f(\tau)=\sum_{N>0} a(N) q^{N} \in S_{2 k}\left(\mathrm{SL}_{2}(\mathbb{Z})\right)
$$

be a normalized Hecke eigenform and $\alpha_{p}$ a Satake parameter of $f(\tau)$. Assume that there are a finitedimensional representation $\left(u, \mathbb{C}^{d}\right)$ of $\mathcal{K}$, and $\vec{\Phi}_{N}(\mathbb{X})={ }^{t}\left(\Phi_{1, N}(\mathbb{X}), \ldots, \Phi_{d, N}(\mathbb{X})\right) \in \mathcal{R}^{d}\left(N \in \mathbb{Q}_{+}^{\times}\right)$ satisfying the following conditions (1) and (2).

(1) For each sufficiently large integer $k^{\prime}$, there exists a vector-valued modular form

$$
\overrightarrow{\mathcal{F}}_{2 k^{\prime}}(\tau)=\vec{b}\left(2 k^{\prime} ; 0\right)+\sum_{N \in \mathbb{Q}_{+}^{\times}} N^{k^{\prime}-(1 / 2)} \vec{b}\left(2 k^{\prime} ; N\right) q^{N}
$$

of type $u$.

(2) For each $i(1 \leqslant i \leqslant d)$, the ith component $\mathcal{F}_{i, 2 k^{\prime}}(\tau)$ of $\overrightarrow{\mathcal{F}}_{2 k^{\prime}}(\tau)$ is a compatible family of Eisenstein series such that

$$
b_{i}\left(2 k^{\prime} ; N\right)=\Phi_{i, N}\left(\left\{p^{-k^{\prime}+(1 / 2)}\right\}\right) .
$$

Here $\vec{b}\left(2 k^{\prime} ; N\right)={ }^{\mathrm{t}}\left(b_{1}\left(2 k^{\prime} ; N\right), \ldots, b_{d}\left(2 k^{\prime} ; N\right)\right)$.

Put

$$
\vec{h}(\tau)=\sum_{N \in \mathbb{Q}_{+}^{\times}} N^{k-(1 / 2)} \vec{\Phi}_{N}\left(\left\{\alpha_{p}\right\}\right) q^{N} .
$$

Then we have $\vec{h}(\tau) \in \mathcal{I}\left(\mathcal{V}(f)^{d} ; u\right)$.

The proofs of Lemma 10.2 and Theorem 5.2 are the same as in Case E. 


\section{LIFTING OF HERMITIAN MODULAR FORMS}

\section{Existence of some hermitian matrices}

Lemma 11.1. If $m$ is divisible by 4 , then there exists an element $H_{1} \in \Lambda_{m}(\mathcal{O})^{+}$such that $\gamma\left(H_{1}\right)=1$. Proof. We may assume that $m=4$. We define hermitian matrices $H_{s}$ and $H_{d}$ by

$$
H_{s}=\sqrt{-D}^{-1}\left(\begin{array}{cc}
0 & -\mathbf{1}_{2} \\
\mathbf{1}_{2} & 0
\end{array}\right), \quad H_{d}=\left(\begin{array}{cc}
D^{-2} & 0 \\
0 & \mathbf{1}_{3}
\end{array}\right) .
$$

Then $H_{s} \in \Lambda_{4}(\mathcal{O}), H_{d}>0$ and $\operatorname{det} H_{s}=\operatorname{det} H_{d}$. Since $\operatorname{det} H_{s}=\operatorname{det} H_{d}$, there exists an element $X_{p} \in \mathrm{GL}_{4}\left(K_{p}\right)$ such that $H_{s}=X_{p} H_{d}{ }^{\mathrm{t}} \bar{X}_{p}$ for each prime $p$ (cf. Scharlau [Sch85]). Replacing $X_{p}$ by

$$
X_{p}\left(\begin{array}{cc}
\operatorname{det} X_{p}^{-1} & 0 \\
0 & \mathbf{1}_{3}
\end{array}\right)
$$

we may assume that $X_{p} \in \mathrm{SL}_{4}\left(K_{p}\right)$. We may also assume that $X_{p} \in \mathrm{SL}_{4}\left(\mathcal{O}_{p}\right)$ for almost all $p$. By the strong approximation theorem for $\mathrm{SL}_{4}$, there exists an element $X \in \mathrm{SL}_{4}(K)$ such that $X^{-1} X_{p} \in \mathrm{SL}_{4}\left(\mathcal{O}_{p}\right)$ for any $p$. Put $H_{1}=X H_{d}{ }^{t} \bar{X}$. Then we have $H_{1} \in \Lambda_{4}(\mathcal{O})^{+}$and $\gamma\left(H_{1}\right)=1$.

Lemma 11.2. Assume that $m$ is odd. For any integer $N>0$, there exists an element $H_{N} \in \Lambda_{m}(\mathcal{O})^{+}$ such that $\left|\gamma\left(H_{N}\right)\right|=N$.

Proof. By Lemma 11.1, we may assume that $m=1$ or 3 . If $m=1$, we put $H_{N}=(N)$. The proof in the case $m=3$ is similar to Lemma 11.1. One can take

$$
H_{s}=\left(\begin{array}{ccc}
-N & 0 & 0 \\
0 & 0 & -\sqrt{-D}^{-1} \\
0 & \sqrt{-D}^{-1} & 0
\end{array}\right), \quad H_{d}=\left(\begin{array}{cc}
N D^{-1} & 0 \\
0 & \mathbf{1}_{2}
\end{array}\right) .
$$

Then one can find a desired element $H_{N} \in \Lambda_{m}(\mathcal{O})^{+}$as in Lemma 11.1.

Lemma 11.3. Assume that $m=2 n$ is even. Let $N>0$ be a rational integer such that there exists an element $y \in \mathcal{O}^{\sharp}$ such that $D y \bar{y} \equiv(-1)^{n} N \bmod D$. Then there exists an element $H_{N} \in \Lambda_{m}(\mathcal{O})^{+}$ such that $\left|\gamma\left(H_{N}\right)\right|=N$.

Proof. We may assume that $m=2$ or 4 . Put $x=\left(D y \bar{y}-(-1)^{n} N\right) / D$. In the case $m=2$, one can put

$$
H_{N}=\left(\begin{array}{cc}
x & y \\
\bar{y} & 1
\end{array}\right)
$$

In the case $m=4$, we put

$$
H_{s}=\left(\begin{array}{cccc}
x & y & 0 & 0 \\
\bar{y} & 1 & 0 & 0 \\
0 & 0 & 0 & \sqrt{-D}^{-1} \\
0 & 0 & -\sqrt{-D}^{-1} & 0
\end{array}\right), \quad H_{d}=\left(\begin{array}{cc}
N D^{-2} & 0 \\
0 & \mathbf{1}_{3}
\end{array}\right) .
$$

Then one can find a desired element $H_{N} \in \Lambda_{m}(\mathcal{O})^{+}$as in Lemma 11.1.

LEMma 11.4. If $m=2 n$ is even, then there are an infinite number of primes $p$ such that $|\gamma(H)|=p$ for some $H \in \Lambda_{m}(\mathcal{O})^{+}$.

Proof. If $p \equiv(-1)^{n} \bmod D$, then there exists $H \in \Lambda_{m}(\mathcal{O})$ such that $|\gamma(H)|=p$ by Lemma 11.3. Hence the lemma is proved.

\section{Hermitian modular groups of general type}

In this section, we prove some facts about hermitian modular groups. We follow the argument of Klingen [Kli56]. 


\section{T. IKEDA}

For a fractional ideal $\mathfrak{a}$ of $K$, the absolute norm of $\mathfrak{a}$ is denoted by $\mathrm{N}(\mathfrak{a})$. For an integral ideal $\beta$ of $K$, the set of prime divisors of $\beta$ is denoted by $\operatorname{Supp}(\beta)$.

Lemma 12.1. Let $\mathfrak{a}$ be a fractional ideal and $\mathfrak{b}$ an integral ideal of $K$. Let $a \in \mathfrak{a}^{-1}$ and $b \in \mathcal{O}$ be elements such that $(a \mathfrak{a}, \mathfrak{b}, b)=1$. Then there exists an element $x \in \mathfrak{a}^{-1}$ such that $((a+x b) \mathfrak{a}, \mathfrak{b})=1$.

Proof. Decompose the integral ideals $a \mathfrak{a}$ and $\mathfrak{b}$ into products of integral ideals

$$
a \mathfrak{a}=\beta_{1} \gamma_{1}, \quad \mathfrak{b}=\beta_{2} \gamma_{2}, \quad\left(\beta_{1}, \gamma_{1}\right)=\left(\beta_{2}, \gamma_{2}\right)=\left(\gamma_{1}, \gamma_{2}\right)=1
$$

such that $\operatorname{Supp}\left(\beta_{1}\right)=\operatorname{Supp}\left(\beta_{2}\right)$. Let $\gamma_{3}$ be an integral ideal that belongs to the same ideal class as $\mathfrak{a} \gamma_{2}^{-1}$, such that $\left(\gamma_{3}, \beta_{1} \gamma_{1}\right)=1$. Let $x$ be an element such that $(x)=\mathfrak{a}^{-1} \gamma_{2} \gamma_{3}$. Assume that there is a prime ideal $\mathfrak{p}$ such that $\mathfrak{p} \mid((a+x b) \mathfrak{a}, \mathfrak{b})$. Then $\mathfrak{p} \mid \beta_{2}$ or $\mathfrak{p} \mid \gamma_{2}$. If $\mathfrak{p} \mid \beta_{2}$, then $\mathfrak{p} \mid \beta_{1}$, which is impossible, since $\left(\beta_{1}, x \mathfrak{a}\right)=\left(\beta_{1}, \gamma_{2} \gamma_{3}\right)=1$ and $\left(\beta_{1}, b\right)=1$. Now assume that $\mathfrak{p} \mid \gamma_{2}$. This is also impossible, since $\mathfrak{p} \mid x b \mathfrak{a}$ and $\mathfrak{p} \nmid a \mathfrak{a}$. Hence the lemma is proved.

Lemma 12.2. Let $\alpha, \mathfrak{a}_{1}, \mathfrak{a}_{2}$, and $\mathfrak{m}$ be fractional ideals of $K$. Then there exist elements $y_{1}, y_{2} \in K$ such that $y_{1} \in \alpha, \mathfrak{m}=y_{1} \mathfrak{a}_{1}+y_{2} \mathfrak{a}_{2}$.

Proof. Choose a non-zero element $y_{1} \in \mathfrak{m} \mathfrak{a}_{1}^{-1} \cap \alpha$. Put $\mathfrak{n}=y_{1} \mathfrak{a}_{1} \mathfrak{m}^{-1}$. Choose an integral ideal $\mathfrak{b}$ that belongs to the same ideal class as $\mathfrak{m}^{-1} \mathfrak{a}_{2}$, such that $(\mathfrak{b}, \mathfrak{n})=1$. Put $\left(y_{2}\right)=\mathfrak{m a}_{2}^{-1} \mathfrak{b}$. Then we have $y_{1} \mathfrak{a}_{1}+y_{2} \mathfrak{a}_{2}=\mathfrak{m} \mathfrak{n}+\mathfrak{m} \mathfrak{b}=\mathfrak{m}$.

We fix fractional ideals $\mathfrak{a}_{1}, \ldots, \mathfrak{a}_{m}$ of $\mathcal{O}$. We define an $\mathcal{O}$-module $\mathfrak{M}$ by

$$
\mathfrak{M}=\left\{{ }^{\mathrm{t}}\left(a_{1}, \ldots, a_{m}\right) \in K^{m} \mid a_{i} \in \mathfrak{a}_{i}(i=1, \ldots, m)\right\} .
$$

Note that the isomorphism class of $\mathfrak{M}$ is determined by the ideal class of $\mathfrak{a}_{1} \cdots \mathfrak{a}_{m}$. We define a group $\mathcal{U}$ by

$$
\mathcal{U}=\left\{g \in \mathrm{GL}_{m}(K) \mid g \mathfrak{M}=\mathfrak{M}\right\}
$$

Lemma 12.3. Let $\mathbf{x}={ }^{\mathrm{t}}\left(x_{1}, \ldots, x_{m}\right)$ and $\mathbf{y}={ }^{\mathrm{t}}\left(y_{1}, \ldots, y_{m}\right)$ be column vectors in $K^{m}$. Then there exists an element $g \in \mathcal{U}$ such that $g \mathbf{x}=\mathbf{y}$ if and only if $x_{1} \mathfrak{a}_{1}^{-1}+\cdots+x_{m} \mathfrak{a}_{m}^{-1}=y_{1} \mathfrak{a}_{1}^{-1}+\cdots+y_{m} \mathfrak{a}_{m}^{-1}$.

Proof. The 'only if' part is clear from the definition. We may assume that $m>1$. Assume that $x_{1} \mathfrak{a}_{1}^{-1}+\cdots+x_{m} \mathfrak{a}_{m}^{-1}=y_{1} \mathfrak{a}_{1}^{-1}+\cdots+y_{m} \mathfrak{a}_{m}^{-1} \neq\{0\}$. Let $G$ be the algebraic group defined by $G=$ $\left\{g \in \mathrm{SL}_{m}(K) \mid g \mathbf{x}=\mathbf{x}\right\}$. Then the strong approximation theorem holds for $G \simeq K^{m-1} \rtimes \mathrm{SL}_{m-1}(K)$. Put $\mathfrak{K}_{\mathfrak{p}}=\left\{g \in \mathrm{GL}_{m}\left(K_{\mathfrak{p}}\right) \mid g \mathfrak{M}_{\mathfrak{p}}=\mathfrak{M}_{\mathfrak{p}}\right\}$ for each prime $\mathfrak{p}$ of $K$. Here, $\mathfrak{M}_{\mathfrak{p}}$ is the closure of $\mathfrak{M}$ in $K_{\mathfrak{p}}^{m}$. Then $\prod_{\mathfrak{p}} \mathfrak{K}_{\mathfrak{p}} \times \mathrm{GL}_{m}(\mathbb{C})$ is an open subgroup of $\mathrm{GL}_{m}\left(\mathbb{A}_{K}\right)$. Choose an element $h \in \mathrm{GL}_{m}(K)$ such that $h \mathbf{x}=\mathbf{y}$. The set

$$
h^{-1}\left(\prod_{\mathfrak{p}} \mathfrak{K}_{\mathfrak{p}} \times \mathrm{GL}_{m}(\mathbb{C})\right) \cap G\left(\mathbb{A}_{K}\right)
$$

is a non-empty open subset of $G\left(\mathbb{A}_{K}\right)$ by our assumption. By the strong approximation theorem for $G$, there exists an element

$$
g \in h^{-1}\left(\prod_{\mathfrak{p}} \mathfrak{K}_{\mathfrak{p}} \times \mathrm{GL}_{m}(\mathbb{C})\right) \cap G(K) .
$$

Then we have $h g \in \mathcal{U}$ and $h g \mathbf{x}=\mathbf{y}$.

We define a group $\boldsymbol{\Gamma}$ by

$$
\boldsymbol{\Gamma}=\left\{g \in \mathrm{U}(m, m)(\mathbb{Q}) \mid g\left(\begin{array}{c}
\mathfrak{M} \\
\mathfrak{M}^{\prime}
\end{array}\right)=\left(\begin{array}{c}
\mathfrak{M} \\
\mathfrak{M}^{\prime}
\end{array}\right)\right\},
$$




\section{LIFTING OF HERMITIAN MODULAR FORMS}

where $\mathfrak{M}^{\prime}=\left\{{ }^{\mathrm{t}}\left(a_{1}, \ldots, a_{m}\right) \in K^{m} \mid a_{i} \in \overline{\mathfrak{a}}_{i}^{-1}(i=1, \ldots, m)\right\}$. We temporarily call $\boldsymbol{\Gamma}$ a hermitian modular group of general type associated to $\mathfrak{M}$. If $\mathfrak{M}_{1}$ and $\mathfrak{M}_{2}$ are isomorphic $\mathcal{O}$-modules, then the corresponding groups $\boldsymbol{\Gamma}_{1}$ and $\boldsymbol{\Gamma}_{2}$ are conjugate by an element of $\mathrm{U}(m, m)(\mathbb{Q})$. We define the subgroups $M_{\Gamma}, N_{\Gamma}$, and $\tilde{N}_{\Gamma}$ of $\Gamma$ by

$$
\begin{aligned}
& M_{\boldsymbol{\Gamma}}=\left\{\left(\begin{array}{cc}
g & 0 \\
0 & { }^{\mathrm{t}} \bar{g}^{-1}
\end{array}\right) \mid g \in \mathcal{U}\right\}, \\
& N_{\boldsymbol{\Gamma}}=\left\{\left(\begin{array}{cc}
\mathbf{1}_{m} & B \\
0 & \mathbf{1}_{m}
\end{array}\right) \mid{ }^{\mathrm{t}} \bar{B}=B=\left(b_{i j}\right), b_{i j} \in \mathfrak{a}_{i} \overline{\mathfrak{a}}_{j}\right\}, \\
& \tilde{N}_{\boldsymbol{\Gamma}}=\left\{\left(\begin{array}{cc}
\mathbf{1}_{m} & 0 \\
B & \mathbf{1}_{m}
\end{array}\right) \mid{ }^{\mathrm{t}} \bar{B}=B=\left(b_{i j}\right), b_{i j} \in \overline{\mathfrak{a}}_{i}^{-1} \mathfrak{a}_{j}^{-1}\right\} .
\end{aligned}
$$

Let us denote the subgroup generated by $M_{\Gamma}, N_{\boldsymbol{\Gamma}}$, and $\tilde{N}_{\boldsymbol{\Gamma}}$ by $\boldsymbol{\Gamma}^{\prime}$. We shall prove that $\boldsymbol{\Gamma}^{\prime}=\boldsymbol{\Gamma}$. To prove this, we may assume that $\mathfrak{a}_{1}=\mathcal{O}$, by replacing $\mathfrak{a}_{i}$ by $\mathfrak{a}_{1}^{-1} \mathfrak{a}_{i}$, and $\boldsymbol{\Gamma}$ by

$$
\left(\begin{array}{cc}
\mathbf{1}_{m} & 0 \\
0 & \mathrm{~N}\left(\mathfrak{a}_{1}\right) \mathbf{1}_{m}
\end{array}\right) \boldsymbol{\Gamma}\left(\begin{array}{cc}
\mathbf{1}_{m} & 0 \\
0 & \mathrm{~N}\left(\mathfrak{a}_{1}\right)^{-1} \mathbf{1}_{m}
\end{array}\right) \text {. }
$$

Since it is easy to prove that $\boldsymbol{\Gamma}^{\prime}=\boldsymbol{\Gamma}$ for $m=1$, we consider the case $m \geqslant 2$.

Lemma 12.4. Assume that $m \geqslant 2$ and that $\mathfrak{a}_{1}=\mathcal{O}$. Let $b_{1}, \ldots, b_{m}, c_{1}, \ldots, c_{m}$ be elements of $K$ satisfying the following properties (1) and (2):

(1) $\sum_{i=1}^{m} b_{i} \mathfrak{a}_{i}^{-1}+c_{i} \overline{\mathfrak{a}}_{i}=\mathcal{O}$;

(2) $b_{1} \bar{c}_{1}+\cdots+b_{m} \bar{c}_{m} \in \mathbb{Z}$.

Put $\mathbf{e}_{1}={ }^{\mathrm{t}}(1,0, \ldots, 0) \in K^{2 m}$. Then there exists an element $g \in \Gamma^{\prime}$ such that

$$
g \mathbf{e}_{1}={ }^{\mathrm{t}}\left(b_{1}, \ldots, b_{m}, c_{1}, \ldots, c_{m}\right) .
$$

Proof. Note that $b_{1}, c_{1} \in \mathcal{O}$, since $\mathfrak{a}_{1}=\mathcal{O}$. Note also that the properties (1) and (2) are preserved by changing ${ }^{\mathrm{t}}\left(b_{1}, \ldots, b_{m}, c_{1}, \ldots, c_{m}\right)$ by $g \cdot{ }^{\mathrm{t}}\left(b_{1}, \ldots, b_{m}, c_{1}, \ldots, c_{m}\right)$ with $g \in \boldsymbol{\Gamma}$. By using Lemmas 12.2 and 12.3, we may assume that $c_{3}=\cdots=c_{m}=0$. If $c_{1}=c_{2}=0$, then ${ }^{\mathrm{t}}\left(b_{1}, \ldots, b_{m}\right)$ is the first row of an element of $\mathcal{U}$ by Lemma 12.3 , in which case we are done. Assume that $\left(c_{1}, c_{2}\right) \neq(0,0)$. Put $\alpha=\sum_{i=3}^{m} b_{i} \mathfrak{a}_{i}^{-1}, \alpha^{\prime}=\sum_{i=1}^{m} b_{i} \mathfrak{a}_{i}^{-1}$. By Lemmas 12.2 and 12.3 , we may assume that

$$
c_{1} c_{2} \neq 0, \quad \alpha^{\prime} \mid c_{2} \overline{\mathfrak{a}}_{2} .
$$

If $b_{1}=0$, we replace $b_{1}$ by $b_{1}+c_{1}$. Thus we may assume that

$$
\left(b_{1}, b_{1} \mathfrak{a}_{2}^{-1}, \alpha, c_{1}\right)=1, \quad b_{1} c_{1} c_{2} \neq 0, \quad c_{3}=\cdots=c_{m}=0, \quad \alpha^{\prime} \mid c_{2} \overline{\mathfrak{a}}_{2} .
$$

Let $\rho$ be the product of all prime ideals $\mathfrak{p}$ such that $\overline{\mathfrak{p}} \mid b_{1}, \mathfrak{p} \nmid b_{1}$. Then we have $\left(b_{2} \mathfrak{a}_{2}^{-1}, \alpha, \rho, b_{1}\right)=1$. By Lemma 12.1, there exists $x \in \mathfrak{a}_{2}$ such that $\left(\left(b_{2}+b_{1} x\right) \mathfrak{a}_{2}^{-1}, \alpha, \rho\right)=1$. Note that $\left(b_{1},\left(b_{2}+\right.\right.$ $\left.\left.b_{1} x\right) \mathfrak{a}_{2}^{-1}, \alpha, c_{1}-\bar{x} c_{2}\right)=1$. Decompose the integral ideals $\left(\left(b_{2}+b_{1} x\right) \mathfrak{a}_{2}^{-1}, \alpha\right)$ and $b_{1}$ into products of integral ideals

$$
\begin{aligned}
\left(\left(b_{2}+b_{1} x\right) \mathfrak{a}_{2}^{-1}, \alpha\right) & =\beta_{1} \gamma_{1}, \quad b_{1}=\beta_{2} \gamma_{2}, \\
\left(\beta_{1}, \gamma_{1}\right) & =\left(\beta_{2}, \gamma_{2}\right)=\left(\gamma_{1}, \gamma_{2}\right)=1,
\end{aligned}
$$

such that $\operatorname{Supp}\left(\beta_{1}\right)=\operatorname{Supp}\left(\beta_{2}\right)$. Put $y=\mathrm{N}\left(\gamma_{1}\right)$. We shall show that

$$
\left(b_{1}+y\left(c_{1}-\bar{x} c_{2}\right),\left(b_{2}+b_{1} x\right) \mathfrak{a}_{2}^{-1}, \alpha\right)=1 .
$$

Let $\mathfrak{p}$ be a prime divisor of the left-hand side. Then we have either $\mathfrak{p} \mid \beta_{1}$ or $\mathfrak{p} \mid \gamma_{1}$. If $\mathfrak{p} \mid \gamma_{1}$, then we have $\mathfrak{p} \mid y$, and so $\mathfrak{p} \mid b_{1}$, which is impossible. Now we assume that $\mathfrak{p} \mid \beta_{1}$. Then we have $\mathfrak{p} \mid \beta_{2}$, and so $\mathfrak{p} \mid b_{1}$. It follows that $\mathfrak{p} \mid y\left(c_{1}-\bar{x} c_{2}\right)$. Note that $\mathfrak{p} \mid\left(c_{1}-\bar{x} c_{2}\right)$ contradicts $\left(b_{1},\left(b_{2}+b_{1} x\right) \mathfrak{a}_{2}^{-1}, \alpha, c_{1}-\bar{x} c_{2}\right)=1$. 


\section{T. IKEDA}

If $\mathfrak{p} \mid y$, then we have $\mathfrak{p} \mid \bar{\gamma}_{1}$, since $\mathfrak{p} \mid \gamma_{1} \bar{\gamma}_{1}$ and $\mathfrak{p} \mid \beta_{1}$. This implies that $\overline{\mathfrak{p}} \nmid b_{1}$, and so $\overline{\mathfrak{p}} \mid \rho$, which contradicts to $\left(\left(b_{2}+b_{1} x\right) \mathfrak{a}_{2}^{-1}, \alpha, \rho\right)=1$. Hence we have $\left(b_{1}+y\left(c_{1}-\bar{x} c_{2}\right),\left(b_{2}+b_{1} x\right) \mathfrak{a}_{2}^{-1}, \alpha\right)=1$. We replace ${ }^{\mathrm{t}}\left(b_{1}, \ldots, b_{m}, c_{1}, \ldots, c_{m}\right)$ by

$$
\left(\begin{array}{lll|lll}
1 & 0 & 0 & 0 & 0 & 0 \\
0 & 1 & 0 & 0 & 0 & 0 \\
0 & 0 & \mathbf{1}_{m^{\prime}} & 0 & 0 & 0 \\
\hline y & 0 & 0 & 1 & 0 & 0 \\
0 & 0 & 0 & 0 & 1 & 0 \\
0 & 0 & 0 & 0 & 0 & \mathbf{1}_{m^{\prime}}
\end{array}\right)\left(\begin{array}{lll|lll}
1 & 0 & 0 & 0 & 0 & 0 \\
x & 1 & 0 & 0 & 0 & 0 \\
0 & 0 & \mathbf{1}_{m^{\prime}} & 0 & 0 & 0 \\
\hline 0 & 0 & 0 & 1 & -\bar{x} & 0 \\
0 & 0 & 0 & 0 & 1 & 0 \\
0 & 0 & 0 & 0 & 0 & \mathbf{1}_{m^{\prime}}
\end{array}\right)\left(\begin{array}{c}
b_{1} \\
b_{2} \\
\vdots \\
c_{1} \\
c_{2} \\
\vdots
\end{array}\right)
$$

where $m^{\prime}=m-2$. Then we have $b_{1} \mathfrak{a}_{1}^{-1}+b_{2} \mathfrak{a}_{2}^{-1}+\cdots+b_{m} \mathfrak{a}_{m}=\mathcal{O}$. In this case, the proposition can be easily proved by using Lemma 12.3 .

Now we prove that $\boldsymbol{\Gamma}^{\prime}=\boldsymbol{\Gamma}$ by induction with respect to $m$. Let $g$ be any element of $\boldsymbol{\Gamma}$ and put ${ }^{\mathrm{t}}\left(b_{1}, \ldots, b_{m}, c_{1}, \ldots, c_{m}\right)=g \mathbf{e}_{1}$. Then it is easy to see that $b_{1}, \ldots, b_{m}, c_{1}, \ldots, c_{m}$ satisfy the properties (1) and (2) of Lemma 12.4. By Lemma 12.4, there exists an element $h \in \Gamma^{\prime}$ such that $g \mathbf{e}_{1}=h \mathbf{e}_{1}$. Put

$$
h^{-1} g=\left(\begin{array}{cc|cc}
1 & * & * & * \\
0 & A & * & B \\
\hline 0 & 0 & 1 & 0 \\
0 & C & * & D
\end{array}\right), \quad g_{1}=\left(\begin{array}{cc|cc}
1 & 0 & 0 & 0 \\
0 & A & 0 & B \\
\hline 0 & 0 & 1 & 0 \\
0 & C & 0 & D
\end{array}\right) .
$$

By the induction hypothesis, we have $g_{1} \in \Gamma^{\prime}$. Clearly, we have $g_{1}^{-1} h^{-1} g \in \Gamma^{\prime}$. Thus we have $g \in h g_{1} \boldsymbol{\Gamma}^{\prime}=\boldsymbol{\Gamma}^{\prime}$.

Proposition 12.5. Put $a_{i}=\mathrm{N}\left(\mathfrak{a}_{i}\right)(i=1, \ldots, m)$. The hermitian modular group $\boldsymbol{\Gamma}$ is generated by $M_{\Gamma} N_{\Gamma}$ and

$$
w_{j}=\left(\begin{array}{lll|lll}
\mathbf{1}_{j-1} & 0 & 0 & 0 & 0 & 0 \\
0 & 0 & 0 & 0 & a_{j} & 0 \\
0 & 0 & \mathbf{1}_{m-j} & 0 & 0 & 0 \\
\hline 0 & 0 & 0 & \mathbf{1}_{j-1} & 0 & 0 \\
0 & a_{j}^{-1} & 0 & 0 & 0 & 0 \\
0 & 0 & 0 & 0 & 0 & \mathbf{1}_{m-j}
\end{array}\right), \quad 1 \leqslant j \leqslant m .
$$

Proof. Put $A=\operatorname{diag}\left(a_{1}, \ldots, a_{m}\right)$. Then we have

$$
\left(\begin{array}{cc}
0 & A \\
A^{-1} & 0
\end{array}\right) N_{\Gamma}\left(\begin{array}{cc}
0 & A \\
A^{-1} & 0
\end{array}\right)=\tilde{N}_{\Gamma}
$$

Thus the proposition follows from Lemma 12.4 .

Proposition 12.6. Let $P$ be the Siegel parabolic subgroup of $\mathcal{G}=\mathrm{U}(m, m)$. Then we have $\mathcal{G}(\mathbb{Q})=$ $P(\mathbb{Q}) \cdot \boldsymbol{\Gamma}$.

Proof. Put $\mathcal{G}_{1}=\mathrm{SU}(m, m), P_{1}=P \cap \mathcal{G}_{1}$, and $\boldsymbol{\Gamma}_{1}=\boldsymbol{\Gamma} \cap \mathcal{G}_{1}(\mathbb{Q})$. It is enough to prove that $\mathcal{G}_{1}(\mathbb{Q})=$ $P_{1}(\mathbb{Q}) \cdot \boldsymbol{\Gamma}_{1}$.

Let $\mathcal{K}_{1}$ be the maximal compact subgroup of $\mathcal{G}_{1}\left(\mathbb{A}_{\mathrm{f}}\right)$ defined by $\mathcal{K}_{1}=\mathcal{G}_{1}\left(\mathbb{A}_{\mathrm{f}}\right) \cap\left(\prod_{p} \mathrm{GL}_{2 m}\left(\mathcal{O}_{p}\right)\right)$. For $j=1, \ldots, m$, we choose elements $t_{j} \in \mathbb{A}_{K}^{\times}$such that $\operatorname{ord}_{\mathfrak{p}} t_{j}=\operatorname{ord}_{\mathfrak{p}} \mathfrak{a}_{j},\left(t_{j}\right)_{\infty}=1$. Put

$$
\begin{aligned}
t & =\operatorname{diag}\left(t_{1}, \ldots, t_{m}, \bar{t}_{i}^{-1}, \ldots, \bar{t}_{m}^{-1}\right) \in \mathcal{G}\left(\mathbb{A}_{\mathbf{f}}\right), \\
\mathcal{K}_{\boldsymbol{\Gamma}} & =t \mathcal{K}_{1} t^{-1} .
\end{aligned}
$$




\section{LIFTING OF HERMITIAN MODULAR FORMS}

Then we have $\mathcal{G}_{1}(\mathbb{Q}) \cap \mathcal{K}_{\boldsymbol{\Gamma}} \mathcal{G}_{1}(\mathbb{R})=\boldsymbol{\Gamma}_{1}$. Note that the Levi factor of $P_{1}$ is isomorphic to $\{m \in$ $\left.\mathrm{GL}_{m}(K) \mid \operatorname{det} m \in \mathbb{Q}^{\times}\right\}$. In particular, the class number of $P_{1}$ is 1 . Thus we have

$$
P_{1}(\mathbb{Q}) \mathcal{K}_{\Gamma} \mathcal{G}_{1}(\mathbb{R})=P_{1}(\mathbb{A}) \mathcal{K}_{\Gamma} \mathcal{G}_{1}(\mathbb{R})=\mathcal{G}_{1}(\mathbb{A}) .
$$

Hence we have

$$
\mathcal{G}_{1}(\mathbb{Q})=P_{1}(\mathbb{Q}) \cdot\left(\mathcal{G}_{1}(\mathbb{Q}) \cap \mathcal{K}_{\Gamma} \mathcal{G}_{1}(\mathbb{R})\right)=P_{1}(\mathbb{Q}) \cdot \Gamma_{1} .
$$

Hence the proposition is proved.

\section{Extension to the unitary group}

In this section, we discuss the extension of $\operatorname{Lift}^{(m)}(f)$ to an automorphic form on the adele group of the unitary group $\mathcal{G}=\mathrm{U}(m, m)$. Since $\mathcal{G}$ need not have class number 1 , we need to consider several congruence subgroups. Let $T$ be the maximal torus of $\mathcal{G}$, which consists of all diagonal elements. Let $\mathcal{K}$ be the maximal compact subgroup of $\mathcal{G}\left(\mathbb{A}_{\mathrm{f}}\right)$ defined by $\mathcal{K}=\mathcal{G}\left(\mathbb{A}_{\mathrm{f}}\right) \cap\left(\prod_{p} \mathrm{GL}_{2 m}\left(\mathcal{O}_{p}\right)\right)$.

Lemma 13.1. There is a natural bijection between the double coset $\mathcal{G}(\mathbb{Q}) \backslash \mathcal{G}(\mathbb{A}) / \mathcal{K}(\mathbb{R})$ and the ideal class group $\mathcal{C}_{K}$ of $K$.

Proof. Put $H=\mathrm{U}(1)$. Then by strong approximation of $\mathrm{SU}(m, m)$, there is a bijection $\mathcal{G}(\mathbb{Q}) \backslash \mathcal{G}(\mathbb{A}) /$ $\mathcal{K G}(\mathbb{R}) \rightarrow H(\mathbb{Q}) \backslash H(\mathbb{A}) / \mathcal{I} H(\mathbb{R})$, where $\mathcal{I}$ is the image of $\mathcal{K}$ in $H\left(\mathbb{A}_{\mathrm{f}}\right)$. Consider the exact sequence

$$
1 \rightarrow \mathbb{A}_{\mathbb{Q}}^{\times} \rightarrow \mathbb{A}_{K}^{\times} \rightarrow H(\mathbb{A}) \rightarrow 1,
$$

where the map $\mathbb{A}_{K}^{\times} \rightarrow H(\mathbb{A})$ is given by $x \mapsto x \bar{x}^{-1}$. One can easily show that $\mathcal{I} \subset H\left(\mathbb{A}_{\mathrm{f}}\right)$ is equal to the image of $\prod_{\mathfrak{p}} \mathcal{O}_{\mathfrak{p}}^{\times} \subset \mathbb{A}_{K, \mathrm{f}}^{\times}$. Then we have $H(\mathbb{Q}) \backslash H(\mathbb{A}) / \mathcal{I} H(\mathbb{R}) \simeq \mathbb{A}_{K}^{\times} / \mathbb{A}_{\mathbb{Q}}^{\times} \prod_{\mathfrak{p}} \mathcal{O}_{\mathfrak{p}}^{\times} \mathbb{C}^{\times} \simeq \mathcal{C}_{K}$.

Fix a complete set of representatives $\left\{\gamma_{1}, \ldots, \gamma_{h}\right\}$ for the double coset $\mathcal{G}(\mathbb{Q}) \backslash \mathcal{G}(\mathbb{A}) / \mathcal{K} \mathcal{G}(\mathbb{R})$. We may assume that $\gamma_{1}=\mathbf{1}_{2 m}$ and

$$
\gamma_{i}=\left(\begin{array}{cc}
t_{i} & 0 \\
0 & \bar{t}_{i}^{-1}
\end{array}\right) \in T\left(\mathbb{A}_{\mathrm{f}}\right)
$$

Let $\mathfrak{c}_{i}$ be the ideal of $K$ such that $\operatorname{ord}_{\mathfrak{p}}\left(\mathfrak{c}_{i}\right)=\operatorname{ord}_{\mathfrak{p}}\left(\left(\operatorname{det} t_{i}\right)_{\mathfrak{p}}\right)$, where $\operatorname{det} t_{i}$ is considered as an element of $\mathbb{A}_{K}^{\times}$. Then the bijection $\mathcal{G}(\mathbb{Q}) \backslash \mathcal{G}(\mathbb{A}) / \mathcal{K G}(\mathbb{R}) \rightarrow \mathcal{C}_{K}$ is given by

$$
\mathcal{G}(\mathbb{Q}) \gamma_{i} \mathcal{K G}(\mathbb{R}) \mapsto \text { the ideal class of } \mathfrak{c}_{i}
$$

Put

$$
\Gamma_{i}=\Gamma_{i}^{(m)}=\mathcal{G}(\mathbb{Q}) \cap\left(\gamma_{i} \mathcal{K} \gamma_{i}^{-1} \cdot \mathcal{G}(\mathbb{R})\right)
$$

Then $\Gamma_{i}$ is a hermitian modular group of general type considered in $\S 12$. Put

$$
\Lambda_{m}(\mathcal{O})_{i}=\left\{H \in \mathcal{H}_{m}(K) \mid \bar{t}_{i, p} H t_{i, p} \in \Lambda_{m}\left(\mathcal{O}_{p}\right) \text { for any } p\right\} .
$$

The set of positive definite elements of $\Lambda_{m}(\mathcal{O})_{i}$ is denoted by $\Lambda_{m}(\mathcal{O})_{i}^{+}$.

For a holomorphic function $F$ on $\mathcal{H}_{m}$ and $g \in \mathcal{G}(\mathbb{R})$, we introduce the notation

$$
F \|_{2 l} g=\left.(\operatorname{det} g)^{l} \cdot F\right|_{2 l} g \text {. }
$$

When $l$ is clear from the context, we drop it from the notation. For $m \geqslant 2$, put

$$
M_{2 l}\left(\Gamma_{i}, \operatorname{det}^{-l}\right)=\left\{F \mid F \|_{2 l} g=F \text { for any } g \in \Gamma_{i}\right\} .
$$

For $m=1$, we require the usual holomorphy condition at cusps. Then $F \in M_{2 l}\left(\Gamma_{i}\right.$, det $\left.{ }^{-l}\right)$ has a Fourier expansion of the form

$$
F(Z)=\sum_{\substack{H \in \Lambda_{m}(\mathcal{O})_{i} \\ H \geqslant 0}} A(H) \mathbf{e}(H Z)
$$




\section{T. IKEDA}

The space of cusp forms $S_{2 l}\left(\Gamma_{i}, \operatorname{det}^{-l}\right)$ is defined by

$$
\left\{F \in M_{2 l}\left(\Gamma_{i}, \operatorname{det}^{-l}\right) \mid A(H)=0 \text { unless } H \in \Lambda_{m}(\mathcal{O})_{i}^{+}\right\} .
$$

Put $\mathbf{i}=\sqrt{-1} \cdot \mathbf{1}_{m} \in \mathcal{H}_{m}$. For $\left(F_{1}, \ldots, F_{h}\right) \in \bigoplus_{i=1}^{h} M_{2 l}\left(\Gamma_{i}, \operatorname{det}^{-l}\right)$, we put

$$
\left(F_{1}, \ldots, F_{h}\right)^{\sharp}(g)=\left(F_{i} \|_{2 l} x\right)(\mathbf{i})=F_{i}(x\langle\mathbf{i}\rangle) j(x, \mathbf{i})^{-2 l}(\operatorname{det} x)^{l}
$$

for $g=u \gamma_{i} x \kappa, u \in \mathcal{G}(\mathbb{Q}), x \in \mathcal{G}(\mathbb{R}), \kappa \in \mathcal{K}$. Then $\left(F_{1}, \ldots, F_{h}\right)^{\sharp}$ is an automorphic form on $\mathcal{G}(\mathbb{A})$. We denote by $\mathcal{M}_{2 l}\left(\mathcal{G}(\mathbb{Q}) \backslash \mathcal{G}(\mathbb{A})\right.$, det $\left.^{-l}\right)$ the space of automorphic forms obtained in this way. Similarly, we put $\mathcal{S}_{2 l}\left(\mathcal{G}(\mathbb{Q}) \backslash \mathcal{G}(\mathbb{A})\right.$, $\left.\operatorname{det}^{-l}\right)=\left\{\left(F_{1}, \ldots, F_{h}\right)^{\sharp} \mid F_{i} \in S_{2 l}\left(\Gamma_{i}, \operatorname{det}^{-l}\right)\right\}$.

Let $\mathbf{f}_{2 l}$ be the function on $\mathcal{G}(\mathbb{A})$ defined by

$$
\mathbf{f}_{2 l}(g)=\prod_{p}\left|\operatorname{det}\left(d_{p} \bar{d}_{p}\right)\right|_{p}^{-l} j\left(g_{\infty}, \mathbf{i}\right)^{-2 l}\left(\operatorname{det} g_{\infty}\right)^{l},
$$

where $g=\left(g_{v}\right)_{v} \in \mathcal{G}(\mathbb{A})$,

$$
g_{p}=\left(\begin{array}{cc}
a_{p} & b_{p} \\
0 & d_{p}
\end{array}\right) \kappa_{p}, \quad\left(\begin{array}{cc}
a_{p} & b_{p} \\
0 & d_{p}
\end{array}\right) \in P\left(\mathbb{Q}_{p}\right),
$$

and $\kappa_{p} \in \mathcal{K}_{p}$. We consider the normalized Eisenstein series

$$
\mathbf{E}_{2 l}^{(m)}(g)=2^{-m} \prod_{i=1}^{m} L\left(i-2 l, \chi^{i-1}\right) \sum_{\gamma \in P(\mathbb{Q}) \backslash \mathcal{G}(\mathbb{Q})} \mathbf{f}_{2 l}(\gamma g) .
$$

Then we have $\mathbf{E}_{2 l}^{(m)} \in \mathcal{M}_{2 l}\left(\mathcal{G}(\mathbb{Q}) \backslash \mathcal{G}(\mathbb{A}), \operatorname{det}^{-l}\right)$. We denote the corresponding Eisenstein series for $\Gamma_{i}$ by $\mathcal{E}_{i, 2 l}$. Thus $\left(\mathcal{E}_{i, 2 l}, \ldots, \mathcal{E}_{i, 2 l}\right)^{\sharp}=\mathbf{E}_{2 l}^{(m)}$ and

$$
\mathcal{E}_{i, 2 l}(Z)=2^{-m} \prod_{i=1}^{m} L\left(i-2 l, \chi^{i-1}\right) \sum_{g \in \Gamma_{i, \infty} \backslash \Gamma_{i}}(\operatorname{det} g)^{l} j(g, Z)^{-2 l},
$$

where $\Gamma_{i, \infty}=P(\mathbb{Q}) \cap \Gamma_{i}$. Note that $P(\mathbb{Q}) \backslash \mathcal{G}(\mathbb{Q}) \simeq \Gamma_{i, \infty} \backslash \Gamma_{i}$ by Proposition 12.6. As in $\S 4$, one can show that the $H$ th Fourier coefficient of $\mathcal{E}_{i, 2 l}(Z)$ is equal to

$$
|\gamma(H)|^{l-(m / 2)} \prod_{p}\left|\operatorname{det} t_{i, p} \bar{t}_{i, p}\right|_{p}^{m / 2} \tilde{F}_{p}\left(\bar{t}_{i, p} H t_{i, p} ; p^{-l+(m / 2)}\right)
$$

for any $H \in \Lambda_{m}(\mathcal{O})_{i}^{+}$and any sufficiently large integer $l$. Set

$$
\Phi_{i}(H, \mathbb{X})=\prod_{p}\left|\operatorname{det} t_{i, p} \bar{t}_{i, p}\right|_{p}^{m / 2} \tilde{F}_{p}\left(\bar{t}_{i, p} H t_{i, p} ; X_{p}\right) \in \mathcal{R}
$$

Then, for sufficiently large $k^{\prime}$, the $H$ th Fourier coefficient of $\mathcal{E}_{i, 2 k^{\prime}+2 n}$ is equal to $|\gamma(H)|^{k^{\prime}-(\mathfrak{e} / 2)} \Phi_{i}(H$, $\left.\left\{p^{-k^{\prime}+(\mathfrak{e} / 2)}\right\}\right)$, where $n=[m / 2], \mathfrak{e}=m-2 n$.

Theorem 13.2. In Case E, let $f(\tau) \in S_{2 k+1}\left(\Gamma_{0}(D), \chi\right)$ be a primitive form with Satake parameter $\left\{\alpha_{p}, \beta_{p}\right\}$. Then, for $i=1, \ldots, h$, the Fourier series

$$
F_{i}(Z)=\sum_{H \in \Lambda_{2 n}(\mathcal{O})_{i}^{+}}|\gamma(H)|^{k} \Phi_{i}\left(H,\left\{\alpha_{p}\right\}\right) \mathbf{e}(H Z)
$$

belongs to $S_{2 k+2 n}\left(\Gamma_{i}^{(2 n)}, \operatorname{det}^{-k-n}\right)$. Put $\mathcal{F}=\left(F_{1}, \ldots, F_{h}\right)^{\sharp}$. Then $\mathcal{F}$ is independent of the choice of the representatives $\left\{\gamma_{1}, \ldots, \gamma_{h}\right\}$. 


\section{LIFTING OF HERMITIAN MODULAR FORMS}

Theorem 13.3. In Case $O$, let $f(\tau) \in S_{2 k}\left(\mathrm{SL}_{2}(\mathbb{Z})\right)$ be a normalized Hecke eigenform with Satake parameter $\left\{\alpha_{p}, \alpha_{p}^{-1}\right\}$. Then, for $i=1, \ldots, h$, the Fourier series

$$
F_{i}(Z)=\sum_{H \in \Lambda_{2 n+1}(\mathcal{O})_{i}^{+}}|\gamma(H)|^{k-(1 / 2)} \Phi_{i}\left(H,\left\{\alpha_{p}\right\}\right) \mathbf{e}(H Z)
$$

belongs to $S_{2 k+2 n}\left(\Gamma_{i}^{(2 n+1)}, \operatorname{det}^{-k-n}\right)$. Put $\mathcal{F}=\left(F_{i}, \ldots, F_{i}\right)^{\sharp}$. Then $\mathcal{F}$ is independent of the choice of the representatives $\left\{\gamma_{1}, \ldots, \gamma_{h}\right\}$.

We prove only Theorem 13.3. The proof of Theorem 13.2 can be treated in the same way.

Proof. By Proposition 12.5, $\boldsymbol{\Gamma}=\Gamma_{i}$ is generated by $M_{\boldsymbol{\Gamma}} N_{\boldsymbol{\Gamma}}$ and $w_{j}(j=1, \ldots, m)$, where $M_{\boldsymbol{\Gamma}}, N_{\boldsymbol{\Gamma}}$ and $w_{j}$ are as in $\S 12$. Clearly, $F_{i}(Z)$ is modular with respect to $M_{\Gamma} N_{\Gamma}$. As in $\S 10$, one can prove that $F_{i}(Z)$ is modular with respect to $w_{m}$. By permutation of coordinates, $F_{i}(Z)$ is also modular with respect to $w_{j}(j=1, \ldots, m)$. Therefore $F_{i}(Z)$ is a hermitian modular form with respect to $\Gamma_{i}$. The modularity of $F_{i}$ follows from this. That $F_{i}$ is a cusp form follows from Proposition 12.6.

We prove that the definition of $\mathcal{F}$ is independent of the choice of $\left\{\gamma_{1}, \ldots, \gamma_{h}\right\}$. Let $M$ be the Levi factor of the parabolic subgroup $P$ such that

$$
M(\mathbb{Q})=\left\{\left(\begin{array}{cc}
A & 0 \\
0 & { }^{\mathrm{t}} \bar{A}^{-1}
\end{array}\right) \mid A \in \mathrm{GL}_{m}(K)\right\} .
$$

Then the natural map

$$
M(\mathbb{Q}) \backslash M(\mathbb{A}) /\left(\mathcal{K} \cap M\left(\mathbb{A}_{\mathrm{f}}\right)\right) M(\mathbb{R}) \rightarrow \mathcal{G}(\mathbb{Q}) \backslash \mathcal{G}(\mathbb{A}) / \mathcal{K} \mathcal{G}(\mathbb{R})
$$

is surjective. Let $\left\{\gamma_{1}^{\prime}, \ldots, \gamma_{h}^{\prime}\right\}$,

$$
\gamma_{i}^{\prime}=\left(\begin{array}{cc}
t_{i}^{\prime} & 0 \\
0 & \overline{t_{i}^{\prime}}-1
\end{array}\right) \in T\left(\mathbb{A}_{\mathrm{f}}\right)
$$

be another set of representatives. Then there exists $u_{i} \in M(\mathbb{Q})$ such that $t_{i}^{\prime} \in u_{i} t_{i}\left(\mathcal{K} \cap M\left(\mathbb{A}_{\mathrm{f}}\right)\right) M(\mathbb{R})$. By multiplying some element in $\mathcal{K}$ from the right, we may assume that $t_{i}^{\prime}=u_{i, \mathrm{f}} t_{i}$, where $u_{i}=u_{i, \mathrm{f}} u_{i, \infty}$, $u_{i, \mathrm{f}} \in M\left(\mathbb{A}_{\mathrm{f}}\right), u_{i, \infty} \in M(\mathbb{R})$. Note that

$$
\gamma_{i} x=\left(\begin{array}{cc}
u_{i}^{-1} & 0 \\
0 & { }^{\mathrm{t}} \bar{u}_{i}
\end{array}\right) \gamma_{i}^{\prime}\left(\begin{array}{cc}
u_{i, \infty} & 0 \\
0 & { }^{\mathrm{t}} \bar{u}_{i, \infty}^{-1}
\end{array}\right) x
$$

for $x \in \mathcal{G}(\mathbb{R})$. We define $\mathcal{E}_{i, 2 l}^{\prime}$ and $F_{i}^{\prime}$ by using the representative $\gamma_{i}^{\prime}$ instead of $\gamma_{i}$. Then we have

$$
\left(\mathcal{E}_{i, 2 l} \|_{2 l} x\right)(\mathbf{i})=\mathbf{E}_{2 l}^{(m)}\left(\gamma_{i} x\right)=\left(\mathcal{E}_{i, 2 l}^{\prime} \|_{2 l}\left(\begin{array}{cc}
u_{i, \infty} & 0 \\
0 & { }^{\mathrm{t}} \bar{u}_{i, \infty}^{-1}
\end{array}\right) x\right)(\mathbf{i})
$$

It follows that

$$
\mathcal{E}_{i, 2 l}^{\prime}=\mathcal{E}_{i, 2 l} \|_{2 l}\left(\begin{array}{cc}
u_{i}^{-1} & 0 \\
0 & { }^{\mathrm{t}} \bar{u}_{i}
\end{array}\right)
$$

Comparing the Fourier expansion, we have

$$
F_{i}^{\prime}=F_{i} \|_{2 k+2 n}\left(\begin{array}{cc}
u_{i}^{-1} & 0 \\
0 & { }^{\mathrm{t}} \bar{u}_{i}
\end{array}\right)
$$

which implies the desired independence.

Definition 13.4. We call $\mathcal{F}$ in Theorem 13.2 or Theorem 13.3 the lift of $f(\tau)$ to $\mathcal{S}_{2 k+2 n}(\mathcal{G}(\mathbb{Q}) \backslash \mathcal{G}(\mathbb{A})$, $\left.\operatorname{det}^{-k-n}\right)$ and denote it by Lift $^{(m)}(f)$. 


\section{T. IKEDA}

Next, we shall show that Lift ${ }^{(m)}(f)$ is a common Hecke eigenform for all Hecke operators if it is not identically zero. Recall that the Eisenstein series $\mathbf{E}_{2 l}^{(m)}$ is a common Hecke eigenform for all Hecke operators for $\left(\mathcal{G}\left(\mathbb{A}_{\mathrm{f}}\right), \mathcal{K}\right)$.

Recall that the action of a Hecke operator can be described as follows. Let $T=\mathcal{K}_{q} \mathcal{K}$ be a double coset for $q \in \mathcal{G}\left(\mathbb{A}_{\mathrm{f}}\right)$. Let

$$
\mathcal{K} q \mathcal{K}=\coprod_{r \in I} \mathcal{K} q_{r}, \quad q_{r} \in \mathcal{G}\left(\mathbb{A}_{\mathrm{f}}\right)
$$

be a decomposition into a sum of left cosets, where $I$ is a finite index set. For each $r \in I$, and $j \in\{1, \ldots, h\}$, choose $i_{r, j} \in\{1, \ldots, h\}$ and $u_{r, j} \in \mathcal{G}(\mathbb{Q})$ such that $\gamma_{j} q_{r} \in u_{r, j} \gamma_{i_{r, j}} \mathcal{K} \mathcal{G}(\mathbb{R})$. Then the action of the Hecke operator $T$ on $\mathcal{S}_{2 l}\left(\mathcal{G}(\mathbb{Q}) \backslash \mathcal{G}(\mathbb{A})\right.$, det $^{-l}$ ) is given by

$$
\left(F_{1}, \ldots, F_{h}\right)^{\sharp} \mid T=\left(\sum_{r \in I} F_{i_{r, 1}}\left\|_{2 l} u_{r, 1}^{-1}, \ldots, \sum_{r \in I} F_{i_{r, h}}\right\|_{2 l} u_{r, h}^{-1}\right)^{\sharp} .
$$

Note that one can assume that $u_{r, i} \in P(\mathbb{Q})$ by Proposition 12.6.

Proposition 13.5. For each Hecke operator $T$ for $\left(\mathcal{G}\left(\mathbb{A}_{\mathrm{f}}\right), \mathcal{K}\right)$, there exists an element $\Phi_{T} \in \mathcal{R}$ such that

$$
\mathbf{E}_{2 k^{\prime}+2 n}^{(m)} \mid T=\Phi_{T}\left(\left\{p^{-k^{\prime}+(\mathfrak{e} / 2)}\right\}\right) \mathbf{E}_{2 k^{\prime}+2 n}^{(m)}
$$

where $\mathfrak{e}=0$ in Case $E$ and $\mathfrak{e}=1$ in Case $O$.

Proof. Put $\mathbf{E}_{2 k^{\prime}+2 n}^{(m)} \mid T=\left(E_{1, T}, \ldots, E_{h, T}\right)$. Then, by using Proposition 12.6, one can easily show that $E_{i, T}$ has a Fourier expansion

$$
E_{i, T}(Z)=\sum_{H \in \Lambda_{m}(\mathcal{O})_{i}^{+}}|\gamma(H)|^{k^{\prime}-(\mathfrak{e} / 2)} \Phi_{i, T}^{\prime}\left(H,\left\{p^{-k^{\prime}+(\mathfrak{e} / 2)}\right\}\right)
$$

for some $\Phi_{i, T}^{\prime}(\mathbb{X}) \in \mathcal{R}$ (cf. [Ike01, p. 664]). If $m \not \equiv 2 \bmod 4$, then there exists an element $H \in \Lambda_{m}(\mathcal{O})$ such that $|\gamma(H)|=1$ by Lemmas 11.1 and 11.2 . For such an $H$, we have $\tilde{F}_{p}(H, X)=1$ for any prime $p$. Therefore the eigenvalue is equal to $\Phi_{1, T}^{\prime}\left(H,\left\{p^{-k^{\prime}+(\mathfrak{e} / 2)}\right\}\right)$. If $m \equiv 2 \bmod 4$, there exist an infinite number of primes $p$ such that $p=-\gamma(H)$ for some $H \in \Lambda_{m}(\mathcal{O})^{+}$by Lemma 11.4. Choose such primes $p_{1} \neq p_{2}$ with $p_{1}=-\gamma\left(H_{1}\right)$ and $p_{2}=-\gamma\left(H_{2}\right)$. Note that $\operatorname{ord}_{p_{1}} F_{p_{1}}\left(H_{1}, X\right)=\operatorname{ord}_{p_{2}} F_{p_{2}}\left(H_{2}, X\right)=1$. By the functional equation, we have $F_{p_{1}}\left(H_{1}, X\right)=F_{p_{2}}\left(H_{2}, X\right)=1+X$. It follows that

$$
\prod_{p} \tilde{F}_{p}\left(H_{1}, X_{p}\right)=X_{p_{1}}+X_{p_{1}}^{-1}, \quad \prod_{p} \tilde{F}_{p}\left(H_{2}, X_{p}\right)=X_{p_{2}}+X_{p_{2}}^{-1} .
$$

By Lemma 7.1, we have

$$
\left(X_{p_{1}}+X_{p_{1}}^{-1}\right) \Phi_{1, T}^{\prime}\left(H_{2}, \mathbb{X}\right)=\left(X_{p_{2}}+X_{p_{2}}^{-1}\right) \Phi_{1, T}^{\prime}\left(H_{1}, \mathbb{X}\right)
$$

By unique factorization of $\mathcal{R}$, we have $\Phi_{1, T}^{\prime}\left(H_{1}, \mathbb{X}\right) /\left(X_{p_{1}}+X_{p_{1}}^{-1}\right) \in \mathcal{R}$. Hence the proposition is proved.

THEOREM 13.6. The automorphic form $\operatorname{Lift}^{(m)}(f)$ is a common eigenform of all Hecke operators for $\left(\mathcal{G}\left(\mathbb{A}_{\mathrm{f}}\right), \mathcal{K}\right)$. Moreover, for each Hecke operator $T$ for $\left(\mathcal{G}\left(\mathbb{A}_{\mathrm{f}}\right), \mathcal{K}\right)$, we have

$$
\text { Lift }^{(m)}(f) \mid T=\Phi_{T}\left(\left\{\alpha_{p}\right\}\right) \text { Lift }^{(m)}(f) .
$$

Proof. As in [Ike01, §11], the theorem follows from Proposition 13.5 and Lemma 7.1.

Let $\mathfrak{c}$ be an integral ideal of $K$. We assume that $\mathfrak{c}$ is prime to $D$. Set $C=\mathrm{N}(\mathfrak{c})$. We choose a finite idele $t=\left(t_{\mathfrak{p}}\right)_{\mathfrak{p}} \in \mathbb{A}_{K, \mathrm{f}}$ such that $\operatorname{ord}_{\mathfrak{p}} t_{\mathfrak{p}}=\operatorname{ord}_{\mathfrak{p}} \mathfrak{c}$ for each prime ideal $\mathfrak{p}$ of $K$. Set

$$
\mathbf{t}=\left(\begin{array}{cc}
\mathbf{1}_{m-1} & 0 \\
0 & t
\end{array}\right), \quad \gamma=\left(\begin{array}{cc}
\mathbf{t} & 0 \\
0 & \overline{\mathbf{t}}^{-1}
\end{array}\right) \text {. }
$$




\section{LIFTING OF HERMITIAN MODULAR FORMS}

Definition 13.7. We set

$$
\begin{aligned}
\Gamma_{K}^{(m)}[\mathfrak{c}] & =\mathcal{G}(\mathbb{Q}) \cap\left(\gamma \mathcal{K} \gamma^{-1} \cdot \mathcal{G}(\mathbb{R})\right), \\
\Lambda_{m}^{\mathfrak{c}}(\mathcal{O})^{+} & =\left\{H \in \mathcal{H}_{m}(K) \mid H>0, \overline{\mathbf{t}}_{p} H \mathbf{t}_{p} \in \Lambda_{m}\left(\mathcal{O}_{p}\right) \text { for any } p\right\} .
\end{aligned}
$$

If $f \in S_{2 k}\left(\mathrm{SL}_{2}(\mathbb{Z})\right)$ is a normalized Hecke eigenform, then the lift of $f$ in $S_{2 k+2 n}\left(\Gamma_{K}^{(2 n+1)}[\mathfrak{c}]\right.$, det $\left.{ }^{-k-n}\right)$ is denoted by $\operatorname{Lift}_{\mathfrak{c}}^{(2 n+1)}(f)$. Similarly, if $f \in S_{2 k+1}\left(\Gamma_{0}(D), \chi\right)$ is a primitive form, then the lift of $f$ in $S_{2 k+2 n}\left(\Gamma_{K}^{(2 n)}[\mathfrak{c}]\right.$, det $\left.{ }^{-k-n}\right)$ is denoted by $\operatorname{Lift}_{\mathfrak{c}}^{(2 n)}(f)$. When $\mathfrak{c}=\mathcal{O}$, we simply drop $\mathfrak{c}$ from the notation.

Note that $\Gamma_{K}^{(m)}[\mathfrak{c}]$ is a hermitian modular group of general type considered in $\S 12$. We have

$$
\operatorname{Lift}_{\mathfrak{c}}^{(m)}(f)=C^{-k-n} \sum_{H \in \Lambda_{m}^{\mathfrak{c}}(\mathcal{O})^{+}}|C \gamma(H)|^{k-(\mathfrak{e} / 2)} \prod_{p} \tilde{F}_{p}\left(\overline{\mathbf{t}}_{p} H \mathbf{t}_{p} ; \alpha_{p}\right) \mathbf{e}(H Z),
$$

where $\mathfrak{e}=m-2[m / 2]$. If $\mathfrak{c}_{1}=\mathcal{O}, \mathfrak{c}_{2}, \ldots, \mathfrak{c}_{h}$ is a representative for the ideal class group, then we have

$$
\operatorname{Lift}^{(m)}(f)=\left(\operatorname{Lift}^{(m)}(f), \operatorname{Lift}_{\mathfrak{c}_{2}}^{(m)}(f), \ldots, \operatorname{Lift}_{\mathfrak{c}_{h}}^{(m)}(f)\right)^{\sharp} .
$$

The proofs of the following lemmas are the same as for Lemmas 11.2 and 11.3. We omit the details.

Lemma 13.8. Assume that $m$ is odd. For any integer $N>0$, there exists an element $H_{N} \in \Lambda_{m}^{\mathfrak{c}}(\mathcal{O})^{+}$ such that $C\left|\gamma\left(H_{N}\right)\right|=N$.

Lemma 13.9. Assume that $m=2 n$ is even. Let $N>0$ be a rational integer such that there exists an element $y \in \mathfrak{c}^{-1} \mathcal{O}^{\sharp}$ such that $C D y \bar{y} \equiv(-1)^{n} N \bmod D$. Then there exists an element $H_{N} \in \Lambda_{m}^{\mathfrak{c}}(\mathcal{O})^{+}$ such that $C\left|\gamma\left(H_{N}\right)\right|=N$.

\section{Linearization of the lifting for Case $\mathrm{O}$}

In this section, we consider only Case O. Put $m=2 n+1$. We fix an integral ideal $\mathfrak{c}$ of $K$ such that $C=\mathrm{N}(\mathfrak{c})$ is prime to $D$. We choose $\mathbf{t}$ as in the last section. Let $f(\tau) \in S_{2 k}\left(\mathrm{SL}_{2}(\mathbb{Z})\right)$ be a normalized Hecke eigenform. For each $N>0$, we put

$$
\begin{aligned}
\Psi_{p}(N ; X) & =p^{-e / 2} \frac{X^{e+1}-X^{-e-1}}{X-X^{-1}}, \quad e=\operatorname{ord}_{p} N \\
\Psi(N ; \mathbb{X}) & =\prod_{p \mid N} \Psi_{p}\left(N ; X_{p}\right) \in \mathcal{R} .
\end{aligned}
$$

Then the $N$ th Fourier coefficient $a_{f}(N)$ of $f$ is equal to $N^{k} \Psi\left(N ;\left\{\alpha_{p}\right\}\right)$.

Fix an element $H \in \Lambda_{2 n+1}^{\mathfrak{c}}(\mathcal{O})^{+}$. Then $\tilde{F}_{p}\left(\overline{\mathbf{t}}_{p} H \mathbf{t}_{p} ; X\right)$ belongs to the $\mathbb{C}$-vector space

$$
\left\{\Phi \in X^{-\operatorname{ord}_{p} C \gamma(H)} \mathbb{C}\left[X^{2}\right] \mid \Phi\left(X^{-1}\right)=\Phi(X)\right\} .
$$

Note that

$$
\left\{\Psi_{p}\left(\frac{C|\gamma(H)|}{p^{2 i}} ; X\right) \mid 0 \leqslant 2 i \leqslant \operatorname{ord}_{p} C \gamma(H)\right\}
$$

is a basis of this vector space. It follows that there exists $\phi(a, H) \in \mathbb{C}$ for each $a^{2} \mid C \gamma(H)$ such that

$$
|C \gamma(H)|^{-1 / 2} \prod_{p \mid C \gamma(H)} \tilde{F}_{p}\left(\overline{\mathbf{t}}_{p} H \mathbf{t}_{p} ; X_{p}\right)=\sum_{a^{2} \mid C \gamma(H)} \phi(a, H) \Psi\left(\frac{C|\gamma(H)|}{a^{2}} ; \mathbb{X}\right) .
$$

One can easily show that $\phi(a, H) \in \mathbb{Q}$. Moreover, $\phi(1, H)=1$ for any $H \in \Lambda_{2 n+1}^{\mathfrak{c}}(\mathcal{O})^{+}$, as the constant term of $F_{p}\left(\overline{\mathbf{t}}_{p} H \mathbf{t}_{p} ; X\right)$ is 1 . 


\section{T. IKEDA}

For each $f_{0}(\tau)=\sum_{N>0} a_{f_{0}}(N) q^{N} \in S_{2 k}\left(\mathrm{SL}_{2}(\mathbb{Z})\right)$, we put

$$
\iota\left(f_{0}\right)(Z)=\sum_{H \in \Lambda_{2 n+1}^{c}(\mathcal{O})^{+}} \sum_{a^{2} \mid C \gamma(H)} a^{2 k} \phi(a, H) a_{f_{0}}\left(\frac{C|\gamma(H)|}{a^{2}}\right) \mathbf{e}(H Z) .
$$

If $f$ is a normalized Hecke eigenform, then $\iota(f)=C^{k+n} \operatorname{Lift}_{\mathfrak{c}}^{(2 n+1)}(f)$. Since normalized Hecke eigenforms span $S_{2 k}\left(\mathrm{SL}_{2}(\mathbb{Z})\right)$, the image of $\iota$ is contained in $S_{2 k+2 n}\left(\Gamma_{K}^{(2 n+1)}[\mathfrak{c}]\right.$, $\left.\operatorname{det}^{-k-n}\right)$.

We shall show that $\iota$ is injective. Assume that $\iota\left(f_{0}\right)=0$ for some $f_{0} \in S_{2 k}\left(\mathrm{SL}_{2}(\mathbb{Z})\right)$. We show that all Fourier coefficients of $f_{0}$ are 0. By Lemma 13.8, there exists an element $H_{N} \in \Lambda_{2 n+1}^{\mathfrak{c}}(\mathcal{O})^{+}$ such that $C\left|\gamma\left(H_{N}\right)\right|=N$. Then the $H_{N}$ th Fourier coefficient of $\iota\left(f_{0}\right)$ is $a_{f_{0}}(N)+$ (lower terms). It follows that $a_{f_{0}}(N)=0$ by induction. Thus we have proved the following theorem.

TheOREM 14.1. There exists an injective linear map

$$
\iota: S_{2 k}\left(\mathrm{SL}_{2}(\mathbb{Z})\right) \rightarrow S_{2 k+2 n}\left(\Gamma_{K}^{(2 n+1)}[\mathfrak{c}], \operatorname{det}^{-k-n}\right)
$$

satisfying the following properties:

(1) for each $f_{0}=\sum_{N>0} a_{f_{0}}(N) q^{N} \in S_{2 k}\left(\mathrm{SL}_{2}(\mathbb{Z})\right)$, we have

$$
\iota\left(f_{0}\right)(Z)=\sum_{H \in \Lambda_{2 n+1}^{c}(\mathcal{O})^{+}} \sum_{a^{2} \mid C \gamma(H)} a^{2 k} \phi(a, H) a_{f_{0}}\left(\frac{C|\gamma(H)|}{a^{2}}\right) \mathbf{e}(H Z) ;
$$

(2) if $f(\tau) \in S_{2 k}\left(\mathrm{SL}_{2}(\mathbb{Z})\right)$ is a normalized Hecke eigenform, then $\iota(f)=C^{k+n} \operatorname{Lift}_{\mathfrak{c}}^{(2 n+1)}(f)$.

Corollary 14.2. Let $f(\tau) \in S_{2 k}\left(\mathrm{SL}_{2}(\mathbb{Z})\right)$ be a normalized Hecke eigenform. Then $\operatorname{Lift}_{\mathfrak{c}}^{(2 n+1)}(f)$ is not identically zero.

Obviously, Corollary 14.2 implies that $\operatorname{Lift}^{(2 n+1)}(f) \neq 0$ for any normalized Hecke eigenform $f \in S_{2 k}\left(\mathrm{SL}_{2}(\mathbb{Z})\right)$.

\section{Linearization of the lifting for Case $\mathrm{E}$}

In this section, we consider Case E. Put $m=2 n$. We fix an integral ideal $\mathfrak{c}$ of $K$ such that $C=\mathrm{N}(\mathfrak{c})$ is prime to $D$. We are going to show that the lifting can be described by a linear map

$$
S_{2 k+1}^{\varepsilon *}\left(\Gamma_{0}(D), \chi\right) \rightarrow S_{2 k+2 n}\left(\Gamma_{K}^{(2 n)}[\mathfrak{c}], \operatorname{det}^{-k-n}\right),
$$

where $S_{2 k+1}^{\varepsilon *}\left(\Gamma_{0}(D), \chi\right)$ is a certain subspace of $S_{2 k+1}\left(\Gamma_{0}(D), \chi\right)$. Unlike Case O, the subspace $S_{2 k+1}^{\varepsilon *}$ $\left(\Gamma_{0}(D), \chi\right)$ depends on the ideal class (more precisely, the genus) of $\mathfrak{c}$.

Let $Q_{D}$ be the set of all primes that divide $D$. For each prime $q \in Q_{D}$, we put $D_{q}=q^{\operatorname{ord}_{q} D}$. We define a primitive Dirichlet character $\chi_{q}$ by

$$
\chi_{q}(N)= \begin{cases}\chi\left(N^{\prime}\right) & \text { if }(N, q)=1, \\ 0 & \text { if } q \mid N,\end{cases}
$$

where $N^{\prime}$ is an integer such that

$$
N^{\prime} \equiv \begin{cases}N & \bmod D_{q} \\ 1 & \bmod D_{q}^{-1} D\end{cases}
$$

Then we have $\chi=\prod_{q \mid D} \chi_{q}$. Note that $D_{q}$ is the conductor of $\chi_{q}$ and that $\chi_{q}$ corresponds to the quadratic field with discriminant $\chi_{q}(-1) D_{q}$. One should not confuse $\chi_{q}$ with $\underline{\chi}_{q}$.

LEMma 15.1. If $q \nmid N$, then $\chi_{q}(N)=\underline{\chi}_{q}(N)$. 


\section{LIFTING OF HERMITIAN MODULAR FORMS}

Proof. Since $\chi_{q}$ corresponds to $\mathbb{Q}\left(\sqrt{\chi_{q}(-1) D_{q}}\right) / \mathbb{Q}$, we have

$$
\begin{aligned}
\chi_{q}(N) & =\left(\frac{\chi_{q}(-1) D_{q}, N}{\mathbb{Q}_{q}}\right) \\
& =\left(\frac{-D, N}{\mathbb{Q}_{q}}\right) \times \prod_{\substack{q^{\prime} \in Q_{D} \\
q^{\prime} \neq q}}\left(\frac{\chi_{q^{\prime}}(-1) D_{q^{\prime}}, N}{\mathbb{Q}_{q}}\right) .
\end{aligned}
$$

In this equation, the second factor is trivial since both $\chi_{q^{\prime}}(-1) D_{q^{\prime}}$ and $N$ are units in $\mathbb{Q}_{q}$, and $\chi_{q^{\prime}}(-1) D_{q^{\prime}} \equiv 1 \bmod 4$ if $q=2$.

When $Q$ is a subset of $Q_{D}$, we set

$$
\chi_{Q}=\prod_{q \in Q} \chi_{q}, \quad \chi_{Q}^{\prime}=\prod_{\substack{q \in Q_{D} \\ q \notin Q}} \chi_{q}, \quad D_{Q}=\prod_{q \in Q} D_{q} .
$$

When $Q=\{q\}, \chi_{\{q\}}^{\prime}$ is simply denoted by $\chi_{q}^{\prime}$.

As in $\S 13$, let $\mathfrak{c}$ be an integral ideal of $K$ such that $C=\mathrm{N}(\mathfrak{c})$ is prime to $D$. Put $\varepsilon(Q)=\chi_{Q}(C)$. Then we have

$$
\begin{aligned}
\varepsilon(\emptyset) & =\varepsilon\left(Q_{D}\right)=1, \\
\varepsilon(Q) \varepsilon\left(Q^{\prime}\right) & =\varepsilon\left(Q \cup Q^{\prime}\right) \varepsilon\left(Q \cap Q^{\prime}\right),
\end{aligned}
$$

for any $Q, Q^{\prime} \subset Q_{D}$. By genus theory (see e.g. Hecke [Hec81, §48]), any function $\varepsilon:\{Q \mid Q \subset$ $\left.Q_{D}\right\} \rightarrow\{ \pm 1\}$ with these properties is obtained in this way. Moreover, two integral ideals $\mathfrak{c}$ and $\mathfrak{c}^{\prime}$ give the same function $\varepsilon$ if and only if $\mathfrak{c}$ and $\mathfrak{c}^{\prime}$ belong to the same genus. If $Q=\{q\}$, we denote $\varepsilon(\{q\})$ simply by $\varepsilon(q)$.

We fix a primitive form $f=\sum a_{f}(N) q^{N} \in S_{2 k+1}\left(\Gamma_{0}(D), \chi\right)$. Recall that, for each subset $Q \subset Q_{D}$, there exists a primitive form

$$
f_{Q}=\sum_{N>0} b(N) q^{N} \in S_{2 k+1}\left(\Gamma_{0}(D), \chi\right)
$$

such that (see Miyake [Miy89, Theorem 4.6.16])

$$
\begin{cases}b(p)=\chi_{Q}(p) a_{f}(p) & \text { if } p \notin Q, \\ b(q)=\chi_{Q}^{\prime}(q) \overline{a_{f}(q)} & \text { if } q \in Q .\end{cases}
$$

Note that $\left(f_{Q}\right)_{Q^{\prime}}=f_{Q^{\prime \prime}}$ where $Q^{\prime \prime}=\left(Q \cup Q^{\prime}\right)-\left(Q \cap Q^{\prime}\right)$.

Definition 15.2. If $f \in S_{2 k+1}\left(\Gamma_{0}(D), \chi\right)$ is a primitive form, we put

$$
f^{\varepsilon *}=\sum_{Q \subset Q_{D}} \varepsilon(Q) \chi_{Q}(-1)^{n} f_{Q} .
$$

When $\varepsilon=1, f^{\varepsilon *}$ is simply denoted by $f^{*}$.

Obviously, $\left(f_{Q}\right)^{\varepsilon *}=\varepsilon(Q) \chi_{Q}(-1)^{n} f^{\varepsilon *}$.

Definition 15.3. Following Krieg [Kri91], we define

$$
\mathbf{a}_{D}^{\varepsilon}(N)=\prod_{q \in Q_{D}}\left(1+\varepsilon(q) \chi_{q}\left((-1)^{n} N\right)\right)=\prod_{\substack{q \in Q_{D} \\ q \nmid N}}\left(1+\underline{\chi}_{q}\left((-1)^{n} C N\right)\right) .
$$

Here, $\varepsilon(q)=\varepsilon(\{q\})$. When $\varepsilon=1, \mathbf{a}_{D}^{\varepsilon}(N)$ is simply denoted by $\mathbf{a}_{D}(N)$. Note that $\mathbf{a}_{D}^{\varepsilon}(N)=\mathbf{a}_{D}(C N)$. 


\section{T. IKEDA}

Lemma 15.4. The N th Fourier coefficient of $f_{Q}$ is equal to

$$
a_{f_{Q}}(N)=a_{f}\left(N^{\prime} N_{Q}^{\prime}\right) \overline{a_{f}\left(N_{Q}\right)} \prod_{q \in Q} \underline{\chi}_{q}(N),
$$

where

$$
N^{\prime}=\prod_{p \nmid D} p^{\operatorname{ord}_{p} N}, \quad N_{Q}^{\prime}=\prod_{\substack{q \in Q_{D} \\ q \notin Q}} q^{\operatorname{ord}_{q} N}, \quad N_{Q}=\prod_{q \in Q} q^{\operatorname{ord}_{q} N} .
$$

Proof. It is enough to consider the case when $Q=\{q\}$ and $q \mid N$. In this case, we have

$$
\begin{aligned}
a_{f_{Q}}(N) & =a_{f_{Q}}\left(N^{\prime}\right) a_{f_{Q}}\left(N_{Q}^{\prime}\right) a_{f_{Q}}\left(N_{Q}\right) \\
& =a_{f}\left(N^{\prime} N_{Q}^{\prime}\right) \overline{a_{f}\left(N_{Q}\right)} \chi_{q}\left(N^{\prime} N_{Q}^{\prime}\right) \chi_{q}^{\prime}\left(N_{Q}\right) .
\end{aligned}
$$

By Lemma 15.1, we have $\chi_{q}\left(N^{\prime} N_{Q}^{\prime}\right)=\underline{\chi}_{q}\left(N^{\prime} N_{Q}^{\prime}\right)$. We shall show that

$$
\chi_{q}^{\prime}\left(N_{Q}\right)=\left(\frac{-D, N_{Q}}{\mathbb{Q}_{q}}\right)=\underline{\chi}_{q}\left(N_{Q}\right) .
$$

It is enough to show that $\chi_{q}^{\prime}(q)=\underline{\chi}_{q}(q)$, since $N_{Q}$ is a power of $q$. By Lemma 15.1, we have

$$
\chi_{q}^{\prime}(q)=\prod_{\substack{q^{\prime} \in Q_{D} \\ q^{\prime} \neq q}} \chi_{q^{\prime}}(q)=\prod_{\substack{q^{\prime} \in Q_{D} \\ q^{\prime} \neq q}}\left(\frac{-D, q}{\mathbb{Q}_{q^{\prime}}}\right) .
$$

By the Hilbert product formula,

$$
\chi_{q}^{\prime}(q) \underline{\chi}_{q}(q)=\left(\frac{-D, q}{\mathbb{R}}\right) \prod_{p \notin Q_{D}}\left(\frac{-D, q}{\mathbb{Q}_{p}}\right) .
$$

The first factor on the right-hand side is 1 since $q>0$. The second factor is 1 because both $-D$ and $q$ are units in $\mathbb{Q}_{p}$, and $-D \equiv 1 \bmod 4$ if $2 \notin Q_{D}$. Hence the lemma is proved.

Corollary 15.5. The Nth Fourier coefficient of $f^{\varepsilon *}$ is given by

$$
\begin{aligned}
a_{f^{\varepsilon *}}(N) & =a_{f}\left(N^{\prime}\right) \prod_{q \mid D}\left(a_{f}\left(N_{q}\right)+\underline{\chi}_{q}\left((-1)^{n} C N\right) \overline{a_{f}\left(N_{q}\right)}\right) \\
& =\mathbf{a}_{D}^{\varepsilon}(N) a_{f}\left(N^{\prime}\right) \prod_{q \mid(D, N)}\left(a_{f}\left(N_{q}\right)+\underline{\chi}_{q}\left((-1)^{n} C N\right) \overline{a_{f}\left(N_{q}\right)}\right) .
\end{aligned}
$$

Here $N_{q}=N_{\{q\}}$. In particular, we have $a_{f^{\varepsilon *}}(N)=\mathbf{a}_{D}^{\varepsilon}(N) a_{f}(N)$ for $(N, D)=1$. Note that $f^{\varepsilon *}$ is characterized as the unique element of $S_{2 k+1}\left(\Gamma_{0}(D), \chi\right)$ with this property.

Corollary 15.6. The form $f^{\varepsilon *}(\tau)=0$ if and only if $f(\tau)$ comes from a Hecke character of $\mathbb{Q}\left(\sqrt{\chi_{Q}(-1) D_{Q}}\right)$, for some $Q \subset Q_{D}, \varepsilon(Q) \chi_{Q}(-1)^{n}=-1$. In particular, $f^{*}=0$ if and only if $n$ is odd and $f(\tau)$ comes from a Hecke character $\mathbb{Q}\left(\sqrt{-D_{Q}}\right)$ for some $Q \subset Q_{D}, \chi_{Q}(-1)=-1$.

Proof. If $f(\tau)$ comes from a Hecke character of $\mathbb{Q}\left(\sqrt{\chi_{Q}(-1) D_{Q}}\right)$ with $\varepsilon(Q) \chi_{Q}(-1)^{n}=-1$, then obviously $f^{\varepsilon *}=0$, since $f^{\varepsilon *}=\left(f_{Q}\right)^{\varepsilon *}=-f^{\varepsilon *}$. Conversely, assume that $f^{\varepsilon *}=\sum_{Q \subset Q_{D}} \varepsilon(Q) \chi_{Q}(-1)^{n}$ $f_{Q}=0$. As the primitive forms are linearly independent, there exists a subset $Q \subset Q_{D}$ such that $f_{Q}=f$ and $\varepsilon(Q) \chi_{Q}(-1)^{n}=-1$. It follows that $f$ comes from a Hecke character of $\mathbb{Q}\left(\sqrt{\chi_{Q}(-1) D_{Q}}\right)$ by Labesse and Langlands [LL79].

Definition 15.7. For each primitive form $f \in S_{2 k+1}\left(\Gamma_{0}(D), \chi\right)$, put

$$
\eta_{n}^{\varepsilon}(f)=\sum_{\substack{Q \subset Q_{D} \\ f_{Q}=f}} \varepsilon(Q) \chi_{Q}(-1)^{n} .
$$




\section{LIFTING OF HERMITIAN MODULAR FORMS}

Lemma 15.8. If $k>0$, then $0 \leqslant \eta_{n}^{\varepsilon}(f) \leqslant 2$. When $k=0$, we have $0 \leqslant \eta_{n}^{\varepsilon}(f) \leqslant 4$.

Proof. That $\eta_{n}^{\varepsilon}(f) \leqslant 4$ follows from Labesse and Langlands [LL79]. To prove the first part, it is enough to prove that, if $k>0$, then there is no non-empty $Q \subset Q_{D}$ such that $f=f_{Q}, \chi_{Q}(-1)=1$. Assume that $f=f_{Q}, Q \neq \emptyset, \chi_{Q}(-1)=1$. Let $K_{Q}$ be the quadratic field corresponding to $\chi_{Q}$. Since $\chi_{Q}(-1)=1$, the quadratic field $K_{Q}$ is real. Then $f(\tau)$ comes from a Hecke character of $K_{Q}$. Comparing the gamma factor, it is impossible if $k>0$.

Lemma 15.9. We have $f^{\varepsilon *}=0$ if and only if $\eta_{n}^{\varepsilon}(f)=0$.

Proof. This lemma follows from Corollary 15.6.

Recall that the Petersson inner product of cusp forms $f_{1}, f_{2} \in S_{l}\left(\Gamma^{\prime}\right)$ for a congruence subgroup $\Gamma^{\prime} \subset \mathrm{SL}_{2}(\mathbb{Z})$ is given by

$$
\left\langle f_{1}, f_{2}\right\rangle=\left[\mathrm{SL}_{2}(\mathbb{Z}): \Gamma^{\prime} \cdot\{ \pm 1\}\right]^{-1} \int_{\Gamma^{\prime} \backslash \mathfrak{H}_{1}} f_{1}(\tau) \overline{f_{2}(\tau)} y^{l-2} d x d y
$$

The complete adjoint $L$-function $\Lambda(s, f, \mathrm{Ad})$ is defined by

$$
\begin{aligned}
\Lambda(s, f, \mathrm{Ad}) & =\Gamma_{\mathbb{R}}(s+1) \Gamma_{\mathbb{C}}(s+2 k) L(s, f, \mathrm{Ad}), \\
\Gamma_{\mathbb{R}}(s) & =\pi^{-s / 2} \Gamma(s / 2), \quad \Gamma_{\mathbb{C}}(s)=2(2 \pi)^{-s} \Gamma(s), \\
L(s, f, \mathrm{Ad}) & =\prod_{p \nmid D}\left[\left(1-\alpha_{p} \beta_{p}^{-1} p^{-s}\right)\left(1-p^{-s}\right)\left(1-\alpha_{p}^{-1} \beta_{p} p^{-s}\right)\right]^{-1} \prod_{q \mid D}\left(1-q^{-s}\right)^{-1} .
\end{aligned}
$$

The following lemma is well known (cf. e.g. Hida [Hid00, Theorem 5.15] for $k>0$ ).

Lemma 15.10. Let $f \in S_{2 k+1}\left(\Gamma_{0}(D), \chi\right)$ be a primitive form. Then we have

$$
\langle f, f\rangle=2^{-2 k-1} \Lambda(1, f, \text { Ad }) \prod_{q \mid D}\left(1+q^{-1}\right)^{-1},
$$

In particular, we have $\langle f, f\rangle=\left\langle f_{Q}, f_{Q}\right\rangle$ for any $Q \subset Q_{D}$.

Lemma 15.11. Put $t=\sharp Q_{D}$. Then we have

$$
\left\langle f^{*}, f^{*}\right\rangle=2^{\mathrm{t}} \eta_{n}(f)\langle f, f\rangle .
$$

Proof. We may assume that $f^{*} \neq 0$. Let $\left\{Q_{1}, Q_{2}, \ldots, Q_{l}\right\}$ be a maximal subset such that $\left\{f_{Q_{1}}\right.$, $\left.f_{Q_{2}}, \ldots, f_{Q_{l}}\right\}$ are linearly independent. Then $\eta_{n}(f) l=2^{\mathrm{t}}$ and $f^{*}=\eta_{n}(f) \sum_{i=1}^{l} f_{Q_{i}}$. Note that

$$
\left\langle f_{Q_{i}}, f_{Q_{j}}\right\rangle=0 \text { for } 1 \leqslant i, j \leqslant l, i \neq j,
$$

since $f_{Q_{1}}$ and $f_{Q_{2}}$ are different primitive forms. Therefore we have $\left\langle f^{*}, f^{*}\right\rangle=l \eta_{n}(f)^{2}\langle f, f\rangle=$ $2^{\mathrm{t}} \eta_{n}(f)\langle f, f\rangle$.

Proposition 15.12. The form $f^{*}(\tau)$ is identically zero if and only if $n$ is odd and $f(\tau)$ comes from a Hecke character of some imaginary quadratic field.

Proof. It is enough to prove that $f$ comes from a Hecke character of some quadratic field $K^{\prime}$, then $K^{\prime}=\mathbb{Q}\left(\sqrt{\chi_{Q}(-1) D_{Q}}\right)$ for some $Q \subset Q_{D}$. Let $\rho=\bigotimes_{v} \rho_{v}$ be the Hecke character of $\mathbb{A}^{\times} / \mathbb{Q}^{\times}$ corresponding to $K^{\prime} / \mathbb{Q}$. Then $\tilde{I}\left(\mathbf{1} \otimes \underline{\chi}_{p}, s_{0, p}\right)$ is isomorphic to $\tilde{I}\left(\rho_{p} \otimes \rho_{p} \underline{\chi}_{p}, s_{0, p}\right)$ for each prime $p$. Comparing the conductor, one can shows that either $\rho_{p}$ or $\rho_{p} \underline{\chi}_{p}^{-1}$ is unramified. It follows that $K^{\prime} K / K$ is unramified, and so $K^{\prime}=\mathbb{Q}\left(\sqrt{\chi_{Q}(-1) D_{Q}}\right)$ for some $Q \subset Q_{D}$ by genus theory.

Lemma 15.13. Let $f_{0}(\tau)$ be an element of $S_{2 k+1}\left(\Gamma_{0}(D), \chi\right)$. Assume that the $N$ th Fourier coefficient $a_{f_{0}}(N)$ is zero whenever $(N, D)=1$. Then $f_{0}=0$. 


\section{T. IKEDA}

Proof. This is a special case of Miyake [Miy89, Theorem 4.6.8].

Lemma 15.14. Let $N$ be a rational integer. Then there exists an integer $y \in \mathfrak{c}^{-1} \mathcal{O}^{\sharp}$ such that $C D y \bar{y} \equiv(-1)^{n} N \bmod D$ if and only if $\mathbf{a}_{D}^{\varepsilon}(N) \neq 0$.

Proof. As remarked in Krieg [Kri91, p. 670], we have

$$
\mathbf{a}_{D}(N)=\sharp\left\{u \in \mathcal{O}^{\sharp} / \mathcal{O} \mid D u \bar{u} \equiv(-1)^{n} N \bmod D\right\} .
$$

Choose $\alpha \in \mathfrak{c}$ such that $(\alpha, D)=1$. Put $\mathfrak{c}^{\prime}=(\alpha) \mathfrak{c}^{-1}, C^{\prime}=\mathrm{N}\left(\mathfrak{c}^{\prime}\right)$. Then $\mathfrak{c}$ and $\mathfrak{c}^{\prime}$ are integral ideals that belong to the same genus. Note that $\chi_{Q}(\mathrm{~N}(\alpha))=\chi_{Q}(C) \chi_{Q}\left(C^{\prime}\right)=1$ for any $Q \subset Q_{D}$. The map $y \mapsto u=\alpha y$ induces an isomorphism $\mathfrak{c}^{-1} \mathcal{O}^{\sharp} / \mathfrak{c}^{-1} \simeq \mathcal{O}^{\sharp} / \mathcal{O}$. Then we have

$$
\begin{aligned}
\mathbf{a}_{D}^{\varepsilon}(N)=\mathbf{a}_{D}\left(C^{\prime} N\right) & =\sharp\left\{u \in \mathcal{O}^{\sharp} / \mathcal{O} \mid D u \bar{u} \equiv(-1)^{n} C^{\prime} N\right\} \\
& =\sharp\left\{y \in \mathfrak{c}^{-1} \mathcal{O}^{\sharp} / \mathfrak{c}^{-1} \mid \mathrm{N}(\alpha) D y \bar{y} \equiv(-1)^{n} C^{\prime} N\right\} \\
& =\sharp\left\{y \in \mathfrak{c}^{-1} \mathcal{O}^{\sharp} / \mathfrak{c}^{-1} \mid C D y \bar{y} \equiv(-1)^{n} N\right\} .
\end{aligned}
$$

Hence the lemma is proved.

Lemma 15.15. Let $N$ be a positive integer. Then there exists an element $H \in \Lambda_{2 n}^{\mathfrak{c}}(\mathcal{O})^{+}$such that $C|\gamma(H)|=N$ if and only if $\mathbf{a}_{D}^{\varepsilon}(N) \neq 0$.

Proof. Assume that $\mathbf{a}_{D}^{\varepsilon}(N) \neq 0$. Then, by Lemmas 13.9 and 15.14 , there exists an element $H \in$ $\Lambda_{2 n}^{\mathfrak{c}}(\mathcal{O})^{+}$such that $C|\gamma(H)|=N$. Conversely, assume that $N=C|\gamma(H)|$ for $H \in \Lambda_{2 n}^{\mathfrak{c}}(\mathcal{O})^{+}$. It is enough to prove that $\underline{\chi}_{q}(N)=\underline{\chi}_{q}\left((-1)^{n} C\right)$ for any $q \mid D, q \nmid N$. Since $\gamma(H)=(-1)^{n} N C^{-1} \in \mathbb{Z}_{q}^{\times}$, we have $\tilde{F}_{q}(H, X)=1$. Then $\underline{\chi}_{q}(\gamma(H))=1$ by Lemma 2.2. Hence the lemma is proved.

DeFinition 15.16. Let $S_{2 k+1}^{\varepsilon *}\left(\Gamma_{0}(D), \chi\right)$ be the space of cusp forms

$$
f_{0}(\tau)=\sum_{N>0} a_{f_{0}}(N) q^{N} \in S_{2 k+1}\left(\Gamma_{0}(D), \chi\right)
$$

whose $N$ th Fourier coefficient is zero whenever $\mathbf{a}_{D}^{\varepsilon}(N)=0$. If $\varepsilon=1$, then $S_{2 k+1}^{\varepsilon *}\left(\Gamma_{0}(D), \chi\right)$ is simply denoted by $S_{2 k+1}^{*}\left(\Gamma_{0}(D), \chi\right)$.

Let $\left\{f_{i}\right\}_{i \in I}$ be the set of primitive forms in $S_{2 k+1}\left(\Gamma_{0}(D), \chi\right)$. By Corollary 15.5, $f_{i}^{\varepsilon *} \in S_{2 k+1}^{\varepsilon *}$ $\left(\Gamma_{0}(D), \chi\right)$. The following proposition is essentially Krieg [Kri91, p. 671, Proposition].

Proposition 15.17. The space $S_{2 k+1}^{\varepsilon *}\left(\Gamma_{0}(D), \chi\right)$ is spanned by $\left\{f_{i}^{\varepsilon *}\right\}_{i \in I}$.

Proof. Let $\left\{f_{i}\right\}_{i \in I}$ be the set of primitive forms of $S_{2 k+1}\left(\Gamma_{0}(D), \chi\right)$. Then it is well known that $\left\{f_{i}\right\}_{i \in I}$ is a basis of $S_{2 k+1}\left(\Gamma_{0}(D), \chi\right)$. Let $g$ be an element of $S_{2 k+1}^{\varepsilon *}\left(\Gamma_{0}(D), \chi\right)$. Then $g$ can be uniquely expressed as a linear combination $g=\sum_{i \in I} a_{i} \cdot f_{i}$. For $Q \subset Q_{D}$, set $g^{\prime}=\sum_{i \in I} a_{i} \cdot\left(f_{i}\right)_{Q}$. If $\left(D_{Q}, N\right)=1$, then the $N$ th Fourier coefficient of $g-\varepsilon(Q) \chi_{Q}(-1)^{n} g^{\prime}$ vanishes. By Lemma 15.13, we have $g=\varepsilon(Q) \chi_{Q}(-1)^{n} g^{\prime}$. Hence the lemma is proved.

Let $f \in S_{2 k+1}\left(\Gamma_{0}(D), \chi\right)$ be a primitive form. In terms of Satake parameters, $a_{f \varepsilon *}(N)$ can be expressed as follows. Put

$$
\Psi_{p}^{\varepsilon}(N ; X)= \begin{cases}\frac{X^{e_{p, N}+1}-\left(\xi_{p} X^{-1}\right)^{e_{p, N}+1}}{X-\xi_{p} X^{-1}} & p \notin Q_{D}, \\ X^{e_{p, N}}+\underline{\chi}_{p}\left((-1)^{n} C N\right) X^{-e_{p, N}} & p \in Q_{D},\end{cases}
$$

where $e_{p, N}=\operatorname{ord}_{p} N$ and $\xi_{p}=\chi(p)$. Put

$$
\Psi^{\varepsilon}(N ; \mathbb{X})=\prod_{p \mid D N} \Psi_{p}^{\varepsilon}\left(N ; X_{p}\right) \in \mathcal{R}
$$




\section{LIFTING OF HERMITIAN MODULAR FORMS}

Then we have $a_{f^{\varepsilon *}}(N)=N^{k} \Psi^{\varepsilon}\left(N ;\left\{\alpha_{p}\right\}\right)$ by Corollary 15.5. Note that $\Psi^{\varepsilon}(N ; \mathbb{X})=0$ if and only if $\mathbf{a}_{D}^{\varepsilon}(N)=0$.

Fix $H \in \Lambda_{2 n}^{\mathfrak{c}}(\mathcal{O})^{+}$. Then $\tilde{F}_{p}\left(\overline{\mathbf{t}}_{p} H \mathbf{t}_{p} ; X\right)$ belongs to the $\mathbb{Q}$-vector space

$$
\mathcal{V}=\left\{\Phi \in X^{-\operatorname{ord}_{p} C \gamma(H)} \mathbb{Q}\left[X^{2}\right] \mid \Phi\left(X^{-1}\right)=\underline{\chi}_{p}(C \gamma(H)) \Phi(X)\right\} .
$$

We put

$$
\mathcal{B}_{p}(H)=\left\{p^{r} \mid 0 \leqslant 2 r \leqslant \operatorname{ord}_{p}(C \gamma(H)), \mathbf{a}_{D}^{\varepsilon}\left(\frac{C|\gamma(H)|}{p^{2 r}}\right) \neq 0\right\}
$$

Since

$$
\left\{\Psi_{p}^{\varepsilon}\left(\frac{C|\gamma(H)|}{p^{2 r}} ; X\right) \mid p^{r} \in \mathcal{B}_{p}(H)\right\}
$$

is a basis of $\mathcal{V}$,

$$
F_{p}\left(\overline{\mathbf{t}}_{p} H \mathbf{t}_{p} ; X\right)=\sum_{p^{r} \in \mathcal{B}_{p}(H)} \phi_{p}\left(p^{r}, H\right) \Psi_{p}^{\varepsilon}\left(\frac{C|\gamma(H)|}{p^{2 r}} ; X\right)
$$

for some $\phi_{p}\left(p^{r}, H\right) \in \mathbb{Q}$. Note that

$$
\phi_{p}(1, H)= \begin{cases}\frac{1}{2} & p \in Q_{D}, p \nmid C \gamma(H), \\ 1 & \text { otherwise. }\end{cases}
$$

The set $\mathcal{B}(H)=\prod_{p} \mathcal{B}_{p}(H)$ can be identified with the set of positive integers $a$ such that $a^{2} \mid C \gamma(H)$ and $\mathbf{a}_{D}^{\varepsilon}\left(C|\gamma(H)| / a^{2}\right) \neq 0$. For each $a=\prod_{p \mid a} p^{r_{p}} \in \mathcal{B}(H)$, we put $\phi(a, H)=\prod_{p} \phi_{p}\left(p^{r_{p}}, H\right)$. Then we have

$$
\begin{aligned}
\prod_{p} \tilde{F}_{p}\left(\overline{\mathbf{t}}_{p} H \mathbf{t}_{p} ; X_{p}\right) & =\prod_{p}\left[\sum_{p^{r} \in \mathcal{B}_{p}(H)} \phi_{p}\left(p^{r}, H\right) \Psi_{p}^{\varepsilon}\left(\frac{C|\gamma(H)|}{p^{2 r}} ; X_{p}\right)\right] \\
& =\sum_{a \in \mathcal{B}(H)} \phi(a, H) \Psi^{\varepsilon}\left(\frac{C|\gamma(H)|}{a^{2}} ; \mathbb{X}\right) .
\end{aligned}
$$

Here we have used the fact that $\Psi_{p}^{\varepsilon}\left(a^{2} N ; X\right)=\Psi_{p}^{\varepsilon}(N ; X)$ for $p \nmid a$. Note that $1 \in \mathcal{B}(H)$ by Lemma 15.15. One can easily see that $\phi(1, H)=\mathbf{a}_{D}^{\varepsilon}(C|\gamma(H)|)^{-1} \neq 0$.

For each $f_{0}(\tau)=\sum_{N>0} a_{f_{0}}(N) q^{N} \in S_{2 k+1}^{\varepsilon *}\left(\Gamma_{0}(D), \chi\right)$, we put

$$
\iota\left(f_{0}\right)(Z)=\sum_{H \in \Lambda_{2 n}^{c}(\mathcal{O})+} \sum_{a \in \mathcal{B}(H)} a^{2 k} \phi(a, H) a_{f_{0}}\left(\frac{C|\gamma(H)|}{a^{2}}\right) \mathbf{e}(H Z) .
$$

If $f \in S_{2 k+1}\left(\Gamma_{0}(D), \chi\right)$ is a primitive form, then

$$
\iota\left(f^{\varepsilon *}\right)=C^{k+n} \operatorname{Lift}_{\mathfrak{c}}^{(2 n)}(f) \in S_{2 k+2 n}\left(\Gamma_{K}^{(2 n)}[\mathfrak{c}], \operatorname{det}^{-k-n}\right) .
$$

Since $\left\{f_{i}^{\varepsilon *}\right\}_{i \in I}$ spans $S_{2 k+1}^{\varepsilon *}\left(\Gamma_{0}(D), \chi\right)$, the image of $\iota$ is contained in $S_{2 k+2 n}\left(\Gamma_{K}^{(2 n)}[\mathfrak{c}], \operatorname{det}^{-k-n}\right)$.

Theorem 15.18. There exists an injective linear map

$$
\iota: S_{2 k+1}^{\varepsilon *}\left(\Gamma_{0}(D), \chi\right) \rightarrow S_{2 k+2 n}\left(\Gamma_{K}^{(2 n)}[\mathfrak{c}], \operatorname{det}^{-k-n}\right)
$$

satisfying the following properties:

(1) for each $f_{0} \in S_{2 k+1}^{\varepsilon *}\left(\Gamma_{0}(D), \chi\right)$, we have

$$
\iota\left(f_{0}\right)(Z)=\sum_{H \in \Lambda_{2 n}^{\mathfrak{c}}(\mathcal{O})+} \sum_{a \in \mathcal{B}(H)} a^{2 k} \phi(a, H) a_{f_{0}}\left(\frac{C|\gamma(H)|}{a^{2}}\right) \mathbf{e}(H Z),
$$




\section{T. IKEDA}

where

$$
\mathcal{B}(H)=\left\{a \in \mathbb{Z}\left|a>0, a^{2}\right| C \gamma(H), \mathbf{a}_{D}\left(\frac{C|\gamma(H)|}{a^{2}}\right) \neq 0\right\}
$$

(2) if $f$ is a primitive form in $S_{2 k+1}\left(\Gamma_{0}(D)\right.$, $\left.\operatorname{det}^{-k-n}\right)$, then $\iota\left(f^{\varepsilon *}\right)=C^{k+n} \operatorname{Lift}_{\mathfrak{c}}^{(2 n)}(f)$.

Proof. We need to prove the injectivity of $\iota$. Assume that $\iota\left(f_{0}\right)=0$ for $f_{0} \in S_{2 k+1}^{\varepsilon *}\left(\Gamma_{0}(D), \chi\right)$. We have to show that $a_{f_{0}}(N)=0$ for $\mathbf{a}_{D}^{\varepsilon}(N) \neq 0$. By Lemma 15.15, there exists an element $H_{N} \in \Lambda_{m}^{\mathfrak{c}}(\mathcal{O})^{+}$such that $C\left|\gamma\left(H_{N}\right)\right|=N$. As in Case O, the $H_{N}$ th Fourier coefficient of $\iota\left(f_{0}\right)$ is equal to $\mathbf{a}_{D}^{\varepsilon}(N)^{-1} a_{f_{0}}(N)+$ (lower terms). By induction, we have $a_{f_{0}}(N)=0$.

Corollary 15.19. Let $f(\tau) \in S_{2 k+1}\left(\Gamma_{0}(D), \chi\right)$ be a primitive form. Then $\operatorname{Lift}_{\mathfrak{c}}(f)=0$ if and only if $f$ comes from a Hecke character of a field $\mathbb{Q}\left(\sqrt{\chi_{Q}(-1) D_{Q}}\right)$ such that $\varepsilon(Q) \chi_{Q}(-1)^{n}=-1$.

Corollary 15.20. Let $f(\tau) \in S_{2 k+1}\left(\Gamma_{0}(D), \chi\right)$ be a primitive form. Then the following conditions are equivalent:

(1) $\operatorname{Lift}^{(2 n)}(f)=0$;

(2) $f^{*}=0$;

(3) $n$ is odd and $f(\tau)$ comes from a Hecke character $\mathbb{Q}\left(\sqrt{-D_{Q}}\right)$ for some $Q \subset Q_{D}, \chi_{Q}(-1)=-1$;

(4) $n$ is odd and $f$ comes from a Hecke character of some imaginary quadratic field.

Corollary 15.21. Let $f(\tau) \in S_{2 k+1}\left(\Gamma_{0}(D), \chi\right)$ be a primitive form. Then Lift ${ }^{(2 n)}(f) \in \mathcal{S}_{2 k+2 n}$ $\left(\mathcal{G}(\mathbb{Q}) \backslash \mathcal{G}(\mathbb{A}), \operatorname{det}^{-k-n}\right)$ is identically zero if and only if $n$ is odd and $f$ comes from a Hecke character of $K$.

Proof. Note that Lift ${ }^{(2 n)}(f)=0$ if and only if $f^{\varepsilon *}=0$ for any $\varepsilon$. Assume that $f^{\varepsilon *}=0$ for any $\varepsilon$. Then $n$ is odd, since otherwise $f^{*} \neq 0$. If $n$ is odd, we have

$$
f-f_{Q_{D}}=2^{1-t} \sum_{\varepsilon} f^{\varepsilon *}=0
$$

where $t=\sharp Q_{D}$. Conversely, if $n$ is odd and $f=f_{Q_{D}}$, then $f^{\varepsilon *}=0$ for any $\varepsilon$. Hence the corollary is proved.

\section{An example: the case $m=2$}

The case $m=2$ was first considered by Kojima $[$ Koj82] for $K=\mathbb{Q}(\sqrt{-1})$ and later by Gritsenko [Gri90]. Krieg [Kri91] and Sugano [Sug85] investigated the Maass spaces for arbitrary imaginary quadratic field. In this case, $\operatorname{Lift}^{(2)}(f)$ is called the Maass lift of $f$. Recently, Klosin [Klo07b] defined the Maass space for $\mathrm{U}(2,2)$ in the adelic setting, and constructed the extension of $\operatorname{Lift}^{(2)}(f)$ under the assumption that the class number $h_{K}$ is odd. As we described in $\S 15$, there is an injective linear map $S_{2 k+1}^{*}\left(\Gamma_{0}(D), \chi\right)^{h_{K}} \rightarrow \mathcal{S}_{2 k+2}\left(\mathcal{G}(\mathbb{Q}) \backslash \mathcal{G}(\mathbb{A})\right.$, det $\left.{ }^{-k-1}\right)$ in this case.

We do not give a detailed proof for the results in this section, as most of the results are not new, and are contained in the references above (at least when $\mathfrak{c}=\mathcal{O}$ ).

Let $\mathfrak{c}$ be an integral ideal of $K$ such that $C=\mathrm{N}(\mathfrak{c})$ is prime to $D$.

DeFinition 16.1. The function

$$
F(Z)=\sum_{H \in \Lambda_{2}^{\mathfrak{c}}(\mathcal{O})} A_{F}(H) \mathbf{e}(H Z) \in M_{2 k+2}\left(\Gamma_{K}^{(2)}[\mathfrak{c}], \operatorname{det}^{-k-1}\right)
$$

satisfies the Maass relation if and only if there is a function

$$
\alpha_{F}^{*}: \mathbb{Z}_{\geqslant 0} \rightarrow \mathbb{C}
$$




\section{LIFTING OF HERMITIAN MODULAR FORMS}

such that

$$
A_{F}(H)=\sum_{d \mid \varepsilon(H)} d^{2 k+1} \alpha_{F}^{*}\left(\frac{C|\gamma(H)|}{d^{2}}\right) \text { for } H \neq 0
$$

Here

$$
\varepsilon(H)=\max \left\{q \in \mathbb{Z}_{>0} \mid q^{-1} H \in \Lambda_{2}^{\mathfrak{c}}(\mathcal{O})\right\} .
$$

Note that the values $\alpha_{F}^{*}(N)$ for $\mathbf{a}_{D}^{\varepsilon}(N)=0$ play no role. We denote the space of elements of $M_{2 k+2}\left(\Gamma_{K}^{(2)}, \operatorname{det}^{-k-1}\right)$ satisfying the Maass relation by $M_{2 k+2}^{\text {Maass }}\left(\Gamma_{K}^{(2)}\right.$, $\left.\operatorname{det}^{-k-1}\right)$. We set $S_{2 k+2}^{\text {Maass }}$ $\left(\Gamma_{K}^{(2)}, \operatorname{det}^{-k-1}\right)=M_{2 k+2}^{\text {Maass }}\left(\Gamma_{K}^{(2)}, \operatorname{det}^{-k-1}\right) \cap S_{2 k+2}\left(\Gamma_{K}^{(2)}, \operatorname{det}^{-k-1}\right)$.

It is known that the normalized hermitian Eisenstein series

$$
F=\mathcal{E}_{2 k+2}^{(2)}(Z)=\frac{B_{2 k+2} B_{2 k+1, \chi}}{8(k+1)(2 k+1)} E_{2 k+2}^{(2)}(Z)
$$

satisfies the Maass relation for $\mathfrak{c}=\mathcal{O}$. The function $\alpha_{F}^{*}$ is given by

$$
\alpha_{F}^{*}(N)= \begin{cases}0 & \mathbf{a}_{D}(N)=0, \\ -B_{2 k+1, \chi} /(4 k+2) & N=0, \\ \mathbf{a}_{D}(N)^{-1} \sum_{d \mid N} \sum_{Q \subset Q_{D}} \chi_{Q}(-N / d) \chi_{Q}^{\prime}(d) d^{2 k} & N>0, \mathbf{a}_{D}(N) \neq 0\end{cases}
$$

(cf. Krieg [Kri91, p. 679]; Krieg [Kri91] assumed $w_{K} \mid(2 k+2)$, but the modification is easy). Using these results, one can calculate the Laurent polynomial $\tilde{F}_{p}(H ; X)$ as follows:

$$
\tilde{F}_{p}(H ; X)= \begin{cases}\sum_{i=0}^{b} p^{i} \sum_{j=0}^{a-2 i} \underline{\chi}_{p}(p)^{j} X^{a-2 i-2 j} & \text { if } p \nmid D, \\ \sum_{i=0}^{b} p^{i}\left(X^{a-2 i}+\underline{\chi}_{p}(\gamma(H)) X^{-a+2 i}\right) & \text { if } p \mid D, 2 b<a, \\ p^{b}+\sum_{i=0}^{b-1} p^{i}\left(X^{a-2 i}+X^{-a+2 i}\right) & \text { if } p \mid D, 2 b=a .\end{cases}
$$

Here $a=\operatorname{ord}_{p} \gamma(H)$, and $b=\operatorname{ord}_{p} \varepsilon(H)$. When the class number of $K$ is 1 , this has already been essentially calculated by Nagaoka [Nag92, Theorem 1.3.1]. Using this formula, we have

$$
\prod_{p} \tilde{F}_{p}\left(\overline{\mathbf{t}}_{p} H \mathbf{t}_{p} ; X_{p}\right)=\sum_{d \in \varepsilon(H)} d \mathbf{a}_{D}^{\varepsilon}\left(\frac{C|\gamma(H)|}{d^{2}}\right)^{-1} \Psi^{\varepsilon}\left(\frac{C|\gamma(H)|}{d^{2}} ; \mathbb{X}\right)
$$

for $H \in \Lambda_{2}^{\mathfrak{c}}(\mathcal{O})^{+}$. It follows that the map

$$
\iota: S_{2 k+1}^{\varepsilon *}\left(\Gamma_{0}(D), \chi\right) \rightarrow S_{2 k+2}\left(\Gamma_{K}^{(2)}[\mathfrak{c}], \operatorname{det}^{-k-1}\right)
$$

is given by

$$
\iota(f)(Z)=\sum_{H \in \Lambda_{2}(\mathcal{O})+} \sum_{d \in \varepsilon(H)} d^{2 k+1} \mathbf{a}_{D}^{\varepsilon}\left(\frac{C|\gamma(H)|}{a^{2}}\right)^{-1} a_{f}\left(\frac{C|\gamma(H)|}{a^{2}}\right) \mathbf{e}(H Z) .
$$

In particular, the image of $\iota$ is contained in $S_{2 k+2}^{\text {Maass }}\left(\Gamma_{K}^{(2)}[\mathfrak{c}], \operatorname{det}^{-k-1}\right)$.

For $F \in S_{2 k+2}\left(\Gamma_{K}^{(2)}[\mathfrak{c}], \operatorname{det}^{-k-1}\right)$, consider the first Fourier-Jacobi coefficient

$$
\phi_{1}\left(\tau, z_{1}, z_{2}\right)=\sum_{\substack{l \in C^{-1} \mathbb{Z} \\
t \in \mathfrak{c}^{-1} \mathcal{O}^{\sharp}}} A_{F}\left(\left(\begin{array}{cc}
1 & t \\
\bar{t} & l
\end{array}\right)\right) \mathbf{e}\left(l \tau+\bar{t} z_{1}+t z_{2}\right) .
$$




\section{T. IKEDA}

Then there exist functions $f_{[u]}(\tau)\left(u \in \mathfrak{c}^{-1} \mathcal{O}^{\sharp} / \mathfrak{c}^{-1}\right)$ such that

$$
\phi_{1}\left(\tau, z_{1}, z_{2}\right)=\sum_{u \in \mathfrak{c}^{-1} \mathcal{O}^{\sharp} / \mathfrak{c}^{-1}} \theta_{[u]}\left(\tau, z_{1}, z_{2}\right) f_{[u]}(\tau / C),
$$

where

$$
\theta_{[u]}\left(\tau, z_{1}, z_{2}\right)=\sum_{a \in u+\mathfrak{c}^{-1}} \mathbf{e}\left(a \bar{a} \tau+\bar{a} z_{1}+a z_{2}\right)
$$

Then one can show that:

(1) $f_{[u]} \in S_{2 k+1}(\Gamma(D))$, where $\Gamma(D)$ is the principal congruence subgroup of $\mathrm{SL}_{2}(\mathbb{Z})$ modulo $D$;

(2) $f_{[u]}(\tau+1)=\mathbf{e}(-C u \bar{u}) f_{[u]}$;

(3) $\left.f_{[u]}\right|_{2 k+1}\left[\begin{array}{rr}0 & -1 \\ 1 & 0\end{array}\right]=-(1 / \sqrt{-D}) \sum_{v \in \mathfrak{c}^{-1} \mathcal{O}^{\sharp} / \mathfrak{c}^{-1}} \mathbf{e}(C(u \bar{v}+v \bar{u})) f_{[v]}$;

(4) $f_{[0]} \in S_{2 k+1}\left(\Gamma_{0}(D), \chi\right)$.

If $\mathfrak{c}=\mathcal{O}$, this is proved in Krieg [Kri91, p. 669]. One can easily treat the general case by using the theta transformation formula for a hermitian theta function (see Shintani [Shi75], Shimura [Shi97, A7]). Set

$$
\Omega(F)(\tau)=\sum_{u \in \mathfrak{c}^{-1} \mathcal{O}^{\sharp} / \mathfrak{c}^{-1}} f_{[u]}(D \tau) .
$$

Then we have $\Omega(F) \in S_{2 k+1}^{\varepsilon *}\left(\Gamma_{0}(D), \chi\right)$. If $F \in S_{2 k+2}^{\text {Maass }}\left(\Gamma_{K}^{(2)}[\mathfrak{c}], \operatorname{det}^{-k-1}\right)$, then we have

$$
\begin{aligned}
f_{[u]}(\tau) & =\sum_{N \equiv-C D u \bar{u} \bmod D} \alpha_{F}^{*}(N) \mathbf{e}(N \tau / D), \\
\Omega(F)(\tau) & =\sum_{N>0} \mathbf{a}_{D}^{\varepsilon}(N) \alpha_{F}^{*}(N) \mathbf{e}(N \tau) .
\end{aligned}
$$

It follows that $\iota$ gives the isomorphism between $S_{2 k+1}^{\varepsilon *}\left(\Gamma_{0}(D), \chi\right)$ and $S_{2 k+2}^{\text {Maass }}\left(\Gamma_{K}^{(2)}[\mathfrak{c}], \operatorname{det}^{-k-1}\right)$.

\section{Petersson norms of $\operatorname{Lift}^{(m)}(f)$}

We recall the definition of the Petersson inner product for hermitian modular forms. For $F_{1}, F_{2} \in$ $S_{l}\left(\Gamma_{K}^{(m)}, \sigma\right)$, the Pertersson inner product $\left\langle F_{1}, F_{2}\right\rangle$ is defined by

$$
\left\langle F_{1}, F_{2}\right\rangle=\int_{\Gamma_{K}^{(m)} \backslash \mathcal{H}_{m}} F_{1}(Z) \overline{F_{2}(Z)}(\operatorname{det} Y)^{l-2 m} d X d Y,
$$

where $X=\left(Z+{ }^{\mathrm{t}} \bar{Z}\right) / 2$, and $Y=\left(Z-{ }^{\mathrm{t}} \bar{Z}\right) /(2 \sqrt{-1})$. The measure $d X$ on the space of hermitian matrices is defined by $d X=\prod_{i \leqslant j} d X_{i j}^{(r)} \prod_{i<j} d X_{i j}^{(i)}$, where $X=X^{(r)}+\sqrt{-1} X^{(i)}$, and $X_{i j}^{(r)}, X_{i j}^{(i)} \in \mathbb{R}$.

In this section, we investigate the Petersson norm of the lifts of $f$. For simplicity, we only consider the case $\mathfrak{c}=\mathcal{O}$.

Let $f \in S_{2 k+1}\left(\Gamma_{0}(D), \chi\right)$ be a primitive form. Put $F=\operatorname{Lift}^{(2)}(f) \in S_{2 k+2}\left(\Gamma_{K}^{(2)}, \operatorname{det}^{-k-1}\right)$. As we have seen in $\S 16$, the first Fourier-Jacobi coefficient $\phi_{1}$ of $F$ has the decomposition

$$
\begin{aligned}
\phi_{1}\left(\tau, z_{1}, z_{2}\right) & =\sum_{u \in \mathcal{O}^{\sharp} / \mathcal{O}} f_{[u]}(\tau) \theta_{[u]}\left(\tau, z_{1}, z_{2}\right), \\
\theta_{[u]}\left(\tau, z_{1}, z_{2}\right) & =\sum_{a \equiv u \bmod \mathcal{O}} \mathbf{e}\left(a \bar{a} \tau+\bar{a} z_{1}+a z_{2}\right),
\end{aligned}
$$




\section{LIFTING OF HERMITIAN MODULAR FORMS}

where

$$
f_{[u]}(\tau)=\sum_{N \equiv-D u \bar{u} \bmod D} \alpha_{F}^{*}(N) \mathbf{e}(N \tau / D)
$$

for each $u \in \mathcal{O}^{\sharp} / \mathcal{O}$. Note that $f^{*}(\tau)=\sum_{u \in \mathcal{O}^{\sharp} / \mathcal{O}} f_{[u]}(D \tau)$.

The Petersson inner product $\left\langle\phi_{1}, \phi_{1}\right\rangle$ is defined by

$$
\int_{J \backslash\left(\mathfrak{h}_{1} \times \mathbb{C}^{2}\right)} \phi_{1}\left(\tau, z_{1}, z_{2}\right) \overline{\phi_{1}\left(\tau, z_{1}, z_{2}\right)} y^{l-4} e^{-\pi\left|z_{1}-\bar{z}_{2}\right|^{2} / y} d t_{1} d w_{1} d t_{2} d w_{2} d x d y .
$$

Here, $J=J_{2,1}(\mathcal{O}), \tau=x+\sqrt{-1} y, z_{1}=t_{1}+\sqrt{-1} w_{1}$, and $z_{2}=t_{2}+\sqrt{-1} w_{2}$.

Proposition 17.1. We have

$$
\left\langle\phi_{1}, \phi_{1}\right\rangle=\frac{\sqrt{D}}{4} \sum_{u \in \mathcal{O} \sharp / \mathcal{O}}\left\langle f_{[u]}, f_{[u]}\right\rangle .
$$

Proof (cf. [EZ85, Theorem 5.3]). Since the non-trivial element of the center of $\mathrm{SL}_{2}(\mathbb{Z})$ acts on $\mathbb{C}^{2} / L_{\tau}$ by $\left(z_{1}, z_{2}\right) \mapsto\left(-z_{1},-z_{2}\right)$, we have

$$
J \backslash\left(\mathfrak{h}_{1} \times \mathbb{C}^{2}\right)=\left\{\left(\tau, z_{1}, z_{2}\right) \mid \tau \in \mathrm{SL}_{2}(\mathbb{Z}) \backslash \mathfrak{h}_{1},\left(z_{1}, z_{2}\right) \in\left(\mathbb{C}^{2} / L_{\tau}\right) /\{ \pm 1\}\right\} .
$$

It follows that $\left\langle\phi_{1}, \phi_{1}\right\rangle$ is equal to

$$
\begin{aligned}
& \frac{1}{2}\left[\mathrm{SL}_{2}(\mathbb{Z}): \Gamma(D)\{ \pm 1\}\right]^{-1} \int_{\tau \in\left(\Gamma(D) \backslash \mathfrak{h}_{1}\right)} \sum_{u, v \in \mathcal{O}^{\sharp} / \mathcal{O}} f_{[u]}(\tau) \overline{f_{[v]}(\tau)} y^{2 k-2} \\
& \times \int_{\mathbb{C}^{2} / L_{\tau}} \theta_{[u]}\left(\tau, z_{1}, z_{2}\right) \overline{\theta_{[v]}\left(\tau, z_{1}, z_{2}\right)} e^{-\pi y\left|z_{1}-\bar{z}_{2}\right|^{2}} d t_{1} d w_{1} d t_{2} d w_{2} d x d y,
\end{aligned}
$$

where $L_{\tau}=\{(\lambda \tau+\mu, \bar{\lambda} \tau+\bar{\mu}) \mid \lambda, \mu \in \mathcal{O}\}$. It is easy to show that

$$
\int_{\mathbb{C}^{2} / L_{\tau}} \theta_{[u]}\left(\tau, z_{1}, z_{2}\right) \overline{\theta_{[v]}\left(\tau, z_{1}, z_{2}\right)} e^{-\pi\left|z_{1}-\bar{z}_{2}\right|^{2} / y} d t_{1} d w_{1} d t_{2} d w_{2}=\delta_{u v} \frac{\sqrt{D}}{2} y .
$$

Hence the proposition is proved.

Lemma 17.2. Set $N_{u}=-D u \bar{u} \in \mathbb{Z} / D \mathbb{Z}$ for each $u \in \mathcal{O}^{\sharp} / \mathcal{O}$. Then we have

$$
\left\langle f_{[u]}, f_{[u]}\right\rangle=\mathbf{a}_{D}\left(N_{u}\right)^{-1}\left\langle f_{[0]}, f_{[0]}\right\rangle .
$$

Proof. Let $\sum_{u \in \mathcal{O} \sharp / \mathcal{O}} \mathbb{C} f_{[u]}$ be the space generated by $\left\{f_{[u]} \mid u \in \mathcal{O}^{\sharp} / \mathcal{O}\right\}$. It is well known that the space $\sum_{u \in \mathcal{O}^{\sharp} / \mathcal{O}} \mathbb{C} f_{[u]}$ can be naturally identified with a subrepresentation of the (finite) Weil representation (cf. Shintani $\left[\right.$ Shi75]). Let $\mathbb{C}\left[\mathcal{O}^{\sharp} / \mathcal{O}\right]=\bigoplus_{q \mid D} \mathbb{C}\left[\mathcal{O}_{q}^{\sharp} / \mathcal{O}_{q}\right]$ be the space of functions on $\mathcal{O}^{\sharp} / \mathcal{O}=\bigoplus_{q \mid D} \mathcal{O}_{q}^{\sharp} / \mathcal{O}_{q}$, where $\mathcal{O}_{q}^{\sharp}=\mathcal{O}^{\sharp} \otimes \mathcal{O} \mathcal{O}_{q}$. Let $\varphi_{u} \in \mathbb{C}\left[\mathcal{O}^{\sharp} / \mathcal{O}\right]$ be the characteristic function of $u \in \mathcal{O}^{\sharp} / \mathcal{O}$. Recall that there exists a representation, called the (finite) Weil representation $\omega$ of $\mathrm{SL}_{2}(\mathbb{Z} / D \mathbb{Z})$ on $\mathbb{C}\left[\mathcal{O}^{\sharp} / \mathcal{O}\right]$, that is characterized by:

(i) $\omega\left(\begin{array}{ll}1 & 1 \\ 0 & 1\end{array}\right) \varphi_{u}=\mathbf{e}(-u \bar{u}) \varphi_{u}$;

(ii) $\omega\left(\begin{array}{cc}0 & -1 \\ 1 & 0\end{array}\right) \varphi_{u}=-(1 / \sqrt{-D}) \sum_{v \in \mathcal{O}^{\sharp} / \mathcal{O}} \mathbf{e}(u \bar{v}+v \bar{u}) \varphi_{v}$.

The Weil representation $\omega$ is a unitary representation with respect to the natural inner product on $\mathbb{C}\left[\mathcal{O}^{\sharp} / \mathcal{O}\right]=L^{2}\left(\mathcal{O}^{\sharp} / \mathcal{O}\right)$. For each prime $q \in Q_{D}$, let $\mathcal{U}_{q}$ be the kernel of the norm map $N_{K_{q} / Q_{q}}$ : $K_{q}^{\times} \rightarrow \mathbb{Q}_{q}^{\times}$. Note that the group $\mathcal{U}=\prod_{q \mid D} \mathcal{U}_{q}$ acts on $\mathbb{C}\left[\mathcal{O}^{\sharp} / \mathcal{O}\right]$. This action commutes with the action $\omega$ of $\mathrm{SL}_{2}(\mathbb{Z} / D \mathbb{Z})$. We denote by $\mathbb{C}\left[\mathcal{O}^{\sharp} / \mathcal{O}\right]^{\mathcal{U}}$ the space of $\mathcal{U}$-invariants in $\mathbb{C}\left[\mathcal{O}^{\sharp} / \mathcal{O}\right]$. This is an irreducible subrepresentation of $\omega$. Then the space $\sum_{u \in \mathcal{O}^{\sharp} / \mathcal{O}} \mathbb{C} f_{[u]}$ is isomorphic to $\mathbb{C}\left[\mathcal{O}^{\sharp} / \mathcal{O}\right]^{\mathcal{U}}$ as a 


\section{T. IKEDA}

representation of $\mathrm{SL}_{2}(\mathbb{Z} / D \mathbb{Z})$. Put

$$
\tilde{\varphi}_{u}=\mathbf{a}_{D}\left(N_{u}\right)^{-1} \sum_{\substack{v \in \mathcal{O}^{\sharp} / \mathcal{O} \\ N_{v}=N_{u}}} \varphi_{v} .
$$

Then $f_{[u]}$ corresponds to $\tilde{\varphi}_{u}$ under the isomorphism. Clearly we have $\mathbf{a}_{D}\left(N_{u}\right)\left\|\tilde{\varphi}_{u}\right\|^{2}=\left\|\tilde{\varphi}_{0}\right\|^{2}$, since $\mathbf{a}_{D}\left(N_{u}\right)=\#\left\{v \in \mathcal{O}^{\sharp} / \mathcal{O} \mid N_{v}=N_{u}\right\}$.

Proposition 17.3. We have

$$
\sum_{u \in \mathcal{O}^{\sharp} / \mathcal{O}}\left\langle f_{[u]}, f_{[u]}\right\rangle=D^{2 k+1}\left(\prod_{q \mid D} \frac{1}{2}\left(1+q^{-1}\right)\right)\left\langle f^{*}, f^{*}\right\rangle .
$$

Proof. By Lemma 17.2, we have

$$
\begin{aligned}
\sum_{u \in \mathcal{O}^{\sharp} / \mathcal{O}}\left\langle f_{[u]}, f_{[u]}\right\rangle & =\left(\sum_{u \in \mathcal{O}^{\sharp} / \mathcal{O}} \mathbf{a}_{D}\left(N_{u}\right)^{-1}\right)\left\langle f_{[0]}, f_{[0]}\right\rangle \\
& =\sharp\left\{N \bmod D \mid \mathbf{a}_{D}(N) \neq 0\right\} \cdot\left\langle f_{[0]}, f_{[0]}\right\rangle \\
& =D\left(\prod_{q \mid D} \frac{1}{2}\left(1+q^{-1}\right)\right)\left\langle f_{[0]}, f_{[0]}\right\rangle .
\end{aligned}
$$

Since

$$
\sum_{u \in \mathcal{O}^{\sharp} / \mathcal{O}} f_{[u]}=-\left.\sqrt{-D} f_{[0]}\right|_{2 k+1}\left[\begin{array}{rr}
0 & -1 \\
1 & 0
\end{array}\right],
$$

we have

$$
D\left\langle f_{[0]}, f_{[0]}\right\rangle=\left\langle\sum_{u \in \mathcal{O}^{\sharp} / \mathcal{O}} f_{[u]}, \sum_{u \in \mathcal{O}^{\sharp} / \mathcal{O}} f_{[u]}\right\rangle .
$$

Since $f^{*}(\tau)=\sum_{u \in \mathcal{O}^{\sharp} / \mathcal{O}} f_{[u]}(D \tau)$, the proposition follows.

By Lemmas 15.10 and 15.11, and Propositions 17.1 and 17.3, we have

$$
\left\langle\phi_{1}, \phi_{1}\right\rangle=2^{-2 k-3} D^{2 k+(3 / 2)} \eta_{1}(f) \Lambda(1, f, \mathrm{Ad}) .
$$

Sugano [Sug95, Corollary 8.3] has proved that

$$
\langle F, F\rangle=2^{-2 k-5} D^{3 / 2} \pi^{-2} \xi(2) \Lambda(2, f, \mathrm{Ad}, \chi)\left\langle\phi_{1}, \phi_{1}\right\rangle,
$$

where

$$
\begin{aligned}
\xi(s)= & \Gamma_{\mathbb{R}}(s) \zeta(s), \\
\Lambda(s, f, \mathrm{Ad}, \chi)= & \Gamma_{\mathbb{R}}(s) \Gamma_{\mathbb{C}}(s+2 k) L(s, f, \mathrm{Ad}, \chi), \\
L(s, f, \mathrm{Ad}, \chi)= & \prod_{p \nmid D}\left[\left(1-\alpha_{p}^{2} p^{-s}\right)\left(1-\chi(p) p^{-s}\right)\left(1-\beta_{p}^{2} p^{-s}\right)\right]^{-1} \\
& \times \prod_{q \mid D}\left[\left(1-\alpha_{q}^{2} q^{-s}\right)\left(1-\alpha_{q}^{-2} q^{-s}\right)\right]^{-1} .
\end{aligned}
$$

Note that Sugano has formulated his theorems in terms of orthogonal groups. In particular, the normalization of the inner products is different from our normalization. By combining our calculation and Sugano's result, we obtain the following proposition.

Proposition 17.4. Let $f \in S_{2 k+1}\left(\Gamma_{0}(D), \chi\right)$ be a primitive form, and put $F=\operatorname{Lift}^{(2)}(f)$. Then we have

$$
\langle F, F\rangle=\eta_{1}(f) 2^{-4 k-8} D^{2 k+3} \pi^{-2} \xi(2) \Lambda(1, f, \operatorname{Ad}) \Lambda(2, f, \operatorname{Ad}, \chi) .
$$




\section{LIFTING OF HERMITIAN MODULAR FORMS}

Proposition 17.4 can be proved by using the method of [RS91]. As in Ikeda [Ike06], we can give a conjecture on the Petersson norm of $\operatorname{Lift}^{(m)}(f)$. We define the modified complete $L$-functions as follows. Put

$$
\tilde{\Lambda}\left(s, \chi^{i}\right)= \begin{cases}\Gamma_{\mathbb{C}}(s) \zeta(s) & \text { if } i \text { is even, } \\ \Gamma_{\mathbb{C}}(s) L(s, \chi) & \text { if } i \text { is odd. }\end{cases}
$$

For a normalized eigenform $f \in S_{2 k}\left(\mathrm{SL}_{2}(\mathbb{Z})\right)$, we put

$$
\begin{aligned}
\tilde{\Lambda}\left(s, f, \mathrm{Ad}, \chi^{i}\right) & =\Gamma_{\mathbb{C}}(s) \Gamma_{\mathbb{C}}(s+2 k-1) \times \begin{cases}L(s, f, \mathrm{Ad}) & \text { if } i \text { is even, } \\
L(s, f, \mathrm{Ad}, \chi) & \text { if } i \text { is odd, }\end{cases} \\
L(s, f, \mathrm{Ad}) & =\prod_{p}\left[\left(1-\alpha_{p}^{2} p^{-s}\right)\left(1-p^{-s}\right)\left(1-\beta_{p}^{2} p^{-s}\right)\right]^{-1}, \\
L(s, f, \mathrm{Ad}, \chi) & =\prod_{p}\left[\left(1-\alpha_{p}^{2} \chi(p) p^{-s}\right)\left(1-\chi(p) p^{-s}\right)\left(1-\beta_{p}^{2} \chi(p) p^{-s}\right)\right]^{-1} .
\end{aligned}
$$

Similarly, for a primitive form $f \in S_{2 k+1}\left(\Gamma_{0}(D), \chi\right)$, we put

$$
\tilde{\Lambda}\left(s, f, \mathrm{Ad}, \chi^{i}\right)=\Gamma_{\mathbb{C}}(s) \Gamma_{\mathbb{C}}(s+2 k) \times \begin{cases}L(s, f, \mathrm{Ad}) & \text { if } i \text { is even, } \\ L(s, f, \mathrm{Ad}, \chi) & \text { if } i \text { is odd } .\end{cases}
$$

Conjecture 17.5. Let $f \in S_{2 k}\left(\mathrm{SL}_{2}(\mathbb{Z})\right)$ be a normalized Hecke eigenform. Then the Petersson norm of $F=\operatorname{Lift}^{(2 n+1)}(f)$ is given by

$$
\langle F, F\rangle=2^{\alpha} D^{\beta} \tilde{\Lambda}(1, f, \mathrm{Ad}) \prod_{i=2}^{2 n+1} \tilde{\Lambda}\left(i, \chi^{i}\right) \tilde{\Lambda}\left(i, f, \mathrm{Ad}, \chi^{i-1}\right)
$$

for some integers $\alpha$ and $\beta$ depending only on $n$ and $k$.

Conjecture 17.6. Let $f \in S_{2 k+1}\left(\Gamma_{0}(D), \chi\right)$ be a primitive form. Then the Petersson norm of $F=\operatorname{Lift}^{(2 n)}(f)$ is given by

$$
\langle F, F\rangle=\eta_{n}(f) 2^{\gamma} D^{\delta} \tilde{\Lambda}(1, f, \mathrm{Ad}) \prod_{i=2}^{2 n} \tilde{\Lambda}\left(i, \chi^{i}\right) \tilde{\Lambda}\left(i, f, \mathrm{Ad}, \chi^{i-1}\right)
$$

for some integers $\gamma$ and $\delta$ depending only on $n$ and $k$. For the definition of $\eta_{n}(f)$, see Definition 15.7.

\section{An interpretation in terms of automorphic representations}

Let $f$ be as in Theorem 5.1 or as in Theorem 5.2. Now we consider the L-function of Lift ${ }^{(m)}(f)$. Recall that the $L$-group of $\mathcal{G}=\mathrm{U}(m, m)$ is described as

$$
{ }^{L} \mathcal{G}=\mathrm{GL}_{2 m}(\mathbb{C}) \rtimes W_{\mathbb{Q}},
$$

where $W_{\mathbb{Q}}$ is the Weil group of $\mathbb{Q}$. The action of $W_{\mathbb{Q}}$ on $\mathrm{GL}_{2 m}(\mathbb{C})$ factors through $W_{\mathbb{Q}} \rightarrow \operatorname{Gal}(K / \mathbb{Q})$ and the non-trivial element of $\operatorname{Gal}(K / \mathbb{Q})$ acts by $g \mapsto g^{*}$, where

$$
\begin{aligned}
& g^{*}=\left(\begin{array}{cc}
0 & -w_{1}^{-1} \\
w_{1} & 0
\end{array}\right) \cdot{ }^{\mathrm{t}} g^{-1} \cdot\left(\begin{array}{cc}
0 & w_{1}^{-1} \\
-w_{1} & 0
\end{array}\right), \\
& w_{1}=\left(\begin{array}{c}
{ }^{\prime} \\
-1
\end{array}\right) \in \mathrm{GL}_{m}(\mathbb{C}) .
\end{aligned}
$$




\section{T. IKEDA}

The homomorphism st : ${ }^{L} \mathcal{G} \rightarrow \mathrm{GL}_{4 m}(\mathbb{C})$ is defined by

$$
g \rtimes u \mapsto \begin{cases}\left(\begin{array}{cc}
g & 0 \\
0 & g^{*}
\end{array}\right) & \text { if } u \in W_{K}, \\
\left(\begin{array}{cc}
0 & g \\
g^{*} & 0
\end{array}\right) & \text { otherwise. }\end{cases}
$$

Here, $W_{K}$ is the Weil group of $K$. Let $L(s, f, \chi)$ be the twist of $L(s, f)$ by $\chi$. In terms of the Euler product, $L(s, f, \chi)$ is defined by

$$
L(s, f, \chi)=\prod_{p \nmid D}\left(1-\alpha_{p} \chi(p) p^{k-s}\right)^{-1}\left(1-\beta_{p} \chi(p) p^{k-s}\right)^{-1} \prod_{q \mid D}\left(1-\alpha_{p}^{-1} p^{k-s}\right)^{-1}
$$

if $m$ is even, and by

$$
L(s, f, \chi)=\prod_{p \nmid D}\left(1-\alpha_{p} \chi(p) p^{k-s}\right)^{-1}\left(1-\beta_{p} \chi(p) p^{k-s}\right)^{-1}
$$

if $m$ is odd. As in [Ike01, $\S 11$ ], Theorem 13.6 implies the following theorem.

Theorem 18.1. Let $m, n$, and $f$ be as in Theorem 5.1 or as in Theorem 5.2. Assume that $\operatorname{Lift}^{(m)}(f) \neq 0$. Let $L\left(s\right.$, Lift ${ }^{(m)}(f)$, st) be the L-function of Lift ${ }^{(m)}(f)$ associated to st : ${ }^{L} \mathcal{G} \rightarrow$ $\mathrm{GL}_{4 m}(\mathbb{C})$. Then up to bad Euler factors, $L\left(s\right.$, Lift $^{(m)}(f)$, st $)$ is equal to

$$
\prod_{i=1}^{m} L\left(s+k+n-i+\frac{1}{2}, f\right) L\left(s+k+n-i+\frac{1}{2}, f, \chi\right) .
$$

In terms of the Arthur conjecture, Theorem 18.1 can be interpreted as follows. From now on, we assume the Arthur conjecture. Let $\mathcal{L}_{\mathbb{Q}}$ be the hypothetical Langlands group for $\mathbb{Q}$. The canonical homomorphism $\mathcal{L}_{\mathbb{Q}} \rightarrow W_{\mathbb{Q}}$ is denoted by pr. Let $\tau$ be an irreducible cuspidal automorphic representation of $\mathrm{GL}_{2}\left(\mathbb{A}_{\mathbb{Q}}\right)$ generated by $f$. Note that the central character $\omega_{\tau}$ is equal to $\chi^{m-1}$. We denote the Langlands parameter of $\tau$ by $\rho_{\tau}: \mathcal{L}_{\mathbb{Q}} \rightarrow \mathrm{GL}_{2}(\mathbb{C})$. We normalize the irreducible representation $\mathrm{Sym}^{m-1}: \mathrm{SL}_{2}(\mathbb{C}) \rightarrow \mathrm{SL}_{m}(\mathbb{C})$ so that

$$
{ }^{\mathrm{t}} \operatorname{Sym}^{m-1}(x)^{-1}=w_{1} \operatorname{Sym}^{m-1}(x) w_{1}^{-1}, \quad x \in \mathrm{SL}_{2}(\mathbb{C}) .
$$

We put

$$
\rho_{\tau}^{(m)}(u)=\left(\begin{array}{ll}
\omega_{\tau}(u) a \cdot \mathbf{1}_{m} & b \cdot \mathbf{1}_{m} \\
\omega_{\tau}(u) c \cdot \mathbf{1}_{m} & d \cdot \mathbf{1}_{m}
\end{array}\right) \rtimes \operatorname{pr}(u)
$$

for $u \in \mathcal{L}_{\mathbb{Q}}$,

$$
\left(\begin{array}{ll}
a & b \\
c & d
\end{array}\right)=\rho_{\tau}(u)
$$

and put

$$
\rho_{\tau}^{(m)}(x)=\left(\begin{array}{cc}
\operatorname{Sym}^{m-1}(x) & 0 \\
0 & \operatorname{Sym}^{m-1}(x)
\end{array}\right) \rtimes 1
$$

for $x \in \mathrm{SL}_{2}(\mathbb{C})$. Then we get a homomorphism $\rho_{\tau}^{(m)}: \mathcal{L}_{\mathbb{Q}} \times \mathrm{SL}_{2}(\mathbb{C}) \rightarrow{ }^{L} \mathcal{G}$. One can easily show that $L\left(s\right.$, st $\left.\circ \rho_{\tau}^{(m)}\right)=L\left(s\right.$, Lift $^{(m)}(f)$, st $)$. Thus the Arthur parameter of Lift ${ }^{(m)}(f)$ should be $\rho_{\tau}^{(m)}$.

In Case $\mathrm{O}, \tau$ can be considered as an automorphic representation of $\mathrm{PGL}_{2}(\mathbb{A})$. The automorphic representation generated by $\operatorname{Lift}^{(m)}(f)$ can be considered as a functorial lift of $\tau$ by the $L$ homomorphism

$$
{ }^{L} \mathrm{PGL}_{2} \times \mathrm{SL}_{2}(\mathbb{C})=\mathrm{SL}_{2}(\mathbb{C}) \times W_{\mathbb{Q}} \times \mathrm{SL}_{2}(\mathbb{C}) \rightarrow{ }^{L} \mathcal{G}
$$




\section{LIFTING OF HERMITIAN MODULAR FORMS}

given by

$$
\left(\begin{array}{ll}
a & b \\
c & d
\end{array}\right) \times u \times x \mapsto\left(\begin{array}{ll}
a \cdot \operatorname{Sym}^{m-1}(x) & b \cdot \operatorname{Sym}^{m-1}(x) \\
c \cdot \operatorname{Sym}^{m-1}(x) & d \cdot \operatorname{Sym}^{m-1}(x)
\end{array}\right) \rtimes u .
$$

In Case E, we take an auxiliary Hecke character $\underline{\hat{\chi}}: \mathbb{A}_{K}^{\times} / K^{\times} \rightarrow \mathbb{C}^{\times}$such that $\left.\underline{\hat{\chi}}\right|_{\mathbb{A}_{\mathbb{Q}}^{\times}}=\underline{\chi}$. Consider the algebraic groups $G^{\prime}$ and $Z$ defined over $\mathbb{Q}$ such that

$$
\begin{aligned}
G^{\prime}(\mathbb{Q}) & =\left\{(x, g) \in K^{\times} \times \mathrm{GL}_{2}(\mathbb{Q}) \mid \mathrm{N}_{K / \mathbb{Q}}(x) \operatorname{det} g=1\right\}, \\
Z(\mathbb{Q}) & =\left\{\left(z, z^{-1}\right) \in G^{\prime} \mid z \in \mathbb{Q}^{\times}\right\} \simeq \mathbb{Q}^{\times} .
\end{aligned}
$$

Then there is an exact sequence

$$
1 \rightarrow Z \rightarrow G^{\prime} \rightarrow \mathrm{U}(1,1) \rightarrow 1 .
$$

Here, the map $G^{\prime} \rightarrow \mathrm{U}(1,1)$ is given by $(x, g) \mapsto x g$. Then $\underline{\hat{\chi}}^{-1} \otimes \tau$ induces an automorphic representation $\hat{\tau}$ of $\mathrm{U}(1,1)(\mathbb{A})$. Fix an element $u_{0} \in W_{\mathbb{Q}}, u_{0} \notin W_{K}$. Then one can define an $L$ homomorphism

$$
{ }^{L} \mathrm{U}(1,1) \rtimes \mathrm{SL}_{2}(\mathbb{C})=\mathrm{GL}_{2}(\mathbb{C}) \rtimes W_{\mathbb{Q}} \times \mathrm{SL}_{2}(\mathbb{C}) \rightarrow{ }^{L} \mathcal{G}
$$

by

$$
\begin{aligned}
\left(\begin{array}{ll}
a & b \\
c & d
\end{array}\right) & \mapsto\left(\begin{array}{ll}
a \cdot \mathbf{1}_{m} & b \cdot \mathbf{1}_{m} \\
c \cdot \mathbf{1}_{m} & d \cdot \mathbf{1}_{m}
\end{array}\right) \rtimes 1, \quad\left(\begin{array}{ll}
a & b \\
c & d
\end{array}\right) \in \mathrm{GL}_{2}(\mathbb{C}), \\
u & \mapsto \underline{\hat{\chi}}(u) \cdot \mathbf{1}_{2 m} \rtimes u, \quad u \in W_{K}, \\
u_{0} & \mapsto\left(\begin{array}{cc}
-\mathbf{1}_{m} & 0 \\
0 & \mathbf{1}_{m}
\end{array}\right) \rtimes u_{0}, \\
x & \mapsto\left(\begin{array}{cc}
\operatorname{Sym}^{m-1}(x) & 0 \\
0 & \operatorname{Sym}^{m-1}(x)
\end{array}\right) \rtimes 1, \quad x \in \mathrm{SL}_{2}(\mathbb{C}) .
\end{aligned}
$$

The automorphic representation generated by $\operatorname{Lift}^{(m)}(f)$ can be considered as a functorial lift of $\hat{\tau}$ by this $L$-homomorphism.

We recall Arthur's conjectural multiplicity formula [Art89]. Let $\psi: \mathcal{L}_{\mathbb{Q}} \times \mathrm{SL}_{2}(\mathbb{C}) \rightarrow{ }^{L} G=$ $\hat{G} \rtimes W_{\mathbb{Q}}$ be an $A$-parameter for a quasi-split reductive algebraic group $G$. Let $\Pi(\psi)$ and $\Pi_{v}(\psi)$ be the global and local $A$-packet for $\psi$. Set $\mathcal{S}=\operatorname{Cent}_{\hat{G}}(\psi) / \operatorname{Cent}(\hat{G})^{W_{\mathbb{Q}}}$. The group $\mathcal{S}$ is closely related to the internal structure of the $A$-packet. Arthur conjectured that there exist a pairing $\left\langle s, \pi_{v}\right\rangle_{v}: \mathcal{S} \times \Pi_{v}(\psi) \rightarrow \mathbb{C}$ and a 'sign character' $\epsilon_{\psi}(s) \in\{ \pm 1\}$ for each $s \in \mathcal{S}$ and $\pi_{v} \in \Pi_{v}(\psi)$. (In fact, Arthur treated these objects locally.) For each $\pi=\bigotimes_{v}^{\prime} \pi_{v}, \pi_{v} \in \Pi_{v}(\psi)$, set

$$
m_{\psi}(\pi)=\frac{1}{\sharp \mathcal{S}} \sum_{s \in \mathcal{S}} \epsilon_{\psi}(s) \prod_{v}\left\langle s, \pi_{v}\right\rangle_{v} .
$$

Then Arthur's conjectural multiplicity formula says that the multiplicity of $\pi$ in the space of squareintegrable automorphic forms on $G(\mathbb{A})$ is equal to $\sum_{\pi \in \Pi(\psi)} m_{\psi}(\pi)$.

Now we consider the case $\psi=\rho_{\tau}^{(m)}$. In this case, the sign character $\varepsilon_{\psi}(s)$ must be trivial. One can easily show that

$$
\mathcal{S} \simeq \begin{cases}\{ \pm 1\} & \text { if } \tau \text { comes from a Hecke character of } K \\ \{1\} & \text { otherwise }\end{cases}
$$

Let $\pi=\bigotimes_{v} \pi_{v}$ be an element of the conjectural $A$-packet $\Pi\left(\rho_{\tau}^{(m)}\right)$. If $\mathcal{S}=\{1\}$, then the Arthur conjectural multiplicity formula suggests that any element of the global $A$-packet should be automorphic. In particular, Corollary 14.2 is compatible with the Arthur conjectural multiplicity formula. 


\section{T. IKEDA}

Now assume that $\mathcal{S} \simeq\{ \pm 1\}$. Note that $m$ must be even, since a normalized Hecke eigenform of $S_{2 k}\left(\mathrm{SL}_{2}(\mathbb{Z})\right)$ does not come from a Hecke character of a quadratic field. Let $s \in \mathcal{S}$ be the non-trivial element.

For each prime $p, \tau_{p}$ is a principal series induced from

$$
\left(\begin{array}{ll}
x & * \\
0 & y
\end{array}\right) \mapsto \mu_{p}(x) \nu_{p}(y)
$$

Here, $\mu_{p}$ and $\nu_{p}$ are characters of $\mathbb{Q}_{p}$ such that $\mu_{p}(x)=\alpha_{p}^{\text {ord }_{p} x}$ and $\mu_{p} \nu_{p}=\underline{\chi}_{p}$. The local $A$-packet $\Pi_{p}\left(\rho_{\tau_{p}}^{(m)}\right)$ should consist of the irreducible components of the degenerate principal series induced from the character

$$
\left(\mu_{p} \circ \mathrm{N}_{K_{p} / \mathbb{Q}_{p}} \circ \text { det }\right): P\left(\mathbb{Q}_{p}\right) \rightarrow \mathbb{C}^{\times} .
$$

Here, $P$ is the Siegel parabolic subgroup of $\mathcal{G}$. If $\pi_{p} \in \Pi_{p}\left(\rho_{\tau_{p}}^{(m)}\right)$ has a vector fixed by the maximal compact subgroup $\mathcal{G}\left(\mathbb{Q}_{p}\right) \cap \mathrm{GL}_{2 m}\left(\mathcal{O}_{p}\right)$, then the character $\left\langle *, \pi_{p}\right\rangle_{p}$ should be trivial.

At infinite place, the local $A$-packet should consist of certain cohomologically induced modules (see Adams and Johnson [AJ87]). If $\pi_{\infty}$ is the lowest weight module of $\mathcal{G}(\mathbb{R})$ generated by Lift ${ }^{(m)}(f)$, then $\left\langle s, \pi_{\infty}\right\rangle_{\infty}=(-1)^{m / 2}$ by the result of [AJ87]. Therefore Corollary 15.21 is compatible with the Arthur conjectural multiplicity formula.

Next, we describe the multiplicity formula for $\mathcal{G}_{1}=\mathrm{SU}(m, m)$. We consider only Case E. Let $\psi$ be the Arthur parameter $\mathcal{L}_{\mathbb{Q}} \times \mathrm{SL}_{2}(\mathbb{C}) \rightarrow{ }^{L} \mathcal{G}_{1}$ induced from $\rho_{\tau}^{(m)}$. In this case, the group $\mathcal{S}$ can be identified with the group

$$
\left\{\chi_{Q} \mid f_{Q}=f\right\} \text {. }
$$

For a prime $p$, the local $A$-packet $\Pi_{p}(\psi)$ should consist of the irreducible constituents of the degenerate principal series induced from the character

$$
\left(\mu_{p}^{2} \circ \text { det }\right): P_{1}\left(\mathbb{Q}_{p}\right) \rightarrow \mathbb{C}^{\times}
$$

Here, $P_{1}$ is the Siegel parabolic subgroup of $\mathcal{G}_{1}$. We denote the maximal compact subgroup $\operatorname{GL}_{2 m}\left(\mathcal{O}_{p}\right)$ $\cap \mathcal{G}_{1}\left(\mathbb{Q}_{p}\right)$ by $\mathcal{K}_{1, p}$. If $\pi_{p}$ is the element of the packet $\Pi_{p}(\psi)$ with non-trivial $\mathcal{K}_{1, p}$-fixed vector, then the pairing $\left\langle *, \pi_{p}\right\rangle_{p}$ should be trivial. Let $\mathfrak{c}$ be an integral ideal of $K$ such that $C=\mathrm{N}(\mathfrak{c})$ is prime to $D_{K}$. If $\pi_{p} \in \Pi_{p}(\psi)$ has a $\mathcal{K}_{1}[\mathfrak{c}]_{p}$-fixed vector, where $\mathcal{K}_{1}[\mathfrak{c}]_{p}$ is the closure of $\Gamma_{K}^{(m)}[\mathfrak{c}] \cap \mathcal{G}_{1}(\mathbb{Q})$ in $\mathcal{G}_{1}\left(\mathbb{Q}_{p}\right)$, then the pairing $\mathcal{S} \times \Pi_{p}\left(\rho_{\tau}^{(m)}\right)$ should be given by

$$
\left\langle\chi_{Q}, \pi_{p}\right\rangle_{p}=\chi_{Q}(p)^{\operatorname{ord}_{p} C}, \quad p \nmid D_{K} .
$$

For $v=\infty$, we have $\left\langle\chi_{Q}, \pi_{\infty}\right\rangle_{\infty}=\chi_{Q}(-1)^{m / 2}$. Set $\pi=\bigotimes_{p<\infty} \pi_{p} \otimes \pi_{\infty}$, where $\pi_{p} \in \Pi_{p}(\psi)$ has a non-trivial $\mathcal{K}_{1}[\mathfrak{c}]_{p}$-fixed vector. Then $m_{\psi}(\pi)=1$ if and only if $\varepsilon(Q)=\chi_{Q}(-1)^{m / 2}$ for any $Q \subset Q_{D}$ such that $f_{Q}=f$. Therefore Corollary 15.19 is compatible with the Arthur conjectural multiplicity formula.

\section{ACKNOWLEDGEMENTS}

The author thanks Professors Ibukiyama, Nagaoka and Sugano for useful discussions. Professor Ichino kindly helped to carry out the calculation of the Petersson norm of the Maass lift. The author thanks Professor Hiraga for careful reading of the earlier version of the manuscript. The author also thanks the referee for his suggestions. 


\section{LIFTING OF HERMITIAN MODULAR FORMS}

\section{REFERENCES}

AJ87 J. Adams and J. Johnson, Endoscopic groups and packets of non-tempered representations, Compositio Math. 64 (1987), 271-309.

Art89 J. Arthur, Unipotent automorphic representations: conjectures, Astérisque 171-172 (1989), $13-71$.

Bra51 H. Braun, Hermitian modular functions. III, Ann. of Math. (2) 53 (1951), 143-160.

EZ85 M. Eichler and D. Zagier, The theory of Jacobi forms, Progress in Mathematics, vol. 55 (Birkhäuser, Boston, MA, 1985).

Gri90 V. A. Gritsenko, The Maass space for $\mathrm{SU}(2,2)$. The Hecke ring, and zeta functions (Russian), Translated in Proc. Steklov Inst. Math. 1991, no. 4, 75-86. Galois theory, rings, algebraic groups and their applications (Russian), Trudy Mat. Inst. Steklov. 183 (1990), 68-78, 223-225.

Hec81 E. Hecke, Lectures on the theory of algebraic numbers, Translated from the German, Graduate Texts in Mathematics, vol. 77 (Springer, Berlin, 1981).

Hid00 H. Hida, Modular forms and Galois cohomology (Cambridge University Press, Cambridge, 2000).

Ike94 T. Ikeda, On the theory of Jacobi forms and the Fourier-Jacobi coefficients of Eisenstein series, J. Math. Kyoto Univ. 34 (1994), 615-636.

Ike01 T. Ikeda, On the lifting of elliptic cusp forms to Siegel cusp forms of degree $2 n$, Ann. of Math. (2) 154 (2001), 641-681.

Ike06 T. Ikeda, Pullback of the lifting of elliptic cusp forms and Miyawaki's conjecture, Duke Math. J. 131 (2006), 469-497.

Kli56 H. Klingen, Über die Erzeugenden gewisser Modulgruppen, Nachr. Akad. Wiss. Goettingen. Math.Phys. Kl. IIa, 1956 (1956), 173-185.

Klo07a K. Klosin, Congruences among modular forms on $\mathrm{U}(2,2)$ and the Bloch-Kato conjecture, Ann. Inst. Fourier (Grenoble), to appear.

Klo07b K. Klosin, Adelic Maass spaces on U(2,2), Preprint, arXiv:0706.2828v1.

Koh02 W. Kohnen, Lifting modular forms of half-integral weight to Siegel modular forms of even genus, Math. Ann. 322 (2002), 787-809.

Koj82 H. Kojima, An arithmetic of Hermitian modular forms of degree two, Invent. Math. 69 (1982), $217-227$.

Kri91 A. Krieg, The Maass spaces on the Hermitian half-space of degree 2, Math. Ann. 289 (1991), 663-681.

KS97 S. Kudla and W. J. Sweet Jr., Degenerate principal series representations for U $(n, n)$, Israel J. Math. 98 (1997), 253-306.

LL79 J.-P. Labesse and R. P. Langlands, L-indistinguishability for SL(2), Canad. J. Math. 31 (1979), 726-785.

Miy89 T. Miyake, Modular forms (Springer, Berlin, 1989).

Nag92 S. Nagaoka, On Eisenstein series for the Hermitian modular groups and the Jacobi groups, Abh. Math. Sem. Univ. Hamburg 62 (1992), 117-146.

RS91 S. Raghavan and J. Sengupta, A Dirichlet series for Hermitian modular forms of degree 2, Acta Arith. 58 (1991), 181-201.

Sch85 W. Scharlau, Quadratic and Hermitian forms, Grundlehren der Mathematischen Wissenschaften, vol. 270 (Springer, Berlin, 1985).

Shi97 G. Shimura, Euler products and Eisenstein series, CBMS Regional Conference Series in Mathematics, vol. 93 (American Mathematical Society, Providence, RI, 1997).

Shi75 T. Shintani, On constriction of holomorphic cusp forms of half integral weight, Nagoya Math. J. 58 (1975), 83-126.

Sug85 T. Sugano, On Maass space for $\mathrm{SU}(2,2)$ (Japanese), Surikaisekikenkyusho Kokyuroku (RIMS Kokyuroku) 546 (1985) 1-16.

Sug95 T. Sugano, Jacobi forms and the theta lifting Comment. Math. Univ. St. Pauli 44 (1995), 1-58. 


\section{LIFTING OF HERMITIAN MODULAR FORMS}

Tat79 J. Tate, Number theoretic background, Proceedings of Symposia in Pure Mathematics, vol. XXXIII, part 2 (American Mathematical Society, Providence, RI, 1979), 3-26.

Tamotsu Ikeda ikeda@math.kyoto-u.ac.jp

Graduate school of Mathematics, Kyoto University, Kitashirakawa, Kyoto, 606-8502, Japan 